\title{
Use of Ceramic Materials in Waste-Package Systems for Geologic Disposal of Nuclear Wastes
}

\author{
H. T. Fullam
}

December 1980

Prepared for the U.S. Department of Energy under Contract DE-AC06-76RLO 1830

Pacific Northwest Laboratory Operated for the U.S. Department of Energy by Battelle Memorial Institute 
NOTICE

This report was prepared as an account of worksponsored by the United States Government. Neither the United States nor the Department of Energy, nor any of their employees, nor any of their contractors, subcontractors, or their employees, makes any warranty, express or implied, or assumes any legal liability or responsibility for the accuracy completeness or usefulness of any information. apparatus, product or process disclosed, or represents that its use would not infringe privately owned rights.

The views, opinions and conclusions contained in this report are those of the contractor and do not necessarily represent those of the United States Government or the United States Department af Energy.

PACIFIC NORTHWEST LABORATORY

operated by

BATTELLE

for the

UNITED STATES DEPARTMENT OF ENERGY

Under Contract DE-AC06-76RLO 1830

\author{
Printed in the United States of America \\ Availabie trom \\ National Technical Intormation Service \\ United States Department of Commerct \\ 5285 Port Roval Rouid \\ Springtield. Virginia 22151
}

Price: Printed Copy 5

$\therefore$ Microficne 53.00

NTIS

-Pages - Selling Price

$001.025 \quad \$ 400$

026-050 $\quad 54.50$

$057.075 \quad \$ 5.25$

076. $100 \quad \$ 6.00$

$107.125 \quad \$ 6.50$

$126-150 \quad \$ 77.25$

$151-175 \quad \$ 8.00$

$176-200 \quad 59.00$

$201.225 \quad 59.25$

$226-250 \quad 59.50$

$251-275-\quad 510.75$

276-300 $\quad 511.00$ 


\section{7}

USE OF CERAMIC MATERIALS IN WASTE-PACKAGE SYSTEMS FOR GEOLOGIC DISPOSAL OF NUCLEAR WASTES

H. T. Fullam

December 1980

Prepared for the U.S. Department of Energy under Contract DE-AC06-76RLO 1830

Pacific Northwest Laboratory

Richland, Washington 99352 

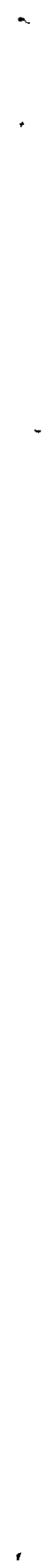

.

, 
A program is currently under way at $P$ acific Northwest Laboratory to develop durable, long-lived packages for the containment of nuclear wastes under conditions of deep geologic disposal. One phase of the program consists of a study to investigate the potential use of ceramic materials as components in the waste package systems. The initial objective of the study was to screen and compare a large number of ceramic materials and identify the best materials for the proposed application. The principal method used to screen the candidates was to subject samples of each material to a series of leaching tests and to determine their relative resistance to attack by the leach solutions.

A total of 14 ceramic materials, plus graphite and basalt were evaluated using three different leach solutions: demineralized water, a synthetic Hanford ground water, and a synthetic WIPP brine solution. The ceramic materials screened were $\mathrm{Al}_{2} \mathrm{O}_{3}(99 \%), \mathrm{Al}_{2} \mathrm{O}_{3}$ (99.8\%), mullite $\left(2 \mathrm{Al}_{2} \mathrm{O}_{3} \cdot \mathrm{SiO}_{2}\right)$, vitreous silica $\left(\mathrm{SiO}_{2}\right), \mathrm{BaTiO}_{3}, \mathrm{CaTiO}_{3}, \mathrm{CaTiSiO}_{5}, \mathrm{TiO}_{2}, \mathrm{ZrO}_{2}, \mathrm{ZrSiO} \mathrm{C}_{4}$, Pyroceram 9617, ${ }^{\circledR}$ and Marcor ${ }^{\circledR}$ Code 9658 machinable glass-ceramic. Five leaching tests were used to screen the candidates:

- a static leach test at $100^{\circ} \mathrm{C}$,

- a hydrothermal static leach test at $150^{\circ} \mathrm{C}$,

- a hydrothermal static leach test at $250^{\circ} \mathrm{C}$,

- a dynamic (flow) leach test at $90^{\circ} \mathrm{C}$, and

- a dynamic (flow) leach test at $250^{\circ} \mathrm{C}$.

Average leach rates for the materials tested were determined from analyses of the leach solutions and/or sample weight loss measurements.

Averaged leach rate measurements provide one method of screening the candidates, but the results obtained can be misleading because they provide no information on the extent to which localized attack or alteration reactions may occur with a given material. Therefore, additional analysis of the specimens is needed after testing, such as ceramographic examination and scanning electron microscopy. Because of the limited scope of the present study, evaluation of the specimens was limited to ceramographic examination.

Whenever possible the materials tested were obtained as dense, impervious shapes from commercial sources. Test specimens were cut from the commercial shapes using a diamond saw. Some material could only be obtained in powder form. These materials were compacted into dense pellets at PNL by hot-pressing or cold-pressing and sintering.

Based on an overall evaluation of the leach rate data, five of the materials tested-namely graphite, $\mathrm{TiO}_{2}, \mathrm{ZrO}_{2}$, and the two grades of alumina--exhibited much greater resistance to leaching than did the other materials tested. Some of the other materials exhibited good leach resistance in one or two tests, but on an overall basis they were inferior to the five

${ }^{\circledR}$ Corning Glass Works, Corning, New York 
materials mentioned above. In addition, most of the materials having poor leach resistance were also susceptible to alteration reactions (especially at $250^{\circ} \mathrm{C}$ ), which was not a problem with the more leach-resistant materials.

Based on all the experimental data obtained, and considering other factors such as cost, availability, fabrication technology, and mechanical and physical properties, graphite and alumina are the preferred candidates for the barrier application. The secondary choices are $\mathrm{TiO}_{2}$ and $\mathrm{ZrO}_{2}$. 
SUMMARY

FIGURES

TABLES

1.0 INTRODUCTION

2.0 OBJECTIVES

3.0 EXPERIMENTAL

3.1 TESTING CRITERIA

3.2 TEST PROCEDURES

3.3 MATERIALS EVALUATED

3.3.1 Material Selection

3.3.2 Sample Preparation

3.4 LEACH SOLUTIONS

3.5 ANALYTICAL PROCEDURES

4.0 RESULTS AND DISCUSSION

4.1 LEACH RATES

4.1.1 Static Leach Tests at $100^{\circ} \mathrm{C}$

viji

4.1.2 Dynamic Leach Tests at $90^{\circ} \mathrm{C}$

4.1.3 Dynamic Leach Tests at $250^{\circ} \mathrm{C}$

4.1.4 Static Leach Tests at $150^{\circ} \mathrm{C}$

4.1.5 Static Leach Tests at $250^{\circ} \mathrm{C}$

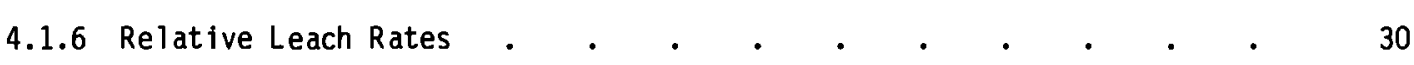

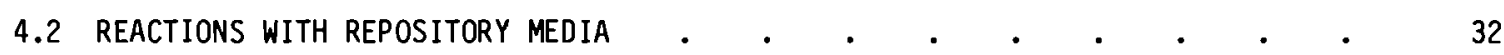

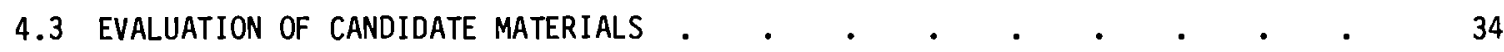

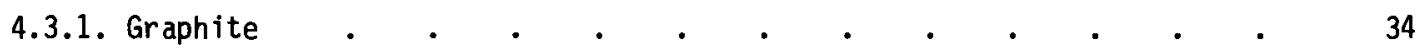

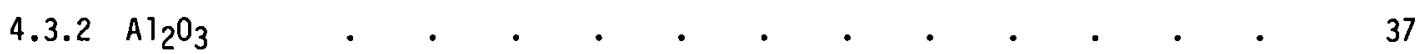

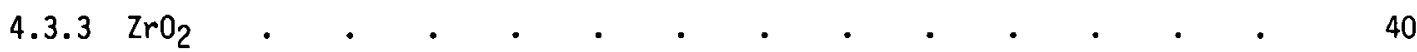

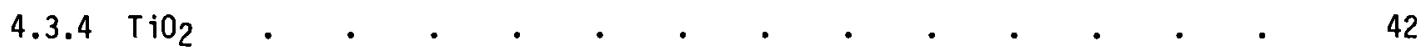

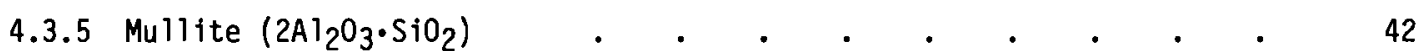

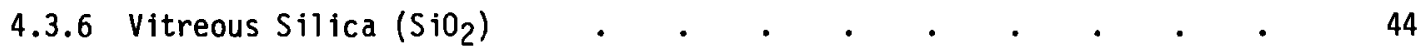

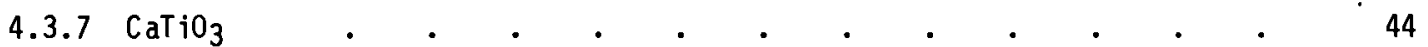




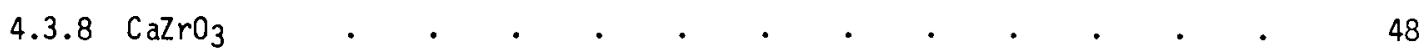

$4.3 .9 \mathrm{CaTiSiO}_{5} \quad . \quad . \quad . \quad . \quad . \quad . \quad . \quad . \quad . \quad . \quad . \quad . \quad . \quad 48$

4.3 .10 Basalt . . . . . . . . . . . . . . . 51

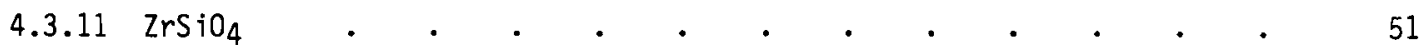

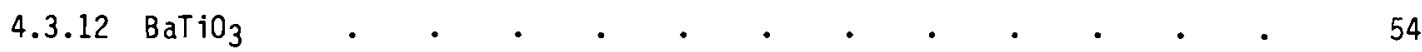

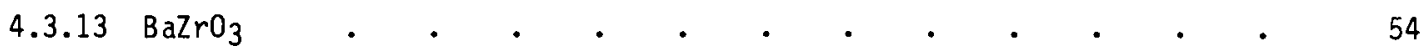

4.3.14 Pyroceram 9617 .

4.3.15 Marcor 9658 . . . . . . . . . . . . . . . . . . 57

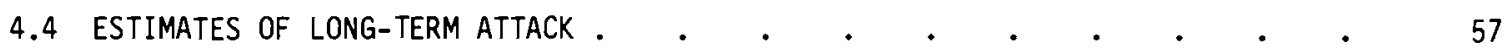

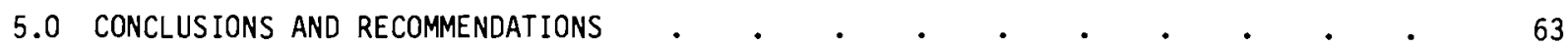

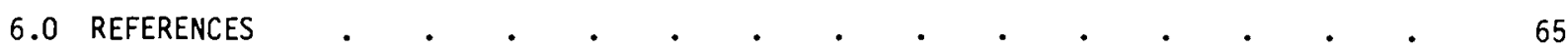

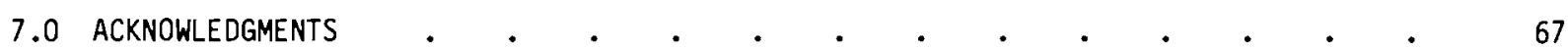

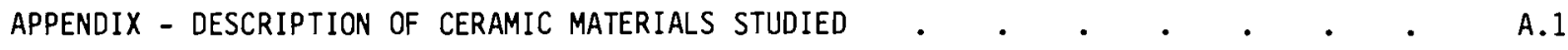

$\mathrm{Al}_{2} \mathrm{O}_{3}(99 \%) \quad$.

$\mathrm{Al}_{2} \mathrm{O}_{3}(99.8 \%) \quad$.

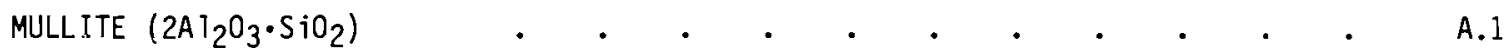

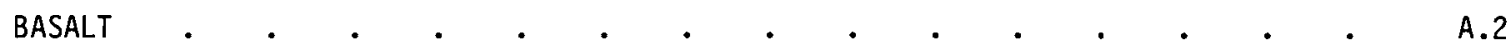

$\mathrm{BaTiO}_{3}$ •

$\mathrm{BaZrO}_{3} \cdot{ }^{\circ}$.

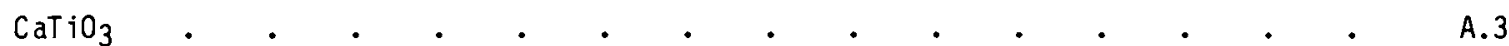

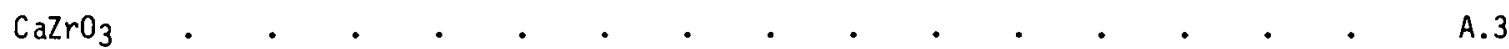

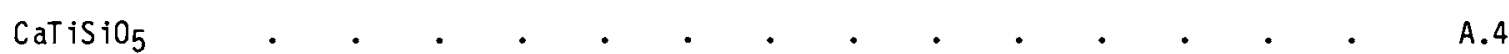

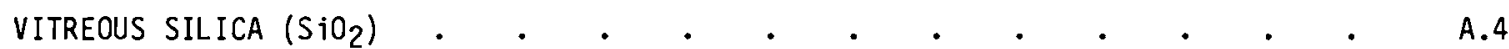

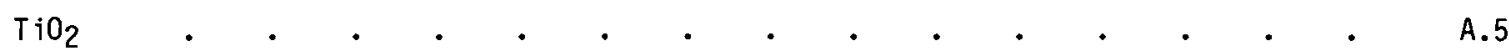

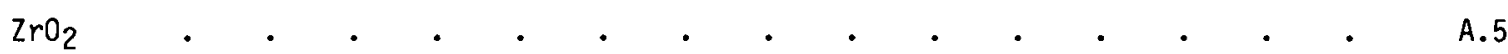

$\mathrm{ZrSiO}_{4}(\mathrm{ZIRCON}) \quad$.

GRAPHITE (TOYO)

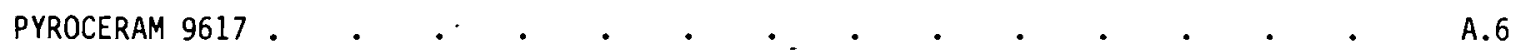

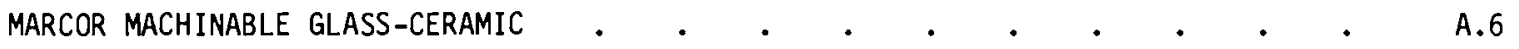




\section{FIGURES}

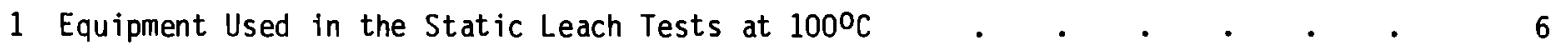

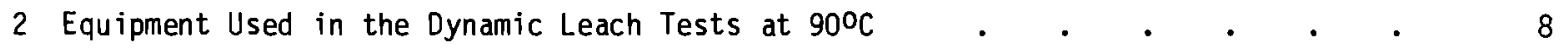

3 Photomicrographs of Graphite Specimens Before and After Testing . . . . 36

$4 \mathrm{Al}_{2} \mathrm{O}_{3}$ (99\%) Specimens Before and After Leaching . . . . . . . . 38

$5 \mathrm{Al}_{2} \mathrm{O}_{3}$ (99.8\%) Specimens Before and After Leaching . . . . . . . . . 39

$6 \mathrm{ZrO}_{2}$ Specimens Before and After Leaching . . . . . . . . . . . 41

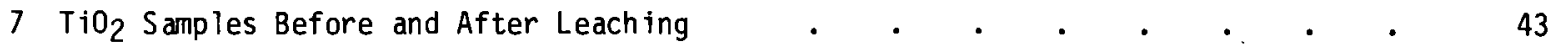

8 Micrographs of Mullite Test Specimens . . . . . . . . . . . . . $\quad$. 45

9 Micrographs of Vitreous Stlica Specimens Before and After Leaching . . . 46

$10 \mathrm{CaTiO}_{3}$ Specimens Before and After Leaching . . . . . . . . . . . . 47

$11 \mathrm{CaZrO}_{3}$ Specimens Before and After Leaching . . . . . . . . . . 49

12 Micrographs of $\mathrm{CaTiSiO}_{5}$ Specimens Before and After Leaching . . . . 50

13 Basalt Specimens Before and After Testing . . . . . . . . . . . 52

14 Photomicrographs of $\mathrm{ZrSiO}_{4}$ Specimens . . . . . . . . . . . . 53

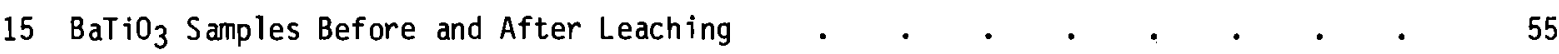

16 Micrographs of $\mathrm{BaZrO}_{3}$ Specimens Before and After Leaching . . . . . . 56

17 Micrographs of Pyroceram 9617 Specimens . . . . . . . . . . . . 58

18 Marcor Specimens Before and After Testing . . . . . . . . . . 59

19 The Effect of Temperature on the Leaching of Various Materials . . . . . 64 


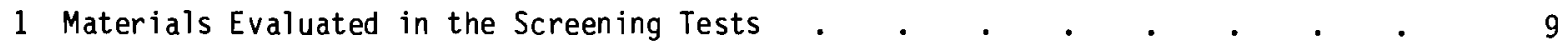

2 Composition of Solutions Used in the Leaching Experiments . . . • . $\quad$ • 11

3 Detection Limits for Cations Using the Induction-Coupled Plasma

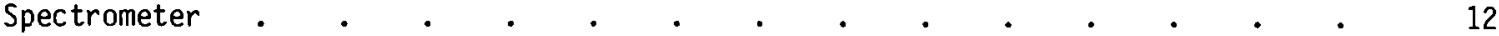

\begin{tabular}{l}
4 Average Leach Rates for Ceramic Materials in Demineralized Water at $100^{\circ} \mathrm{C}-{ }_{-}$ \\
Vented Static System \\
\hline
\end{tabular}

5 Leaching of Pyrex Glassware in Demineralized Water at $100^{\circ} \mathrm{C} \quad$ • $\quad$ • $\quad$. 17

6 Leaching of Ceramic Materials in Flowing Demineralized Water at $90^{\circ} \mathrm{C}$. $\quad . \quad 18$

7 Average Leach Rates for Ceramic Materials Exposed to Flowing Demineralized

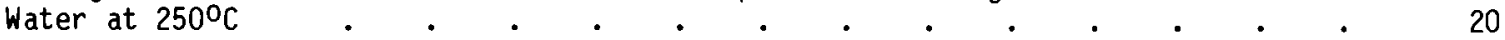

8 Extent of Localized Attack or Alteration Reactions in Ceramic Materials Exposed to Flowing Demineralized Water at $250^{\circ} \mathrm{C}$..$\quad$. . . . . . 22

9 Leaching of Ceramic Materials in Demineralized Water at $150^{\circ} \mathrm{C}$--Static

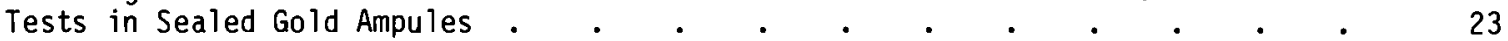

10 Leaching of Ceramic Materials in Hanford Ground Water at $150^{\circ} \mathrm{C}$--Static

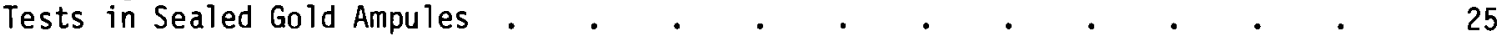

11 Leaching of Ceramic Materials in Brine at $150^{\circ} \mathrm{C}--$ Static Tests in Sealed

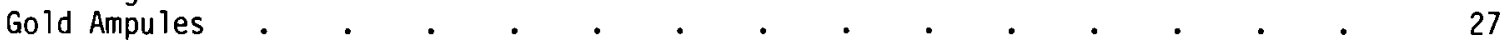

12 Leaching of Ceramic Materials in Demineralized Water at $250^{\circ} \mathrm{C}--\mathrm{Static}$

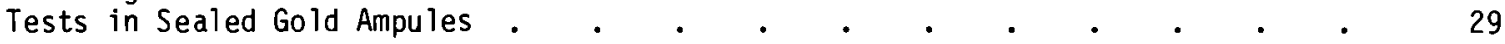

13 Leaching of Ceramic Materials in Hanford Ground Water at $250^{\circ} \mathrm{C}--\mathrm{Stat}$ ic Test in Sealed Gold Ampules .

14 Leaching of Ceramic Materials in Brine at $250^{\circ} \mathrm{C}--$ Static Tests in Sealed

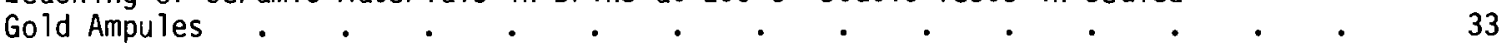

15 Relative Resistance of Ceramic Materials to Leaching Under Various Test Conditions (in Order of Decreasing Resistance) . . . . . . . . . . $\quad$. 35

16 Estimated Worst-Case Corrosion of Ceramic Material Exposed to Flowing Demineralized Water at $250^{\circ} \mathrm{C}$

17 Corrosion Rates of Metals and Alloys in Deoxygenated Solutions

\section{1} b 15 7 8 35 


\subsection{INTRODUCTION}

A study is under way at Pacific Northwest Laboratory (PNL) to develop long-lived wastepackage systems that can be used in the geologic disposal of nuclear wastes. The waste-package barrier system study represents one phase of PNL's High-Level Waste Immobilization Program, which is funded by the Department of Energy's Division of Waste Products.

The primary objective of the current study is to develop a waste-package engineered-barrier system that would serve to isolate the nuclear waste from the geologic repository environment for a period of 1000 years. The waste could be in the form of spent fuel or solidified high-level waste from a fuel reprocessing plant (i.e., glass, supercalcine, etc.). The 1000year period would ensure that before the waste came in contact with the geologic medium the heat-producing, short-lived radionuclides in the waste would have decayed to very low levels, and that the heat output would have decreased accordingly.

A waste package is currently defined as 1) the waste form, 2) the canister surrounding the waste form, 3) any canister overpack, 4) the hole sleeve, if any, and 5) the backfill medium. The term "engineered barrier" generally refers to any of the foregoing except for the waste form. A number of engineered-barrier system options are available. The simplest system would consist of the waste canister and the backfill material used to fill the hole in which the canister is placed. Additional barrier elements, such as an overpack or a hole sleeve, can be added to increase the reliability and integrity of the system. Ceramic materials are being considered for possible use as elements in the engineered-barrier systems. This report summarizes the results of some initial scouting experiments that were carried out to screen and compare a large number of ceramic materials and identify the best materials for the barrier application. The screening tests were not intended to demonstrate the usability of the ceramic materials in engineered-barrier systems, but simply to identify those ceramic materials that provide the best chance of developing acceptable barrier elements. Additional experimental work will be required to demonstrate that ceramic materials can be successfully used in engineered-barrier systems.

In addition to ceramics, metallic and polymeric materials are being investigated at PNL for potential use in engineered-barrier systems. 



\subsection{OBJECTIVES}

The primary objective of the ceramic materials investigation is to determine if practical ceramic-barrier elements can be developed that will provide protection of the primary waste canister for 1000 years under the conditions that would be encountered in a geologic repository. The current study is divided into two phases. The first phase consists of initial scouting studies whose basic objective is to screen and compare a large number of candidate ceramic materials and to select the best materials for detailed evaluation. The second phase is to carry out detailed experimental evaluation of the materials identified in Phase 1 and to determine if practical ceramic barrier elements can be developed. Continuation with Phase 2 of the study depends on the results obtained in Phase 1 and on the results obtained in the investigation of barriers made of other materials--i.e., metals and polymers. 



\subsection{EXPERIMENTAL}

Screening of the candidate ceramic materials was carried out through a series of leaching experiments conducted under various temperature and pressure conditions. Solid-solid reactions between the candidate materials and repository media were studied to a limited degree. Initial comparisons were based on experimentally determined leach rates and observed interactions. Other factors, such as availability, fabricability, mechanical and physical properties, and cost were considered in the final comparison and in selecting the best materials for detailed evaluation.

\subsection{TESTING CRITERIA}

Criteria and design parameters for geologic waste repositories have not been defined. Geologic media still under consideration for use as a repository include bedded salt, salt domes, granite, basalt, shale and volcanic tuffs. Various repository design considerations such as the age of the waste at the time of disposal, heat-loading, temperature limitations, backfill materials, etc., remain unclear. In this study it was impossible to consider all geologic alternatives and design parameters that enter into the construction of an engineeredbarrier system and waste repository. In order to reduce the degree of experimental testing to an acceptable level, it was necessary to make a number of assumptions in designing the test matrix:

- Only two repository types were considered: 1) bedded salt of the type found at the Waste Isolation Pilot Plant (WIPP) site in New Mexico, and 2) basalt typical of that found at Hanford.

- Aqueous solutions used in the leach tests were limited to: 1) demineralized water, 2) salt brine similar to that found at the WIPP site, and 3) a typical Hanford ground water.

- The maximum temperature for the hydrothermal leach tests, corresponding to a "wet" repository, was limited to $250^{\circ} \mathrm{C}$.

- The temperature in a "dry" repository would not exceed $375^{\circ} \mathrm{C}$.

- The possible effects of backfill materials on the reactions between the ceramic materials and repository environs was not considered.

- The effects of the radiation field on the reactions between the ceramic materials and repository environs was not considered.

\subsection{TEST PROCEDURES}

The ceramic materials to be screened were subjected to five different leach tests:

1. a static leach test at $100^{\circ} \mathrm{C}$

2. a static leach test at $150^{\circ} \mathrm{C}$

3. a static leach test at $250^{\circ} \mathrm{C}$ 
4. a dynamic (flow) leach test at $90^{\circ} \mathrm{C}$

5. a dynamic (flow) leach test at $250^{\circ} \mathrm{C}$.

In addition, the direct reaction of the ceramic material with the repository media was studied at $375^{\circ} \mathrm{C}$. Each of the test procedures is described in the following paragraphs.

Figure 1 shows the equipment used in carrying out the static leach test at $100^{\circ} \mathrm{C}$. The ceramic test specimen was measured to determine its geometric surface area and then weighed on a calibrated semi-microbalance (accuracy $= \pm 0.01 \mathrm{mg}$ ). The weighed specimen was placed in a one-liter Pyrex flat-bottom flask and a known volume of demineralized water was added. The ratio of the geometric surface area of the test specimen to water volume (S/V) was maintained constant at $0.02 \mathrm{~cm}^{-1}$ for all of the tests. A water-cooled Pyrex condenser was connected to the Pyrex flask, and a short section of Pyrex capillary tubing was placed on the outlet of the condenser. The water in the flask was heated to boiling on a hot plate and held at boiling for up to 49 days. Samples of the solution were taken at periodic intervals for analysis. The test specimens were also removed from the flask periodically, vacuum-dried, and weighed to monitor the weight change. The solution samples were collected through a 0.3-um Millipore filter using a polypropylene syringe and filter holder. The filter holder was placed in the solution and brought to the solution temperature before the sample was taken. As the solution entered the syringe, it was acidified to $2 \underline{\mathrm{N}^{+}}$with triply distilled nitric acid. The

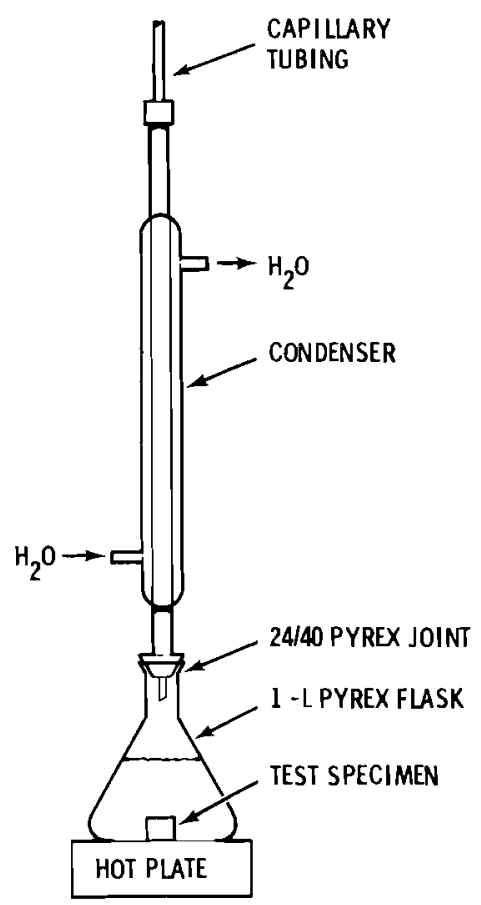

FIGURE 1. Equipment Used in the Static Leach Test at $100^{\circ} \mathrm{C}$ 
condenser and its capillary outlet reduced evaporation of water from the flask, but some water had to be added periodically to maintain the level. Blank tests, without ceramic specimens present, were also run in order to determine how much leaching of the Pyrex glassware occurred during testing. It is estimated that leach rates as low as $2 \times 10^{-8} \mathrm{~g} / \mathrm{cm}^{2}-\mathrm{d}$ could be measured by this procedure.

The static leach tests at $150^{\circ}$ and $250^{\circ} \mathrm{C}$ were carried out in sealed gold ampules. The following procedure was used for the tests. A weighed ceramic specimen of known geometric surface area was placed in a gold ampule, and a known volume of the leach solution was added. The gold ampule was then sealed by welding. The sealed ampule was placed in an autoclave filled with demineralized water. The autoclave was heated to $150^{\circ}$ or $250^{\circ} \mathrm{C}$, held at temperature for the required time, cooled, and the ampule was then removed and opened. The test specimen was removed from the ampule, washed, vacuum-dried, and weighed. The solution in the ampule was acidified with $2 \mathrm{M}$ high-purity hydrochloric acid and allowed to stand for several hours. After the solution was transferred to a sample bottle, the ampule was washed with more $2 M$ hydrochloric acid. The original solution and wash solution were combined and analyzed for dissolved species. The test was then repeated for a different time period using the same test specimen and fresh leach solution. Each specimen was tested for four time periods (1 day, 7 days, 14 days, and 21 days) using fresh leach solution for each test. The tests were carried out using the three leach solutions described in Section 3.4. An attempt was made to use the same $S / V$ ratio of $0.4 \mathrm{~cm}^{-1}$ (based on the specimens' geometric surface area) in all of the tests, but some minor variations in the ratio were inevitable with the different ceramic materials. The gold ampules used in the tests had an inner diameter of 0.50 in., and could hold up to $15 \mathrm{ml}$ of leach solution. After the final test, the ceramic specimen was subjected to ceramographic examination. It is estimated that leach rates as low as $5 \times 10^{-8} \mathrm{~g} / \mathrm{cm}^{2}-\mathrm{d}$ could be measured with the procedure.

The dynamic (flow) leach tests at $90^{\circ} \mathrm{C}$ were carried out using the equipment shown in Figure 2. The weighed test specimen, of known geometric surface area, was placed in a Teflon flask. The flask was placed in a water bath held at $90^{\circ} \mathrm{C}$. Demineralized water was pumped through the flask at a controlled rate using a peristaltic metering pump. The solution exiting the flask was filtered through an in-line 0.3-um Millipore filter and collected in a flask, which was also contained in the water bath. The average water-flow rate was approximately $0.2 \mathrm{ml} / \mathrm{min}$, and the residence time in the flask was about 20 hours. Samples of the solution in the collection flask were taken on a periodic basis and analyzed for dissolved species. The samples were taken using the syringe-Millipore filter technique described earlier. The ceramic specimen was also removed from the flasks at specific intervals, vacuum-dried, and weighed to monitor weight change as a function of exposure time. A multichannel peristaltic pump was used for the experiments, which allowed as many as ten tests to be carried out at one time. Leach rates as low as $1 \times 10^{-7} \mathrm{~g} / \mathrm{cm}^{2}-\mathrm{d}$ could be measured. 


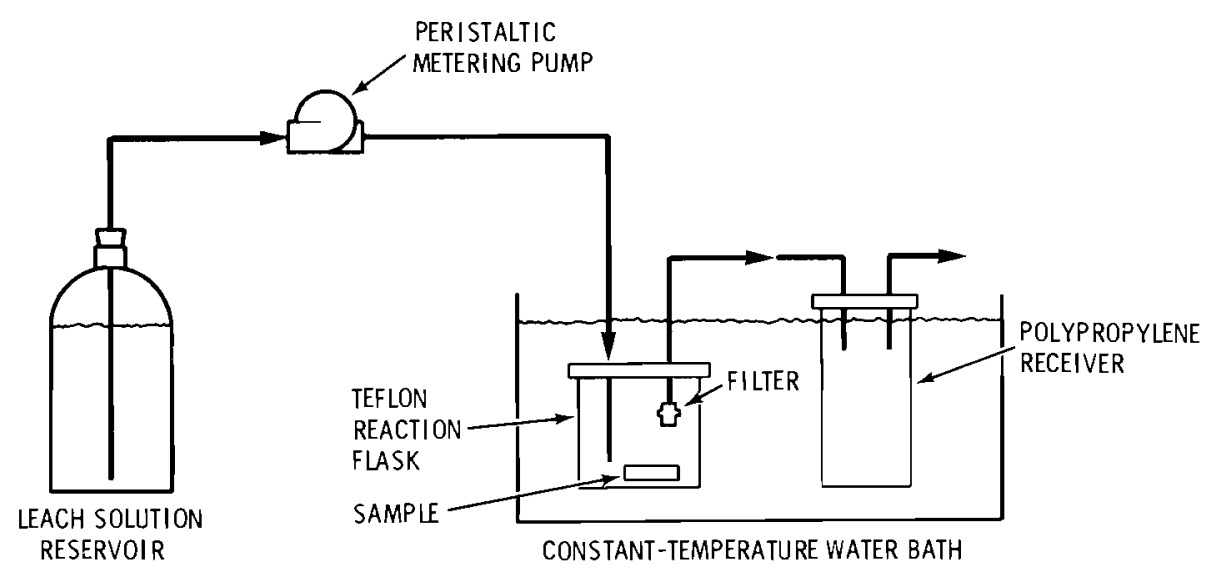

FIGURE 2. Equipment Used in the Dynamic Leach Tests at $90^{\circ} \mathrm{C}$

The dynamic (flow) leach tests at $250^{\circ} \mathrm{C}$ were carried out in demineralized water within a Hastelloy $c^{\circledR}$ autoclave. The weighed ceramic specimens, of known geometric area, were placed in the autoclave. Demineralized water was pumped through the autoclave at a controlled rate while the temperature was raised to $250^{\circ} \mathrm{C}$ and held at the temperature for the duration of the run. At specific intervals the autoclave was cooled and opened, and the ceramic specimens were removed, vacuum-dried and weighed. The specimens were then returned to the autoclave for additional testing. Each specimen was exposed for intervals of 1 day, 7 days, 14 days, and 21 or 23 days--giving a total exposure of 43 or 45 days. After the conclusion of the tests, the specimens were subjected to ceramographic examination. Eight ceramic materials were tested at one time and no attempt was made to analyze the demineralized water leaving the autoclave. The volume of the autoclave was $\sim 6.5 \mathrm{~L}$ and the water-flow rate was about $1.9 \mathrm{~L} / \mathrm{h}, \mathrm{giving}$ a residence time of approximateTy 3.4 hours. Leach rates as $10 \mathrm{w}$ as $2 \times 10^{-8} \mathrm{~g} / \mathrm{cm}^{2}-\mathrm{d} \mathrm{could}$ be measured. The scope of the study did not permit each material to be tested individually in the autoclave. The possibility exists that interactions between each group of materials tested could affect the results obtained, but these effects should be minimal considering the high water-flow rate and the low leach rates of the materials tested.

In addition to the leaching tests, the ceramic materials were subjected to one additional test. This test was intended to determine if any reaction might occur between a ceramic material and the repository medium--either basalt or salt. In these tests a weighed ceramic specimen was packed in crushed basalt or salt within a silica ampule. A small amount of water sufficient to give a partial pressure of $4 \mathrm{~mm} \mathrm{Hg}$ (STP) was added to the mixture and the ampule was sealed. The ampule was then heated to $375^{\circ} \mathrm{C}$ in a muffle furnace and held at temperature for the required time. At the conclusion of the test the ampule was cooled and opened. The test specimen was visually examined for possible reaction with the basalt or salt and then was weighed. The tests lasted 2200 and 4500 hours.

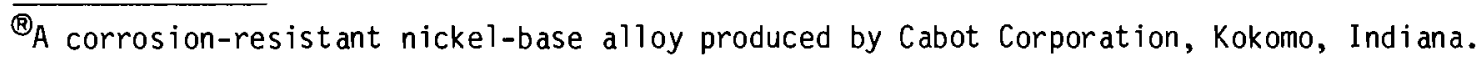




\subsection{MATERIALS EVALUATED}

\subsubsection{Material Selection}

A wide variety of inorganic materials can be considered for use in an engineered-barrier system. For a variety of reasons, the materials considered for evaluation in this study were limited to inorganic oxides and graphite. A literature search was conducted to determine what oxide materials might be expected to be most resistant to attack by the aqueous solutions that could be encountered in the two types of geologic waste repositories under consideration. In addition, recommendations on the types of materials to test were solicited from various individuals at PNL and Rockwell-Hanford who have the appropriate expertise. The materials finally selected for evaluation are listed in Table 1. The basalt evaluated in the study was taken from Flow $E$ of the Sentinel Bluffs-Grande Ronde Formations in south-central Washington. One grade of graphite was included in the screening tests. The graphite studied was Toyotanso ${ }^{\circledR}$ IG-11, which was developed for use in high-temperature, gas-cooled reactors and is reported to have a low permeability. Some naturally occurring, complex oxide minerals were seriously

TABLE 1. Materfals Evaluated in the Screening Tests

Material

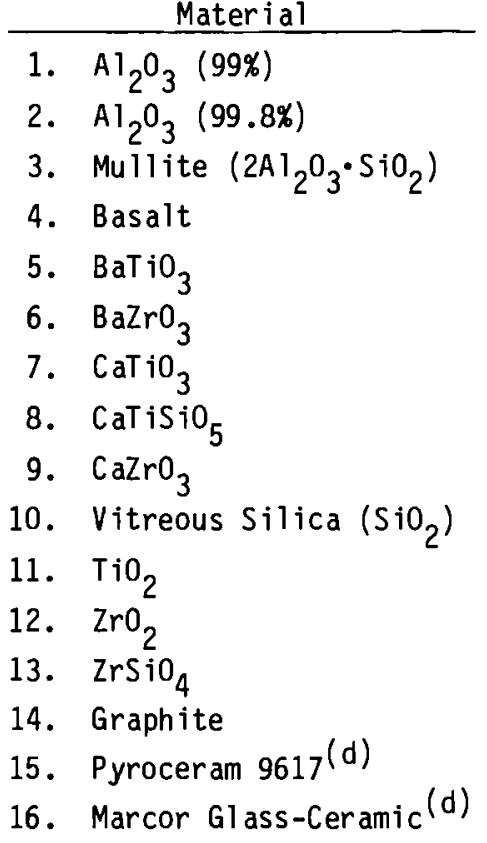

\begin{tabular}{l} 
Method Used to Fabricate \\
Dense Test Specimens \\
\hline Commercial \\
Commercial \\
Commercial \\
Naturally Occurring \\
Cold-Press and Sinter $(b)$ \\
Cold-Press and Sinter \\
Hot-Press \\
Cold-Press and Sinter \\
Cold-Press and Sinter \\
Commercial \\
Hot-Press \\
Commercial \\
Hot-Press \\
Commercial \\
Commercial \\
Commercial
\end{tabular}

(a) Obtained from a commercial source as a high-density shape.

(b) Powder cold-pressed into pellets and sintered.

(c) Powder hot-pressed into pellets.

(d) Corning Glass Works.

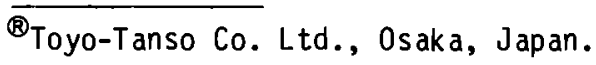


considered for evaluation but were dropped because they are being studied in other PNL programs. These include materials such as zirconolite, hollandite, and certain types of feldspars.

\subsubsection{Sample Preparation}

Wherever possible, the materials to be screened were obtained from commercial sources in the form of dense, impervious shapes. Several of the materials could only be obtained as powders and had to be compacted into dense test specimens. Depending on the material, hot-pressing or cold-pressing and sintering was used to fabricate the powders into dense pieces. Descriptions of the materials tested, including purity and method of densification, are given in the Appendix.

Fabricated shapes obtained from commercial sources and the naturally occurring basalt were sectioned into test specimens using a diamond saw. No attempt was made to polish the cut surfaces of the specimens before testing.

Hot-pressing was used to compact several of the powdered materials into high-density pellets. The hot press utilized an induction-heated graphite die and hydraulic press. The maximum temperature capability of the press was approximately $1700^{\circ} \mathrm{C}$, while the graphite die limited the maximum pressure to about $5800 \mathrm{psi}$. Pellets up to $1 \mathrm{in}$. in dia could be prepared by varying the die diameter. The maximum pellet length was about 1 in. Very little interaction was observed between the oxide pellets and the graphite die. The surface of the pellet, after removal from the die, was discolored with graphite that was easily removed by heating the pellet in air at about $1200^{\circ} \mathrm{C}$ for several hours.

The average densities of the hot-pressed pellets varied from about $86 \%$ of theoretical density for $\mathrm{ZrSiO}_{4}$ to $94 \%$ for $\mathrm{CaTiO}_{3}$. Density variations for pellets of a given material were about $\pm 0.5 \%$.

Attempts to hot-press $\mathrm{BaTiO}_{3}, \mathrm{BaZrO}_{3}, \mathrm{CaZrO}_{3}$, and $\mathrm{CaTiSiO}_{5}$ in graphite dies were unsuccessful. High-density pellets of the four materials were prepared by cold-pressing and sintering. One-inch-diameter pellets of each material were prepared by cold-pressing the powder at 50 tons $/$ in. $^{2}$ in a hardened split-sleeve steel die. The "green" pellets were then fired at approximately $1200^{\circ} \mathrm{C}$ for $90 \mathrm{~h}$ in an air-atmosphere muffle furnace. Average densities of the cold-pressed and sintered pellets ranged from $89 \%$ of theoretical density for $\mathrm{BaTi0}_{3}$ to $92 \%$ for $\mathrm{BaZrO}_{3}$. Density variations for pellets of a given material were about $\pm 1 \%$.

The pressed pellets were sectioned to the desired size using a diamond saw. The test specimens were cleaned, rinsed with demineralized water, and vacuum-dried at $180^{\circ} \mathrm{C}$. The specimens were then weighed on a semi-microbalance before testing.

Some of the test specimens (such as graphite) had considerable internal porosity and some were permeable. The surface areas of the specimens were determined by the Brunauer Emmett Teller (BET) method using krypton. 


\subsection{LEACH SOLUTIONS}

The candidate ceramic materials were leached in three different solutions: demineralized water, a synthetic Hanford ground water, and a synthetic brine solution. The compositions of the last two solutions are given in Table 2.

The demineralized water used in the leach tests was prepared using a Corning Model LD-5 water purifier. The treated water had a resistivity greater than $2 \mathrm{M} \Omega$, and its $\mathrm{pH}$ was approximately 6.2. Analysis of the demineralized water by induction-coupled plasma spectroscopy (ICP) showed that cation concentrations in the water were below the detectable limits for the instrument except for silicon, which averaged about $0.03 \mathrm{ppm}$.

The synthetic ground water used was based on the analysis of a ground water sample taken from the Umtanum basalt flow on the Hanford Reservation (Ames 1978). Analysis of groundwater samples taken from various sites on the reservation show significantly different compositions, but the composition used represents the one that has been widely used in various experimental programs at PNL, including radionuclide adsorption studies.

TABLE 2. Composition of Solutions Used in the Leaching Experiments

\begin{tabular}{|c|c|c|}
\hline 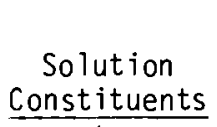 & $\begin{array}{l}\text { Synthet ic Hanford } \\
\text { Ground Water } \\
(\mathrm{pH}=8.0), \mathrm{mg} / \mathrm{L} \\
\end{array}$ & $\begin{array}{c}\text { Synthet ic Brine } \\
\text { Solution } \\
(\mathrm{pH}=6.5), \mathrm{mg} / \mathrm{L}(\mathrm{a}) \\
\end{array}$ \\
\hline $\mathrm{Na}^{+}$ & 33 & 115,000 \\
\hline$k^{+}$ & 9 & 18 \\
\hline $\mathrm{Ca}^{+2}$ & 6.5 & 900 \\
\hline $\mathrm{Mg}^{+2}$ & 1 & 10 \\
\hline $\mathrm{Sr}^{+2}$ & & 15 \\
\hline $\mathrm{Rb}^{+}$ & & 1 \\
\hline $\mathrm{Cs}^{+}$ & & 1 \\
\hline $\mathrm{Fe}^{+3}$ & & 2 \\
\hline $\mathrm{Cl}^{-}$ & 16 & 175,000 \\
\hline $\mathrm{SO}_{4}^{-2}$ & 23 & 4,200 \\
\hline $\mathrm{I}^{-}$ & & 10 \\
\hline $\mathrm{HCO}_{3}^{-}$ & 63 & 12 \\
\hline $\mathrm{F}^{-}$ & 0.7 & \\
\hline $\begin{array}{l}\mathrm{Br}^{-} \\
\mathrm{BO}_{3}^{-3}\end{array}$ & & $\begin{array}{r}400 \\
10\end{array}$ \\
\hline
\end{tabular}

(a) Synthetic solution density $022^{\circ} \mathrm{C}=1.18 \mathrm{~g} / \mathrm{mL}$. 
The synthetic salt brine used was a predominantly $\mathrm{NaCl}$ brine whose composition was representative of the brine encountered in the bedded salt at the WIPP site (Braithwaite and Molecke 1978). Brine of this composition has been and is being used in a variety of leaching tests at PNL and Sandia Laboratories.

\subsection{ANALYTICAL PROCEDURES}

Average leach rates were determined using the weight change measurements and solution analysis. The weight changes were monitored by weighing the test specimens on a semi-microbalance before and after testing. The various aqueous samples were analyzed for dissolved cations using an induction-coupled plasma spectrometer (ICP). Table 3 lists the detectable limits for various cations using the ICP. Analysis of the brine samples was complicated by the ir high $\mathrm{NaCl}$ content, but analysis of the samples at various dilutions gave fairly reproducible results.

After testing, the ceramic test specimens were subjected to ceramographic examination using standard techniques. The photomicrographs were used to determine if localized attack or alteration reactions had occurred during leaching.

TABLE 3. Detection Limits for Cations Using the Induction-Coupled Plasma Spectrometer

\begin{tabular}{|c|c|c|c|}
\hline Element & $\begin{array}{l}\text { Detection } \\
\text { Limit, ppm }\end{array}$ & Element & $\begin{array}{l}\text { Detection } \\
\text { Limit, ppm }\end{array}$ \\
\hline$A 1$ & 0.013 & Mg & 0.005 \\
\hline As & 0.032 & $\mathrm{Mn}$ & 0.001 \\
\hline$B$ & 0.004 & Mo & 0.003 \\
\hline $\mathrm{Ba}$ & 0.001 & $\mathrm{Na}$ & 0.17 \\
\hline $\mathrm{Ca}$ & 0.005 & Nd & 0.005 \\
\hline $\mathrm{Cd}$ & 0.002 & $\mathrm{Ni}$ & 0.007 \\
\hline $\mathrm{Ce}$ & 0.009 & $P$ & 0.047 \\
\hline Co & 0.002 & $\mathrm{~Pb}$ & 0.009 \\
\hline $\mathrm{Cr}$ & 0.006 & $\mathrm{Ru}$ & 0.007 \\
\hline $\mathrm{Cu}$ & 0.001 & Sb & 0.016 \\
\hline Dy & 0.001 & Si & 0.005 \\
\hline Eu & 0.001 & Sr & 0.001 \\
\hline $\mathrm{Fe}$ & 0.003 & $\mathrm{Te}$ & 0.02 \\
\hline Gd & 0.004 & $\mathrm{Ti}$ & 0.001 \\
\hline K & 0.041 & $U$ & 0.04 \\
\hline La & 0.020 & $\mathrm{Zn}$ & 0.004 \\
\hline $\mathrm{Li}$ & 0.002 & $\mathrm{Zr}$ & 0.001 \\
\hline
\end{tabular}




\subsection{RESULTS AND DISCUSSION}

A primary objective of this study was to screen a large number of candidate ceramic materials to determine which materials would be most resistant to attack in the repository environments. The principal method of material screening involved subjecting test specimens of each material to a variety of leach tests and determining their relative resistances to attack. An initial comparison of the materials can be obtained by determining their average leach rates under the various test conditions. The average leach rates can be determined from weight change measurements and from analyses of the leach solutions. Average leach rates can be misleading, however; localized attack of the test specimens, such as the leaching of impurities from grain boundaries, can result in far more serious attack than is evident from average leach rate determinations. In addition, alteration reactions at the specimen's surface can result in extensive attack that may not be apparent from either the solution analyses or the weight change measurements.

Determining if localized attack or alteration reactions are a problem requires that the ceramic specimens be subjected, after testing, to additional analyses such as ceramographic examination and scanning electron microscopy (SEM). Because of the large number of specimens tested it was necessary to limit the analyses to ceramographic examination of selected specimens--primarily those tested at the higher temperatures.

The photomicrographs obtained showed that several of the ceramic materials were susceptible to localized attack or alteration reactions. For this reason, the average leach rate data presented in Section 4.1 should not be used as a final bas is for ranking the candidate materials. The final comparison and ranking of the materials must consider the effect of localized attack and alteration reactions, as well as a number of other factors including:

- cost

- availability

- mechanical and physical properties, and

- the availability of demonstrated techniques for fabricating dense, impervious shapes of the size required for an engineered-barrier system.

A detafled literature search on the properties of the ceramic materials under consideration was not carried out, and the comparison of the candidates was limited to the data given in various handbooks, such as Engineering Properties of Selected Ceramic Materials (Lynch, Ruderer and Duckworth 1966). Each of the ceramic materials is discussed individually in Section 4.3.

\subsection{LEACH RATES}

The average leach rates for the ceramic materials tested were determined from the solution analysis data and/or weight loss measurements. Calculation of the leach rates was based 
on the geometric surface areas of the test specimens. With some of the materials there were differences in the leach rates determined from the two types of data. In general, the discrepancies were relatively greater for the more leach-resistant materials such as aluminum oxide than for the more soluble materials such as Pyroceram 9617.' In most cases the leach rates determined from weight loss measurements were greater than those calculated from the solution analyses. Unless otherwise noted, the leach rates given in the following tables represent the maximum values determined from the two methods.

Two other factors affect the average leach rate data given in the tables. All of the test specimens had some porosity. Some were impervious; others, however, were somewhat permeable and the effective areas available for leaching were much greater than were the respective geometric surface areas of the test specimens used to calculate the average leach rates. For example, the effective surface area of a typical graphite test specimen, as determined by the BET method, was about $7000 \mathrm{~cm}^{2}$, while the geometric surface area was about $3 \mathrm{~cm}^{2}$.

The permeability of the specimens presented an additional problem when they were leached in the brine solution. At the conclusion of a test, it was very difficult to completely wash the brine solution from the interstices of the permeable specimens--especially the graphite. When the specimens were vacuum-dried before weighing, the dissolved solids in the residual brine precipitated in the voids, affecting the final weight measurement. In some cases, it was necess ary to wash the specimens several times before a constant weight was attained, and even then there was no assurance that all of the dissolved solids in the brine had been completely removed from the specimens. Since the leach rates were usually very low, the presence of a small amount of residue from the brine would have an effect on the weight change measurements.

\subsubsection{Static Leach Tests at $100^{\circ} \mathrm{C}$}

The average leach rates for ceramic samples exposed to demineralized water at $100^{\circ} \mathrm{C}$ in a static vented system are given in Table 4. The leach rates were determined from weight loss measurements. Analysis of the leach solution proved to be an unreliable method for determining the leach rates in most instances because of the leaching of material from the glassware used in the tests.

The tests were carried out in Pyrex glassware. The exposed surface of the glassware was many $t$ imes greater than the geometric surface area of the ceramic test specimen, and leaching of the glassware could have had a marked effect on the leaching of the ceramic sample. In a typical leach test the $S / V$ ratio for the ceramic sample was about $0.02 \mathrm{~cm}^{-1}$, whereas the $S / V$ ratio for the glass ware was about $1.0 \mathrm{~cm}^{-1}$. The principal cation leached from the Pyrex glassware was silicon, together with lesser amounts of boron, sodium, aluminum, potassium, and calcium. Table 5 shows the range of results obtained when blank leach tests were run with the glassware alone. The cations leached from the glassware were also present in most of the ceramic materials tested either as primary constituents or impurities. This could affect the 
TABLE 4. Average Leach Rates for Ceramic Materials in Demineralized Water at $100^{\circ} \mathrm{C}--$ Vented Static System

\begin{tabular}{|c|c|c|c|}
\hline Material & $\begin{array}{c}\text { Exposure, } \\
\text { days }\end{array}$ & $\begin{array}{l}\text { Average Leach } \\
\text { Overall }(\mathrm{c})\end{array}$ & 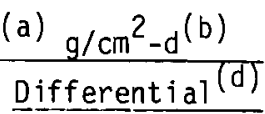 \\
\hline Graphite & $\begin{array}{l}14 \\
24 \\
36 \\
43\end{array}$ & $\begin{array}{l}6.8 \times 10^{-7} \\
5.3 \times 10^{-7} \\
5.3 \times 10^{-7} \\
5.9 \times 10^{-7}\end{array}$ & $\begin{array}{l}6.8 \times 10^{-7} \\
3.2 \times 10^{-7} \\
6.3 \times 10^{-7} \\
9.1 \times 10^{-7}\end{array}$ \\
\hline $\mathrm{Al}_{2} \mathrm{O}_{3} \quad(99.8 \%)$ & $\begin{array}{l}14 \\
24 \\
36 \\
43\end{array}$ & $\begin{array}{l}2.0 \times 10^{-6} \\
1.6 \times 10^{-6} \\
1.5 \times 10^{-6} \\
1.4 \times 10^{-6}\end{array}$ & $\begin{array}{l}2.0 \times 10^{-6} \\
0.9 \times 10^{-6} \\
1.6 \times 10^{-6} \\
1.1 \times 10^{-6}\end{array}$ \\
\hline $\mathrm{Al}_{2} \mathrm{O}_{3} \quad(99 \%)$ & $\begin{array}{l}14 \\
24 \\
36 \\
43\end{array}$ & $\begin{array}{l}7.5 \times 10^{-6} \\
7.0 \times 10^{-6} \\
5.7 \times 10^{-6} \\
5.3 \times 10^{-6}\end{array}$ & $\begin{array}{l}7.5 \times 10^{-6} \\
6.4 \times 10^{-6} \\
3.0 \times 10^{-6} \\
3.2 \times 10^{-6}\end{array}$ \\
\hline Pyroceram 9617 & $\begin{array}{l}14 \\
24 \\
36 \\
43\end{array}$ & $\begin{array}{l}1.7 \times 10^{-5} \\
1.4 \times 10^{-5} \\
1.4 \times 10^{-5} \\
1.4 \times 10^{-5}\end{array}$ & $\begin{array}{l}1.7 \times 10^{-5} \\
1.1 \times 10^{-5} \\
1.4 \times 10^{-5} \\
1.3 \times 10^{-5}\end{array}$ \\
\hline $\begin{array}{l}\text { Vitreous Silica } \\
\left(\mathrm{SiO}_{2}\right)\end{array}$ & $\begin{array}{l}14 \\
28 \\
35 \\
49\end{array}$ & $\begin{array}{l}2.6 \times 10^{-5} \\
1.2 \times 10^{-5} \\
1.0 \times 10^{-5} \\
8.2 \times 10^{-6}\end{array}$ & $\begin{array}{l}2.6 \times 10^{-5} \\
1.0 \times 10^{-6} \\
1.4 \times 10^{-6} \\
1.3 \times 10^{-6}\end{array}$ \\
\hline $\mathrm{TiO}_{2}$ & $\begin{array}{l}14 \\
28 \\
35 \\
49\end{array}$ & $\begin{array}{l}5.2 \times 10^{-5} \\
2.6 \times 10^{-5} \\
2.1 \times 10^{-5} \\
1.5 \times 10^{-5}\end{array}$ & $\begin{array}{l}5.2 \times 10^{-5} \\
1.1 \times 10^{-6} \\
4.5 \times 10^{-7} \\
7.8 \times 10^{-7}\end{array}$ \\
\hline Basalt & $\begin{array}{l}14 \\
28 \\
35 \\
49\end{array}$ & $\begin{array}{l}5.8 \times 10^{-5} \\
4.9 \times 10^{-5} \\
3.5 \times 10^{-5} \\
3.9 \times 10^{-5}\end{array}$ & $\begin{array}{l}5.8 \times 10^{-5} \\
4.0 \times 10^{-5} \\
3.9 \times 10^{-6} \\
3.7 \times 10^{-5}\end{array}$ \\
\hline $\mathrm{CaTi0} 3$ & $\begin{array}{l}14 \\
28 \\
35 \\
49\end{array}$ & $\begin{array}{l}5.4 \times 10^{-5} \\
2.8 \times 10^{-5}(\mathrm{e}) \\
2.3 \times 10^{-5} \\
1.8 \times 10^{-5}\end{array}$ & $\begin{array}{l}5.4 \times 10^{-5} \\
2.2 \times 10^{-6} \\
3.9 \times 10^{-6} \\
3.2 \times 10^{-6}\end{array}$ \\
\hline $\mathrm{ZrO}_{2}$ & $\begin{array}{l}14 \\
28 \\
35 \\
49\end{array}$ & $\begin{array}{l}6.2 \times 10^{-5} \\
5.5 \times 10^{-5} \\
4.8 \times 10^{-5} \\
4.2 \times 10^{-5}\end{array}$ & $\begin{array}{l}6.2 \times 10^{-5} \\
4.8 \times 10^{-5} \\
3.1 \times 10^{-5} \\
1.5 \times 10^{-5}\end{array}$ \\
\hline $\mathrm{ZrSiO}_{4}$ & $\begin{array}{l}14 \\
28 \\
35 \\
49\end{array}$ & $\begin{array}{l}7.3 \times 10^{-5} \\
4.9 \times 10^{-5} \\
4.5 \times 10^{-5} \\
4.3 \times 10^{-5}\end{array}$ & $\begin{array}{l}7.3 \times 10^{-5} \\
2.4 \times 10^{-5} \\
3.1 \times 10^{-5} \\
3.3 \times 10^{-5}\end{array}$ \\
\hline Marcor & $\begin{array}{l}14 \\
28 \\
35 \\
49\end{array}$ & $\begin{array}{l}7.5 \times 10^{-5} \\
5.8 \times 10^{-5} \\
5.4 \times 10^{-5} \\
4.8 \times 10^{-5}\end{array}$ & $\begin{array}{l}7.5 \times 10^{-5} \\
4.1 \times 10^{-5} \\
3.8 \times 10^{-5} \\
3.2 \times 10^{-5}\end{array}$ \\
\hline
\end{tabular}


TABLE 4. (contd)

\begin{tabular}{|c|c|c|c|}
\hline Material & $\begin{array}{c}\text { Exposure, } \\
\text { days }\end{array}$ & \multicolumn{2}{|c|}{ Average Leach Rate, ${ }^{(a)} \mathrm{g} / \mathrm{cm}^{2}-d^{(b)}$} \\
\hline $\mathrm{BaTiO}_{3}$ & $\begin{array}{l}14 \\
28 \\
35 \\
49\end{array}$ & $\begin{array}{r}13.0 \times 10^{-5} \\
7.6 \times 10^{-5} \\
6.3 \times 10^{-5} \\
4.8 \times 10^{-5}\end{array}$ & $\begin{array}{r}13.0 \times 10^{-5} \\
2.0 \times 10^{-5} \\
1.3 \times 10^{-5} \\
1.1 \times 10^{-5}\end{array}$ \\
\hline $\mathrm{BaZrO}_{3}$ & $\begin{array}{l}14 \\
28 \\
35 \\
49\end{array}$ & $\begin{aligned} 13.0 \times 10^{-5} \\
8.5 \times 10^{-5} \\
7.0 \times 10^{-5}(\mathrm{e}) \\
5.5 \times 10^{-5}\end{aligned}$ & $\begin{array}{r}13.0 \times 10^{-5} \\
3.5 \times 10^{-5} \\
1.4 \times 10^{-5} \\
3.3 \times 10^{-5}\end{array}$ \\
\hline $\mathrm{CaTiSiO} 5$ & $\begin{array}{l}14 \\
28 \\
35 \\
49\end{array}$ & $\begin{array}{l}2.8 \times 10^{-4} \\
1.9 \times 10^{-4} \\
1.6 \times 10^{-4}(\mathrm{e}) \\
1.6 \times 10^{-4}\end{array}$ & $\begin{array}{l}2.8 \times 10^{-4} \\
8.9 \times 10^{-5} \\
6.1 \times 10^{-5} \\
1.4 \times 10^{-4}\end{array}$ \\
\hline $\begin{array}{l}\text { Mullite } \\
\left(2 \mathrm{Al}_{2} \mathrm{O}_{3} \cdot \mathrm{SiO}_{2}\right)\end{array}$ & $\begin{array}{l}14 \\
24 \\
36 \\
43\end{array}$ & $\begin{array}{l}3.1 \times 10^{-4} \\
3.2 \times 10^{-4} \\
3.0 \times 10^{-4} \\
2.6 \times 10^{-4}\end{array}$ & $\begin{array}{l}3.1 \times 10^{-4} \\
3.2 \times 10^{-4} \\
2.8 \times 10^{-4} \\
1.2 \times 10^{-6}\end{array}$ \\
\hline $\mathrm{CaZrO}_{3}$ & $\begin{array}{l}14 \\
28 \\
35 \\
49\end{array}$ & $\begin{array}{l}3.2 \times 10^{-5} \\
1.5 \times 10^{-4}(\mathrm{e}) \\
1.7 \times 10^{-4} \\
2.5 \times 10^{-4}\end{array}$ & $\begin{array}{l}3.2 \times 10^{-5} \\
3.0 \times 10^{-4} \\
1.9 \times 10^{-4} \\
4.5 \times 10^{-4}\end{array}$ \\
\hline
\end{tabular}

(a) Determined from weight loss measurements.

(b) Based on the geometric surface area of the test specimen.

(c) Average leach rate for the exposure time shown.

(d) Average leach rate for specific time interval.

(e) Solution turned cloudy.

leaching of some materials--especially the vitreous silica, mullite, $\mathrm{ZrSiO}_{4}$ and $\mathrm{CaTiSiO}_{5}$. Leaching of components from the glassware would also affect the pH of the leach solution, which could affect the leaching of the different materials.

The leach rates for the materials tested varied by about a factor of 500 , with graphite exhibiting a significantly lower leach rate than any of the other materials. In several of the tests the leach solutions turned cloudy, indicating that some disintegration of the test specimen had occurred. When the specimens from these tests were vacuum-dried, they exhibited a powdery surface texture. All of the tests in which the solutions turned cloudy involved specimens that had been prepared at PNL by hot-pressing or cold-pressing and sintering. Time did not permit a detailed study to determine if the leach resistance of the materials could be increased by using different or improved procedures for fabricating the test specimens.

One surprising factor was the relatively high leach rate exhibited by the mullite. Overall, it exhibited the highest leach rate of any of the materials tested. In all of the other leach tests, however, mullite was average or better than average in leach resistance. No reason for the high leach rate of the mullite at $100^{\circ} \mathrm{C}$ in demineralized water was identified. 
TABLE 5. Leaching of Pyrex Glassware in Demineralized Water at $100^{\circ} \mathrm{C}$

1. Static Vented System

2. Leaching Time - $43 \mathrm{~d}(1032 \mathrm{~h})$

3. Exposed $G 1$ ass Surface Area/Solution Volume $\left(S / V \cong 1.0 \mathrm{~cm}^{-1}\right)$

4. Final Analys is of Leach Solutions (range of values for triplicate experiments):

$$
\begin{array}{rlrl}
\mathrm{Si} & =20 \text { to } 50 \mathrm{ppm} & \mathrm{Al} & =1 \text { to } 2 \mathrm{ppm} \\
\mathrm{B} & =2 \text { to } 5 \mathrm{ppm} & \mathrm{K} & =0.2 \text { to } 1 \mathrm{ppm} \\
\mathrm{Na} & =1 \text { to } 3 \mathrm{ppm} & \mathrm{Ca} & =0.1 \text { to } 0.5 \mathrm{ppm} .
\end{array}
$$

In most of the tests the leach rates were initially high but decreased with time. This is to be expected with static leach tests involving materials whose components have a low solubility in water.

\subsubsection{Dynamic Leach Tests at $90^{\circ} \mathrm{C}$}

The average leach rates for ceramic materials exposed to flowing demineralized water at $90^{\circ} \mathrm{C}$ are given in Table 6 . The leach rates were determined from the weight loss measurements. The concentrations of dissolved species in the leach solutions leaving the reaction vessels were too low to permit reliable determination of the leach rates.

The leach rates for the various materials varied by a factor of about 250 , with graphite exhibiting the lowest leach rate. Overall, the materials exhibited slightly lower leach rates in the dynamic tests at $90^{\circ} \mathrm{C}$ than in static tests at $100^{\circ} \mathrm{C}$. The mullite, however, exhibited a leach rate about one hundred times lower than the rate at $100^{\circ} \mathrm{C}$. Most of the materials exhibited a high initial leach rate that decreased with time, and some appeared to achieve a relatively constant rate after prolonged exposure. One material, the Marcor machinable glassceramic, exhibited a leach rate that appeared to be increasing with time.

\subsubsection{Dynamic Leach Tests at $250^{\circ} \mathrm{C}$}

Samples of the ceramic materials were exposed to flowing demineralized water at $250^{\circ} \mathrm{C}$ in an autoclave. The sixteen materials were tested in two groups of eight. Each group of specimens was tested for four time periods. The testing sequence for the first group was 7 days, 14 days, 21 days, and 1 day; for the second group it was 23 days, 7 days, 14 days, and 1 day. After the final test each specimen was subjected to ceramographic examination. Limited autoclave availability and other scheduling problems made it necessary to change the testing sequence for the second group of materials. The differences in the testing sequences for the two groups of materials should have little effect on relative leach rates of the materials. Possible interactions between each group tested should have little effect on the results obtained.

The average leach rates were calculated from the weight loss measurements, and the results obtained are given in Table 7. The concentrations of dissolved species in the leach solution leaving the autoclave were too low to permit reliable determination of leach rates from solution analyses. In addition, eight materials--all of which contained common cations either 
TABLE 6. Leaching of Ceramic Materials in Flowing Demineralized Water at $90^{\circ} \mathrm{C}$

\begin{tabular}{|c|c|c|c|c|}
\hline Material & $\begin{array}{l}\text { Time, } \\
\text { days }\end{array}$ & & $\frac{\text { Average Leac }}{\text { Overall }(\mathrm{c})}$ & Average Leach Rate, $(a) g / \mathrm{cm}^{2}-d^{(b)}$ \\
\hline Graphite 2.2 & $\begin{array}{r}7 \\
14 \\
21 \\
28 \\
35\end{array}$ & $\because 6$ & $\begin{array}{r}5.8 \times 10^{-7} \\
3.8 \times 10^{-7} \\
4.1 \times 10^{-7} \\
4.0 \times 10^{-7} \\
4.0 \times 10^{-7}\end{array}$ & $\begin{array}{l}5.8 \times 10^{-7} \\
2.3 \times 10^{-7} \\
2.7 \times 10^{-7} \\
2.6 \times 10^{-7} \\
2.7 \times 10^{-7}\end{array}$ \\
\hline $\mathrm{Al}_{2} \mathrm{O}_{3}(99.8 \%)$ & $\begin{array}{r}7 \\
14 \\
21 \\
28 \\
35\end{array}$ & 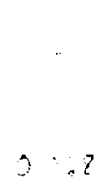 & $\begin{array}{r}16.0 \times 10^{-7} \\
11.0 \times 10^{-7} \\
8.9 \times 10^{-7} \\
6.9 \times 10^{-7} \\
7.4 \times 10^{-7}\end{array}$ & $\begin{array}{r}10.0 \times 10^{-7} \\
11.0 \times 10^{-7} \\
5.5 \times 10^{-7} \\
5.7 \times 10^{-7} \\
10.0 \times 10^{-7}\end{array}$ \\
\hline $\begin{array}{l}\text { Mullite } \\
\left(2 \mathrm{Al}_{2} \mathrm{O}_{3} \cdot \mathrm{SiO}_{2}\right)\end{array}$ & $\begin{array}{l}7 \\
14 \\
21 \\
28 \\
35\end{array}$ & & $\begin{array}{l}4.3 \times 10^{-6} \\
2.9 \times 10^{-6} \\
2.3 \times 10^{-6} \\
2.1 \times 10^{-6} \\
2.4 \times 10^{-6}\end{array}$ & $\begin{array}{l}4.3 \times 10^{-6} \\
1.4 \times 10^{-6} \\
1.4 \times 10^{-6} \\
1.4 \times 10^{-6} \\
3.5 \times 10^{-6}\end{array}$ \\
\hline $\mathrm{Al}_{2} \mathrm{O}_{3}(99 \%)$ & $\begin{array}{r}7 \\
14 \\
21 \\
28 \\
35\end{array}$ & & $\begin{array}{l}5.4 \times 10^{-6} \\
4.0 \times 10^{-6} \\
3.6 \times 10^{-6} \\
3.4 \times 10^{-6} \\
3.5 \times 10^{-6}\end{array}$ & $\begin{array}{l}3.4 \times 10^{-6} \\
2.7 \times 10^{-6} \\
2.7 \times 10^{-6} \\
2.7 \times 10^{-6} \\
4.0 \times 10^{-6}\end{array}$ \\
\hline $\mathrm{TiO}_{2} \perp 2=$ & $\begin{array}{r}7 \\
14 \\
21 \\
28 \\
35\end{array}$ & 0.33 & $\begin{array}{r}11.0 \times 10^{-6} \\
6.7 \times 10^{-6} \\
5.0 \times 10^{-6} \\
4.3 \times 10^{-6} \\
3.8 \times 10^{-6}\end{array}$ & $\begin{array}{r}11.0 \times 10^{-6} \\
2.8 \times 10^{-6} \\
1.9 \times 10^{-6}-6 \\
2.1 \times 10^{-6} \\
1.8 \times 10^{-6}\end{array}$ \\
\hline $\mathrm{ZrO}_{2}$ & $\begin{array}{r}7 \\
14 \\
21 \\
28 \\
35\end{array}$ & $67^{2}$ & $\begin{array}{l}3.6 \times 10^{-5} \\
2.3 \times 10^{-5} \\
1.7 \times 10^{-5} \\
1.4 \times 10^{-5} \\
1.1 \times 10^{-5}\end{array}$ & $\begin{array}{r}3.6 \times 10^{-5} \\
0.96 \times 10^{-5} \\
0.48 \times 10^{-5} \\
0.43 \times 10^{-5} \\
0.25 \times 10^{-5}\end{array}$ \\
\hline Pyroceram 9617 & $\begin{array}{l}7 \\
14 \\
21 \\
28 \\
35\end{array}$ & & $\begin{array}{r}0.47 \times 10^{-5} \\
1.6 \times 10^{-5} \\
1.4 \times 10^{-5} \\
1.3 \times 10^{-5} \\
1.2 \times 10^{-5}\end{array}$ & $\begin{array}{r}0.47 \times 10^{-5} \\
2.7 \times 10^{-5} \\
0.95 \times 10^{-5} \\
1.2 \times 10^{-5} \\
0.62 \times 10^{-5}\end{array}$ \\
\hline $\mathrm{CaTiO}_{3}$ & $\begin{array}{l}7 \\
14 \\
21 \\
28 \\
35\end{array}$ & & $\begin{array}{l}6.4 \times 10^{-5} \\
5.3 \times 10^{-5} \\
4.5 \times 10^{-5} \\
4.0 \times 10^{-5} \\
3.5 \times 10^{-5}\end{array}$ & $\begin{array}{l}6.4 \times 10^{-5} \\
4.1 \times 10^{-5} \\
2.9 \times 10^{-5} \\
2.5 \times 10^{-5} \\
1.5 \times 10^{-5}\end{array}$ \\
\hline $\mathrm{CaZrO}_{3}$ & $\begin{array}{r}7 \\
14 \\
21 \\
28 \\
35\end{array}$ & & $\begin{array}{l}6.5 \times 10^{-5} \\
5.7 \times 10^{-5} \\
4.8 \times 10^{-5} \\
4.5 \times 10^{-5} \\
4.7 \times 10^{-5}\end{array}$ & $\begin{array}{l}6.4 \times 10^{-5} \\
4.9 \times 10^{-5} \\
2.9 \times 10^{-5} \\
3.5 \times 10^{-5} \\
5.6 \times 10^{-5}\end{array}$ \\
\hline
\end{tabular}


TABLE 6. (contd)

\begin{tabular}{|c|c|c|c|}
\hline \multirow[b]{2}{*}{ Material } & \multirow{2}{*}{$\begin{array}{l}\text { Time, } \\
\text { days }\end{array}$} & \multicolumn{2}{|c|}{ Average Leach Rate, (a) $\mathrm{g} / \mathrm{cm}^{2}-d^{\text {(b) }}$} \\
\hline & & Overall $(c)$ & Differential ${ }^{(d)}$ \\
\hline Basalt & $\begin{array}{r}7 \\
14 \\
21 \\
28 \\
35\end{array}$ & $\begin{array}{l}7.7 \times 10^{-5} \\
6.7 \times 10^{-5} \\
4.6 \times 10^{-5} \\
3.6 \times 10^{-5} \\
3.4 \times 10^{-5}\end{array}$ & $\begin{array}{r}7.7 \times 10^{-5} \\
5.8 \times 10^{-5} \\
0.35 \times 10^{-5} \\
0.69 \times 10^{-5} \\
2.4 \times 10^{-5}\end{array}$ \\
\hline $\begin{array}{l}\text { Vitreous Silica } \\
\left(\mathrm{SiO}_{2}\right)\end{array}$ & $\begin{array}{r}7 \\
14 \\
21 \\
28 \\
35\end{array}$ & $\begin{array}{l}7.1 \times 10^{-5} \\
5.3 \times 10^{-5} \\
5.5 \times 10^{-5} \\
5.1 \times 10^{-5} \\
4.9 \times 10^{-5}\end{array}$ & $\begin{array}{l}7.1 \times 10^{-5} \\
3.5 \times 10^{-5} \\
5.9 \times 10^{-5} \\
4.1 \times 10^{-5} \\
3.8 \times 10^{-5}\end{array}$ \\
\hline Marcor & $\begin{array}{r}7 \\
14 \\
21 \\
28 \\
35\end{array}$ & $\begin{array}{l}5.4 \times 10^{-5} \\
5.1 \times 10^{-5} \\
4.9 \times 10^{-5} \\
5.5 \times 10^{-5} \\
6.3 \times 10^{-5}\end{array}$ & $\begin{array}{l}5.4 \times 10^{-5} \\
4.7 \times 10^{-5} \\
4.4 \times 10^{-5} \\
7.6 \times 10^{-5} \\
9.2 \times 10^{-5}\end{array}$ \\
\hline $\mathrm{ZrSiO}_{4}$ & $\begin{array}{r}7 \\
14 \\
21 \\
28 \\
35\end{array}$ & $\begin{array}{l}8.9 \times 10^{-5} \\
7.8 \times 10^{-5} \\
7.3 \times 10^{-5} \\
7.0 \times 10^{-5} \\
6.8 \times 10^{-5}\end{array}$ & $\begin{array}{l}8.9 \times 10^{-5} \\
6.8 \times 10^{-5} \\
6.3 \times 10^{-5} \\
6.0 \times 10^{-5} \\
5.8 \times 10^{-5}\end{array}$ \\
\hline $\mathrm{BaTiO} 3$ & $\begin{array}{r}7 \\
14 \\
21 \\
28 \\
35\end{array}$ & $\begin{array}{r}11.6 \times 10^{-5} \\
11.0 \times 10^{-5} \\
9.2 \times 10^{-5} \\
8.9 \times 10^{-5} \\
8.6 \times 10^{-5}\end{array}$ & $\begin{array}{r}11.6 \times 10^{-5} \\
10.3 \times 10^{-5} \\
6.4 \times 10^{-5} \\
7.8 \times 10^{-5} \\
7.3 \times 10^{-5}\end{array}$ \\
\hline CaTiSi0 5 & $\begin{array}{r}7 \\
14 \\
21 \\
28 \\
35\end{array}$ & $\begin{array}{r}11.1 \times 10^{-5} \\
8.7 \times 10^{-5} \\
8.6 \times 10^{-5} \\
8.1 \times 10^{-5} \\
7.8 \times 10^{-5}\end{array}$ & $\begin{array}{r}11.1 \times 10^{-5} \\
6.2 \times 10^{-5} \\
7.7 \times 10^{-5} \\
6.7 \times 10^{-5} \\
6.7 \times 10^{-5}\end{array}$ \\
\hline $\mathrm{BaZrO}_{3}$ & $\begin{array}{r}7 \\
14 \\
21 \\
28 \\
35\end{array}$ & $\begin{array}{r}13.0 \times 10^{-5} \\
11.0 \times 10^{-5} \\
10.0 \times 10^{-5} \\
9.9 \times 10^{-5} \\
9.6 \times 10^{-5}\end{array}$ & $\begin{array}{r}13.0 \times 10^{-5} \\
9.2 \times 10^{-5} \\
9.4 \times 10^{-5} \\
8.2 \times 10^{-5} \\
8.0 \times 10^{-5}\end{array}$ \\
\hline
\end{tabular}

(a) Determined from weight loss measurements.

(b) Based on the geometric surface area of the test specimen.

(c) Average leach rate for the exposure time shown.

(d) Differential leach rate for the specific time interval.

as primary constituents or impurities--were tested in the autoclave at one time, which precluded the use of solution analys is to determine leach rates.

Leach rates for the materials tested varied by about a factor of 1500 , with graphite exhibiting the lowest leach rate. Titanium dioxide also exhibited a very low rate. In fact, the leach rate for $\mathrm{TiO}_{2}$ at $250^{\circ} \mathrm{C}$ in flowing demineralized water was about the same as was its rate at $90^{\circ} \mathrm{C}$. The other materials tested at $250^{\circ} \mathrm{C}$ had much higher leach rates than did the 
TABLE 7. Average Leach Rates for Ceramic Materials Exposed to Flowing Demineralized Water at $250^{\circ} \mathrm{C}(\mathrm{a})$

\begin{tabular}{|c|c|c|c|c|c|}
\hline \multicolumn{2}{|l|}{ Material } & $\begin{array}{c}\text { Exposure, } \\
\text { days }\end{array}$ & \multicolumn{2}{|c|}{ Average Leach Rate, ${ }^{(b)} \mathrm{g} / \mathrm{cm}^{2}-d(\mathrm{c})$} & Remarks \\
\hline Graphite & & $\begin{array}{r}7 \\
21 \\
42 \\
43\end{array}$ & $\begin{array}{l}2.0 \times 10^{-6} \\
5.7 \times 10^{-6} \\
6.9 \times 10^{-6} \\
7.0 \times 10^{-6}\end{array}$ & $\begin{array}{l}2.0 \times 10^{-6} \\
7.6 \times 10^{-6} \\
8.1 \times 10^{-6} \\
9.0 \times 10^{-6}\end{array}$ & \\
\hline $\mathrm{TiO}_{2}$ & & $\begin{array}{l}23 \\
30 \\
44 \\
45\end{array}$ & $\begin{array}{l}9.6 \times 10^{-6} \\
7.5 \times 10^{-6} \\
6.3 \times 10^{-6} \\
6.7 \times 10^{-6}\end{array}$ & $\begin{array}{r}9.6 \times 10^{-6} \\
1.0 \times 10^{-6} \\
3.9 \times 10^{-6} \\
22.2 \times 10^{-6}\end{array}$ & Radial Cracks \\
\hline $\mathrm{ZrO}_{2}$ & 0.2 & $\begin{array}{r}7 \\
21 \\
42 \\
43\end{array}$ & $\begin{array}{l}3.0 \times 10^{-4} \\
1.6 \times 10^{-4} \\
1.0 \times 10^{-4} \\
1.0 \times 10^{-4}\end{array}$ & $\begin{array}{l}3.0 \times 10^{-4} \\
9.2 \times 10^{-5} \\
3.9 \times 10^{-5} \\
1.0 \times 10^{-5}\end{array}$ & \\
\hline $\mathrm{Al}_{2} \mathrm{O}_{3}(99.8 \%)$ & $0_{0} 1$ & $\begin{array}{r}7 \\
21 \\
42 \\
43\end{array}$ & $\begin{array}{l}1.4 \times 10^{-4} \\
1.2 \times 10^{-4} \\
1.3 \times 10^{-4} \\
1.3 \times 10^{-4}\end{array}$ & $\begin{array}{l}1.4 \times 10^{-4} \\
1.1 \times 10^{-4} \\
1.5 \times 10^{-4} \\
1.6 \times 10^{-4}\end{array}$ & Localized Attack \\
\hline $\mathrm{Al}_{2} \mathrm{O}_{3} \quad(99 \%)$ & .2 & $\begin{array}{r}7 \\
21 \\
42 \\
43\end{array}$ & $\begin{array}{l}2.5 \times 10^{-4} \\
1.9 \times 10^{-4} \\
1.7 \times 10^{-4} \\
1.8 \times 10^{-4}\end{array}$ & $\begin{array}{l}2.5 \times 10^{-4} \\
1.5 \times 10^{-4} \\
1.5 \times 10^{-4} \\
2.6 \times 10^{-4}\end{array}$ & Localized Attack \\
\hline $\begin{array}{l}\text { Vitreous Silica } \\
\left(\mathrm{SiO}_{2}\right)\end{array}$ & 25 & $\begin{array}{r}7 \\
21 \\
42 \\
43\end{array}$ & $\begin{array}{l}6.9 \times 10^{-4} \\
5.3 \times 10^{-4} \\
4.9 \times 10^{-4} \\
5.0 \times 10^{-4}\end{array}$ & $\begin{array}{l}6.9 \times 10^{-4} \\
4.5 \times 10^{-4} \\
4.4 \times 10^{-4} \\
5.1 \times 10^{-4}\end{array}$ & \\
\hline $\mathrm{CaZrO}_{3}$ & $\because$ & $\begin{array}{l}23 \\
30 \\
44 \\
45\end{array}$ & $\begin{array}{l}3.5 \times 10^{-4} \\
4.8 \times 10^{-4} \\
4.4 \times 10^{-4} \\
4.4 \times 10^{-4}\end{array}$ & $\begin{array}{l}3.5 \times 10^{-4} \\
9.0 \times 10^{-4} \\
3.5 \times 10^{-4} \\
4.8 \times 10^{-4}\end{array}$ & Alteration Reaction \\
\hline CaTiSi0 5 & 0.6 & $\begin{array}{l}23 \\
30 \\
44 \\
45\end{array}$ & $\begin{array}{l}4.5 \times 10^{-4} \\
5.3 \times 10^{-4} \\
6.1 \times 10^{-4} \\
7.0 \times 10^{-4}\end{array}$ & $\begin{array}{r}4.5 \times 10^{-4} \\
8.0 \times 10^{-4} \\
7.7 \times 10^{-4} \\
46.0 \times 10^{-4}\end{array}$ & Alteration Reaction \\
\hline $\mathrm{BaTiO}_{3}$ & 0.5 & $\begin{array}{l}23 \\
30 \\
44 \\
45\end{array}$ & $\begin{array}{c}3.6 \times 10^{-4} \\
5.2 \times 10^{-4} \\
5.1 \times 10^{-4} \\
\quad \text { Samp }\end{array}$ & $\begin{array}{r}3.6 \times 10^{-4} \\
10.0 \times 10^{-4} \\
4.9 \times 10^{-4} \\
\text { hattered }\end{array}$ & \\
\hline $\mathrm{CaTi0}$ & 0.7 & $\begin{array}{l}23 \\
30 \\
44 \\
45\end{array}$ & $\begin{array}{l}5.8 \times 10^{-4} \\
6.5 \times 10^{-4} \\
6.7 \times 10^{-4} \\
7.2 \times 10^{-4}\end{array}$ & $\begin{array}{r}5.8 \times 10^{-4} \\
8.9 \times 10^{-4} \\
6.9 \times 10^{-4} \\
32.0 \times 10^{-4}\end{array}$ & Alteration Reaction \\
\hline $\begin{array}{l}\text { Mullite } \\
\left(2 \mathrm{Al}_{2} \mathrm{O}_{3} \cdot \mathrm{SiO}_{2}\right)\end{array}$ & 1.3 & $\begin{array}{r}7 \\
21 \\
42 \\
43\end{array}$ & $\begin{array}{r}0.14 \times 10^{-3} \\
1.6 \times 10^{-3} \\
1.3 \times 10^{-3} \\
1.3 \times 10^{-3}\end{array}$ & $\begin{array}{r}0.14 \times 10^{-3} \\
1.8 \times 10^{-3} \\
1.0 \times 10^{-3} \\
1.0 \times 10^{-3}\end{array}$ & Alteration Reaction \\
\hline
\end{tabular}


TABLE 7. (contd)

\begin{tabular}{|c|c|c|c|c|c|}
\hline \multicolumn{2}{|l|}{ Material } & $\begin{array}{c}\text { Exposure, } \\
\text { days }\end{array}$ & \multicolumn{2}{|c|}{ Average Leach Rate, ${ }^{(b)} \mathrm{g} / \mathrm{cm}^{2}-\mathrm{d}^{(\mathrm{c})}$} & Remarks \\
\hline $\mathrm{ZrSiO}_{4}$ & $i 5$ & $\begin{array}{l}23 \\
30 \\
44 \\
45\end{array}$ & $\begin{array}{l}1.7 \times 10^{-3} \\
1.6 \times 10^{-3} \\
1.1 \times 10^{-3} \\
1.1 \times 10^{-3}\end{array}$ & $\begin{array}{r}1.7 \times 10^{-3} \\
0.68 \times 10^{-4} \\
0.26 \times 10^{-4} \\
0.29 \times 10^{-4}\end{array}$ & \\
\hline $\mathrm{BaZrO}_{3}$ & 1.6 & $\begin{array}{l}23 \\
30 \\
44 \\
45\end{array}$ & $\begin{array}{l}2.0 \times 10^{-3} \\
1.6 \times 10^{-3} \\
1.4 \times 10^{-3} \\
1.4 \times 10^{-3}\end{array}$ & $\begin{array}{r}2.0 \times 10^{-3} \\
0.40 \times 10^{-3} \\
0.84 \times 10^{-3} \\
0.29 \times 10^{-3}\end{array}$ & Alteration Reaction \\
\hline Basalt & $\therefore 6$ & $\begin{array}{l}23 \\
30 \\
44 \\
45\end{array}$ & $\begin{array}{l}4.1 \times 10^{-3} \\
3.6 \times 10^{-3} \\
3.0 \times 10^{-3} \\
3.0 \times 10^{-3}\end{array}$ & $\begin{array}{l}4.1 \times 10^{-3} \\
1.9 \times 10^{-3} \\
1.7 \times 10^{-3} \\
2.5 \times 10^{-3}\end{array}$ & Alteration Reaction \\
\hline Marcor & & $\begin{array}{r}7 \\
21 \\
42 \\
43\end{array}$ & $\begin{array}{r}6.6 \times 10^{-3} \\
5.4 \times 10^{-3} \\
3.5 \times 10^{-3} \\
\text { Samp }\end{array}$ & $\begin{array}{l}\qquad .6 \times 10^{-3} \\
4.8 \times 10^{-3} \\
1.6 \times 10^{-3} \\
\text { hattered }\end{array}$ & Alteration Reaction \\
\hline Pyrocer am 9617 & 7. & $\begin{array}{r}7 \\
21 \\
42 \\
43\end{array}$ & $\begin{array}{r}10.0 \times 10^{-3} \\
9.6 \times 10^{-3} \\
8.2 \times 10^{-3} \\
8.3 \times 10^{-3}\end{array}$ & $\begin{array}{r}10.0 \times 10^{-3} \\
9.2 \times 10^{-3} \\
6.8 \times 10^{-3} \\
9.4 \times 10^{-3}\end{array}$ & Alteration Reaction \\
\hline
\end{tabular}

(a) Tests carried out in a 6.5-L autoclave at a water flow rate of $1.9 \mathrm{~L} / \mathrm{h}$. Geometric surface area of the test specimens varied from 5 to $15 \mathrm{~cm}^{2}$.

(b) Calculated from weight loss measurements.

(c) Based on initial geometric surface area of the specimen.

(d) Average leach rate for exposure time shown.

(e) Average leach rate for specif ic time interval.

graphite and $\mathrm{TiO}_{2}$. In addition, most of the materials suffered localized attack or alteration reactions, which affected the samples to considerable depths. Estimates of the extent of the localized attack or alteration reactions, as determined from photomicrographs of the specimens, are given in Table 8 . The $\mathrm{TiO}_{2}$ specimen developed radial cracks to a considerable depth, but there is evidence that indicates the cracks may have resulted from fabrication problems, rather than attack by the leach solution. Additional discussion of how localized attack or alteration reactions affect the various materials is provided in Section 4.3.

\subsubsection{Static Leach Tests at $150^{\circ} \mathrm{C}$}

The static leach tests at $150^{\circ} \mathrm{C}$ were carried out in sealed gold ampules that were heated in autoclave. Samples of each material were tested with each of the three leach solutions for four different exposure times. A single ceramic sample was used for the four exposures, but the leach solution was replaced after each exposure. The candidate materials were tested in two groups of eight. The testing sequence for one group was 7 days, 14 days, 21 days, and 1 day, and for the second group was 7 days, 14 days, 1 day, and 21 days. After the final tests, the ceramic samples were subjected to ceramographic examination. The change in the 
TABLE 8. Extent of Localized Attack or Alteration Reactions in Ceramic Materials Exposed to Flowing Demineralized Water at $250^{\circ} \mathrm{C}(\mathrm{a})$

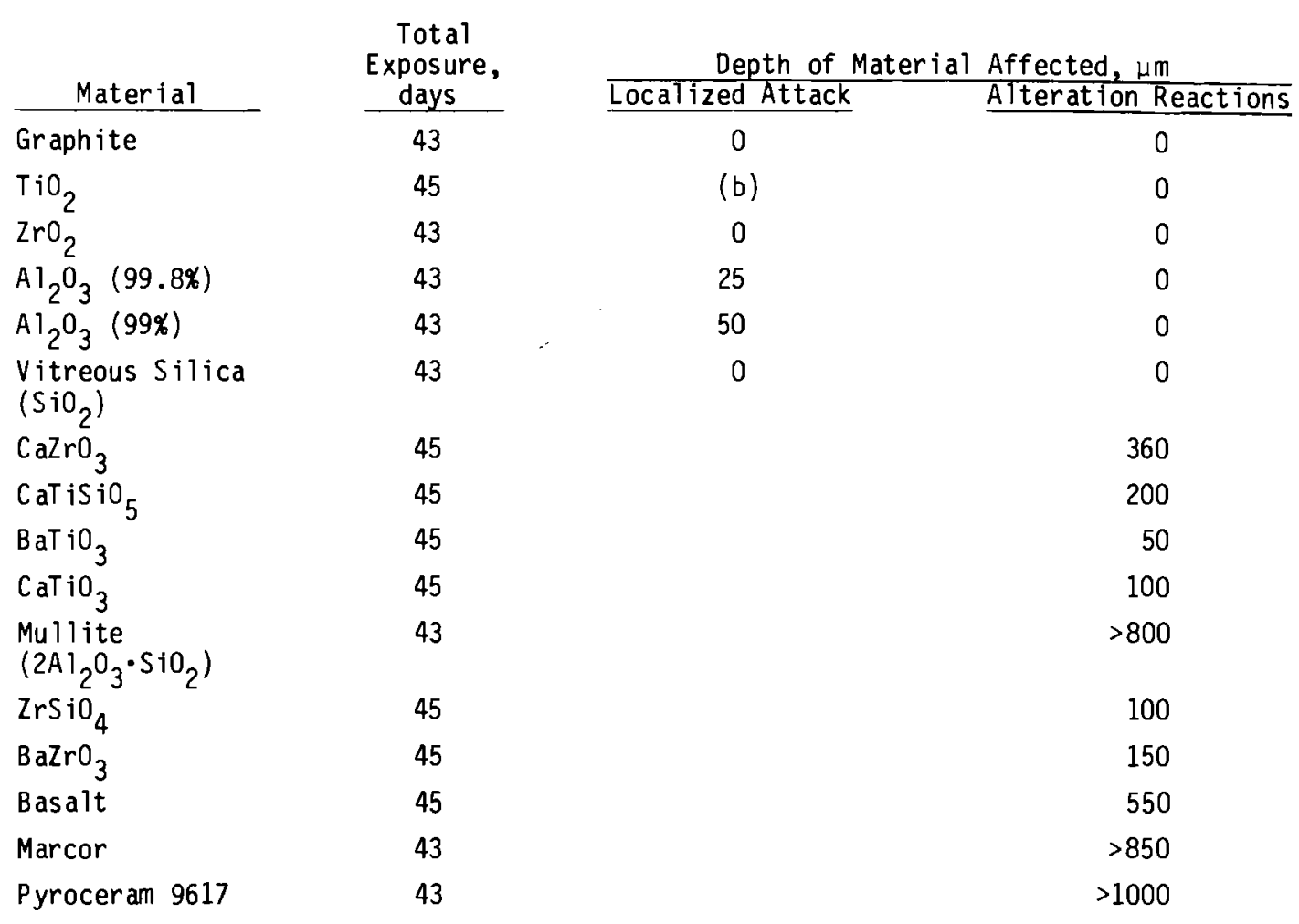

(a) Estimated from photomicrographs.

(b) Specimens exhibited radial cracks to a considerable depth.

testing sequence for the second group of materials was again dictated by autoclave availability and other scheduling problems. The differences in the testing sequences might have some effect on the absolute values of the individual leach rates but should have only minimal effect on the relative leach rates.

The average leach rates for the ceramic materials in the three leach solutions at $150^{\circ} \mathrm{C}$ are given in Tables 9 through 11 . Erratic results were obtained in some tests. Replicate experiments were not performed, and the values given in the tables should only be considered as approximations. The data are adequate, however, for determining the relative leach resistances of the materials tested.

In the tests with demineralized water and Hanford ground water the leach rates, except for graphite, were determined from the weight loss measurements and from the analysis of the leach solutions (graphite leach rates could only be determined by weight loss measurements). In most of the tests, the leach rates calculated from the weight loss measurements were higher than those determined from the solution analyses. The leach rates given in the tables are the 
TABLE 9. Leaching of Ceramic Materials in Demineralized Water at $150^{\circ} \mathrm{C}--$ Static Tests in Sealed Gold Ampules(a)

\begin{tabular}{|c|c|c|c|c|}
\hline Material & $\begin{array}{c}\text { Exposure, } \\
\text { days }\end{array}$ & $\begin{array}{c}\text { Specimen } \\
\text { We ight Loss, } \\
\mathrm{mg} / \mathrm{cm}^{2}(\mathrm{~b}) \\
\end{array}$ & $\begin{array}{c}\text { Average Leach } \\
\text { Rate, }(c) \mathrm{g} / \mathrm{cm}^{2}-\mathrm{d}(b) \\
\end{array}$ & Remarks \\
\hline Graphite & $\begin{array}{r}7 \\
14 \\
21 \\
1\end{array}$ & $\begin{array}{l}0.078 \\
0.055 \\
0.019 \\
0.002\end{array}$ & $\begin{array}{l}1.1 \times 10^{-5} \\
3.9 \times 10^{-6} \\
0.9 \times 10^{-6} \\
2.0 \times 10^{-6}\end{array}$ & \\
\hline $\mathrm{Al}_{2} \mathrm{O}_{3}(99.8 \%)$ & $\begin{array}{r}7 \\
14 \\
21 \\
1\end{array}$ & $\begin{array}{l}0.055 \\
0.046 \\
0.055 \\
0.029\end{array}$ & $\begin{array}{l}7.9 \times 10^{-6} \\
3.2 \times 10^{-6} \\
2.6 \times 10^{-6} \\
2.9 \times 10^{-5}\end{array}$ & \\
\hline $\mathrm{Al}_{2} \mathrm{O}_{3} \quad(99 \%)$ & $\begin{array}{r}7 \\
14 \\
21 \\
1\end{array}$ & $\begin{array}{l}0.10 \\
0.13 \\
0.13 \\
0.045\end{array}$ & $\begin{array}{l}1.4 \times 10^{-5} \\
9.3 \times 10^{-6} \\
6.2 \times 10^{-6} \\
4.5 \times 10^{-5}\end{array}$ & Localized Attack \\
\hline $\begin{array}{l}\text { Mullite } \\
\left(2 \mathrm{Al}_{2} \mathrm{O}_{3} \cdot \mathrm{SiO}_{2}\right)\end{array}$ & $\begin{array}{r}7 \\
14 \\
21 \\
1\end{array}$ & $\begin{array}{l}0.15 \\
0.15 \\
0.15 \\
0.073\end{array}$ & $\begin{array}{l}2.1 \times 10^{-5} \\
1.1 \times 10^{-5} \\
7.2 \times 10^{-6} \\
7.3 \times 10^{-5}\end{array}$ & \\
\hline $\begin{array}{l}\text { Vitreous Silica } \\
\left(\mathrm{SiO}_{2}\right)\end{array}$ & $\begin{array}{r}7 \\
14 \\
21 \\
1\end{array}$ & $\begin{array}{l}0.18 \\
0.23 \\
0.28 \\
0.12\end{array}$ & $\begin{array}{l}2.6 \times 10^{-5} \\
1.6 \times 10^{-5} \\
1.3 \times 10^{-5} \\
1.2 \times 10^{-4}\end{array}$ & \\
\hline $\mathrm{ZrO}_{2}$ & $\begin{array}{r}7 \\
14 \\
21 \\
1\end{array}$ & $\begin{array}{l}0.34 \\
0.30 \\
0.38 \\
0.15\end{array}$ & $\begin{array}{l}4.9 \times 10^{-5} \\
2.1 \times 10^{-5} \\
1.8 \times 10^{-5} \\
1.5 \times 10^{-4}\end{array}$ & \\
\hline Pyroceram 9617 & $\begin{array}{r}7 \\
14 \\
21 \\
1\end{array}$ & $\begin{array}{l}0.27 \\
0.42 \\
0.56 \\
0.19\end{array}$ & $\begin{array}{l}3.9 \times 10^{-5} \\
3.0 \times 10^{-5} \\
2.7 \times 10^{-5} \\
1.9 \times 10^{-4}\end{array}$ & \\
\hline $\mathrm{TiO}_{2}$ & $\begin{array}{r}7 \\
14 \\
1 \\
21\end{array}$ & $\begin{array}{l}0.64 \\
0.52 \\
0.23 \\
0.72\end{array}$ & $\begin{array}{l}9.1 \times 10^{-5} \\
3.7 \times 10^{-5} \\
2.3 \times 10^{-4} \\
3.4 \times 10^{-5}\end{array}$ & \\
\hline CaTiSiO 5 & $\begin{array}{r}7 \\
14 \\
1 \\
21\end{array}$ & $\begin{array}{l}1.18 \\
1.32 \\
1.1 \\
1.28\end{array}$ & $\begin{array}{l}2.6 \times 10^{-4} \\
9.4 \times 10^{-5} \\
1.1 \times 10^{-3} \\
6.1 \times 10^{-5}\end{array}$ & \\
\hline $\mathrm{ZrSiO}_{4}$ & $\begin{array}{r}7 \\
14 \\
1 \\
21\end{array}$ & $\begin{array}{l}0.88 \\
1.24 \\
0.38 \\
2.6\end{array}$ & $\begin{array}{l}1.3 \times 10^{-4} \\
8.9 \times 10^{-5} \\
3.8 \times 10^{-4} \\
1.2 \times 10^{-4}\end{array}$ & \\
\hline $\mathrm{CaTiO}_{3}$ & $\begin{array}{r}7 \\
14 \\
1 \\
21\end{array}$ & $\begin{array}{l}0.84 \\
1.46 \\
0.50 \\
3.1\end{array}$ & $\begin{array}{l}1.2 \times 10^{-4} \\
1.0 \times 10^{-4} \\
5.0 \times 10^{-4} \\
1.5 \times 10^{-4}\end{array}$ & \\
\hline
\end{tabular}


TABLE 9. (contd)

\begin{tabular}{|c|c|c|c|c|}
\hline Material & $\begin{array}{c}\text { Exposure, } \\
\text { days }\end{array}$ & $\begin{array}{c}\text { Specimen } \\
\text { Weight Loss, } \\
\text { mg/cm } 2(b)\end{array}$ & $\begin{array}{c}\text { Average Leach } \\
\text { Rate, }(\mathrm{c})_{\mathrm{g} / \mathrm{cm}^{2}-\mathrm{d}(\mathrm{b})}\end{array}$ & Remarks \\
\hline Basalt & $\begin{array}{r}7 \\
14 \\
1 \\
21\end{array}$ & $\begin{array}{l}2.7 \\
2.2 \\
1.5 \\
2.6\end{array}$ & $\begin{array}{l}3.9 \times 10^{-4} \\
1.6 \times 10^{-4} \\
1.5 \times 10^{-3} \\
1.2 \times 10^{-4}\end{array}$ & \\
\hline Marcor & $\begin{array}{r}7 \\
14 \\
21 \\
1\end{array}$ & $\begin{array}{l}1.8 \\
2.9 \\
2.2 \\
1.3\end{array}$ & $\begin{array}{l}2.6 \times 10^{-4} \\
2.1 \times 10^{-4} \\
1.0 \times 10^{-4} \\
1.3 \times 10^{-3}\end{array}$ & \\
\hline $\mathrm{CaZrO}_{3}$ & $\begin{array}{r}7 \\
14 \\
1 \\
21\end{array}$ & $\begin{array}{l}0.19 \\
4.6 \\
2.9 \\
5.8\end{array}$ & $\begin{array}{l}2.7 \times 10^{-5} \\
3.3 \times 10^{-4} \\
2.9 \times 10^{-3} \\
2.8 \times 10^{-4}\end{array}$ & Alteration Reaction \\
\hline $\mathrm{BaZrO}_{3}$ & $\begin{array}{r}7 \\
14 \\
1 \\
21\end{array}$ & $\begin{array}{r}9.8 \\
7.3 \\
4.1 \\
12\end{array}$ & $\begin{array}{l}1.4 \times 10^{-3} \\
5.2 \times 10^{-4} \\
4.1 \times 10^{-3} \\
5.7 \times 10^{-3}\end{array}$ & Sample Cracked \\
\hline $\mathrm{BaTiO}_{3}$ & $\begin{array}{r}7 \\
14 \\
1 \\
21\end{array}$ & Samp & $\begin{array}{l}2.0 \times 10^{-3} \\
4.9 \times 10^{-3} \\
8.0 \times 10^{-3} \\
\text { Shattered }\end{array}$ & \\
\hline
\end{tabular}

(a) $S / V \cong 0.4 \mathrm{~cm}^{-1}$ based on geometric surface area of specimen.

(b) Based on geometric surface area of specimen.

(c) Average leach rate for exposure time shown.

larger of the values determined by the two methods. In general, there was better agreement in the two sets of values for materials exhibiting moderate and high leach rates than for materials having very low leach rates (i.e., $\mathrm{Al}_{2} \mathrm{O}_{3}, \mathrm{TiO}_{2}, \mathrm{ZrO}_{2}$ ).

In the tests with the brine solution, the leach rates could only be determined from the weight loss measurements. Solution analyses could not be used because the brine initially contained large concentrations of cations that were present in the ceramic materials, either as primary constituents or impurities, and which leached from the materials during testing.

In almost all of the static tests at $150^{\circ} \mathrm{C}$ the leach rate decreased with increasing exposure time. This is to be expected in static tests where the leach solution can become saturated with the dissolved species, especially in the tests under consideration where the solution volume is small and the S/V ratio high. Analysis of the leach solutions indicated that saturation effects did serve to control the leach rates of most of the ceramic materials tested at $150^{\circ} \mathrm{C}$.

Most of the materials tested exhibited a higher leach rate in the demineralized water than in the other two solutions. The data indicate that this is due in part because the saturation concentrations of most of the dissolved species appear to be higher in the demineralized water than in groundwater and brine solutions. 
TABLE 10. Leaching of Ceramic Materials in Hanford Ground Water at $150^{\circ} \mathrm{C}--$ Static Tests in Sealed Gold Ampules(a)

\begin{tabular}{|c|c|c|c|c|}
\hline Material & $\begin{array}{c}\text { Exposure, } \\
\text { days }\end{array}$ & $\begin{array}{c}\text { Spec imen } \\
\text { Weight Loss, } \\
\mathrm{mg} / \mathrm{cm}^{2}(\mathrm{~b}) \\
\end{array}$ & $\begin{array}{r}\text { Aver age Leach } \\
\text { Rate, (c) } \mathrm{g} / \mathrm{cm}^{2}-d^{(b)}\end{array}$ & Remarks \\
\hline $\mathrm{Al}_{2} \mathrm{O}_{3} \quad(99.8 \%)$ & $\begin{array}{r}7 \\
14 \\
21 \\
1\end{array}$ & $\begin{array}{l}0.048 \\
0.034 \\
0.058 \\
0.016\end{array}$ & $\begin{array}{l}6.9 \times 10^{-6} \\
2.4 \times 10^{-6} \\
2.8 \times 10^{-6} \\
1.6 \times 10^{-5}\end{array}$ & \\
\hline Graphite & $\begin{array}{r}7 \\
14 \\
21 \\
1\end{array}$ & $\begin{array}{l}0.086 \\
0.074 \\
0.075 \\
0.063\end{array}$ & $\begin{array}{l}1.2 \times 10^{-5} \\
5.3 \times 10^{-6} \\
3.6 \times 10^{-6} \\
6.3 \times 10^{-5}\end{array}$ & \\
\hline $\mathrm{Al}_{2} \mathrm{O}_{3} \quad$ (99\%) & $\begin{array}{r}7 \\
14 \\
21 \\
1\end{array}$ & $\begin{array}{l}0.15 \\
0.094 \\
0.13 \\
0.062\end{array}$ & $\begin{array}{l}2.1 \times 10^{-5} \\
6.7 \times 10^{-6} \\
6.2 \times 10^{-6} \\
6.2 \times 10^{-5}\end{array}$ & \\
\hline $\begin{array}{l}\text { Mullite } \\
\left(2 \mathrm{Al}_{2} \mathrm{O}_{3} \cdot \mathrm{SiO}_{2}\right)\end{array}$ & $\begin{array}{r}7 \\
14 \\
21 \\
1\end{array}$ & $\begin{array}{l}0.12 \\
0.17 \\
0.18 \\
0.11\end{array}$ & $\begin{array}{l}1.7 \times 10^{-5} \\
1.2 \times 10^{-5} \\
8.6 \times 10^{-6} \\
1.1 \times 10^{-4}\end{array}$ & \\
\hline $\mathrm{ZrO}_{2}$ & $\begin{array}{r}7 \\
14 \\
21 \\
1\end{array}$ & $\begin{array}{l}0.20 \\
0.20 \\
0.25 \\
0.093\end{array}$ & $\begin{array}{l}2.9 \times 10^{-5} \\
1.4 \times 10^{-5} \\
1.2 \times 10^{-5} \\
9.3 \times 10^{-5}\end{array}$ & \\
\hline Pyroceram 9617 & $\begin{array}{r}7 \\
14 \\
21 \\
1\end{array}$ & $\begin{array}{l}0.15 \\
0.19 \\
0.25 \\
0.16\end{array}$ & $\begin{array}{l}2.1 \times 10^{-5} \\
1.4 \times 10^{-5} \\
1.2 \times 10^{-5} \\
1.6 \times 10^{-4}\end{array}$ & $\begin{array}{l}\text { Alteration Reaction } \\
\text { Specimen Cracked }\end{array}$ \\
\hline $\mathrm{CaZrO}_{3}$ & $\begin{array}{r}7 \\
14 \\
1 \\
21\end{array}$ & $\begin{array}{l}0.45 \\
0.62 \\
0.43 \\
0.45\end{array}$ & $\begin{array}{l}6.4 \times 10^{-5} \\
4.4 \times 10^{-5} \\
4.3 \times 10^{-4} \\
2.1 \times 10^{-5}\end{array}$ & \\
\hline $\mathrm{ZrSiO}_{4}$ & $\begin{array}{r}7 \\
14 \\
1 \\
21\end{array}$ & $\begin{array}{l}0.86 \\
0.65 \\
0.16 \\
0.88\end{array}$ & $\begin{array}{l}1.2 \times 10^{-4} \\
4.0 \times 10^{-5} \\
1.6 \times 10^{-4} \\
4.2 \times 10^{-5}\end{array}$ & \\
\hline $\mathrm{CaTi0}$ & $\begin{array}{r}7 \\
14 \\
1 \\
21\end{array}$ & $\begin{array}{l}1.15 \\
0.96 \\
0.5 \\
1.7\end{array}$ & $\begin{array}{l}1.6 \times 10^{-4} \\
6.9 \times 10^{-5} \\
5.0 \times 10^{-4} \\
8.1 \times 10^{-5}\end{array}$ & \\
\hline $\mathrm{TiO}_{2}$ & $\begin{array}{r}7 \\
14 \\
1 \\
21\end{array}$ & $\begin{array}{l}1.1 \\
0.56 \\
0.8 \\
1.3\end{array}$ & $\begin{array}{l}1.7 \times 10^{-4} \\
4.0 \times 10^{-5} \\
8.0 \times 10^{-4} \\
6.2 \times 10^{-5}\end{array}$ & \\
\hline $\mathrm{CaTiSiO}_{5}$ & $\begin{array}{r}7 \\
14 \\
1 \\
21\end{array}$ & $\begin{array}{l}1.0 \\
1.3 \\
0.8 \\
1.8\end{array}$ & $\begin{array}{l}1.4 \times 10^{-4} \\
9.3 \times 10^{-5} \\
8.0 \times 10^{-4} \\
8.6 \times 10^{-5}\end{array}$ & \\
\hline
\end{tabular}


TABLE 10. (contd)

\begin{tabular}{|c|c|c|c|c|}
\hline Material & $\begin{array}{c}\text { Exposure, } \\
\text { days }\end{array}$ & $\begin{array}{c}\text { Spec imen } \\
\text { We ight } L p s s, \\
\mathrm{mg} / \mathrm{cm}^{2}(\mathrm{~b}) \\
\end{array}$ & $\begin{array}{r}\text { Aver age Leach } \\
\text { Rate, }(c){ }_{\mathrm{g} / \mathrm{cm}^{2}-\mathrm{d}}(\mathrm{b}) \\
\end{array}$ & Remarks \\
\hline $\begin{array}{l}\text { Vitreous Silica } \\
\left(\mathrm{SiO}_{2}\right)\end{array}$ & $\begin{array}{r}7 \\
14 \\
21 \\
1\end{array}$ & $\begin{array}{l}1.7 \\
1.5 \\
1.9 \\
1.3\end{array}$ & $\begin{array}{l}2.4 \times 10^{-4} \\
1.1 \times 10^{-4} \\
9.0 \times 10^{-5} \\
1.3 \times 10^{-3}\end{array}$ & \\
\hline $\mathrm{BaTiO}_{3}$ & $\begin{array}{r}7 \\
14 \\
1 \\
21\end{array}$ & $\begin{array}{l}2.2 \\
3.6 \\
0.3 \\
4.3\end{array}$ & $\begin{array}{l}3.1 \times 10^{-4} \\
2.6 \times 10^{-4} \\
3.0 \times 10^{-4} \\
2.0 \times 10^{-4}\end{array}$ & \\
\hline Basalt & $\begin{array}{r}7 \\
14 \\
1 \\
21\end{array}$ & $\begin{array}{l}2.9 \\
3.8 \\
1.5 \\
3.6\end{array}$ & $\begin{array}{l}4.1 \times 10^{-4} \\
2.7 \times 10^{-4} \\
1.5 \times 10^{-3} \\
1.7 \times 10^{-4}\end{array}$ & \\
\hline Marcor & $\begin{array}{r}7 \\
14 \\
21 \\
1\end{array}$ & $\begin{array}{l}2.8 \\
4.4 \\
3.9 \\
1.7\end{array}$ & $\begin{array}{l}4.0 \times 10^{-4} \\
3.1 \times 10^{-4} \\
1.9 \times 10^{-4} \\
1.7 \times 10^{-3}\end{array}$ & \\
\hline $\mathrm{BaZrO}_{3}$ & $\begin{array}{r}7 \\
14 \\
1 \\
21\end{array}$ & $\begin{array}{l}16 \\
4.4 \\
1.5 \\
12\end{array}$ & $\begin{array}{l}2.3 \times 10^{-3} \\
3.1 \times 10^{-4} \\
1.5 \times 10^{-3} \\
5.7 \times 10^{-4}\end{array}$ & \\
\hline
\end{tabular}

(a) $S / V \cong 0.4 \mathrm{~cm}^{-1}$ based on geometric surface area of the specimen.

(b) Based on geometric surface area of specimen.

(c) Average leach rate for exposure time shown.

Localized attack or alteration reactions did not appear to be a major problem with most of the materials tested at $150^{\circ} \mathrm{C}$. A few specimens did exhibit a powdery surface after testing, and some developed cracks. Additional information on the subject is provided in Section 4.3 , where the test materials are discussed individually.

\subsubsection{Static Leach Tests at $250^{\circ} \mathrm{C}$}

The static leach tests at $250^{\circ} \mathrm{C}$ were also carried out in gold ampules inside an autoclave. Test procedures were identical to those used in the $150^{\circ} \mathrm{C}$ static tests. The materials were tested in two groups of eight: the testing sequence for one group was 7 days, 14 days, 21 days, and 1 day, and for the second group was 23 days, 7 days, 14 days, and 1 day. After the final tests, the ceramic specimens were subjected to ceramographic examination. The change in the testing sequence for the second group of materials was again dictated by autoclave availability and other scheduling problems.

The average leach rates measured at $250^{\circ} \mathrm{C}$ in the three leach solutions are given in Tables 12 through 14 . As was the case with the $150^{\circ} \mathrm{C}$ tests, erratic results were obtained in several of the $250^{\circ} \mathrm{C}$ tests. Leach rates in the demineralized water and ground water were determined from weight loss measurements and from analys is of the leach solutions--except for 
TABLE 11. Leaching of Ceramic Materials in Brine at $150^{\circ} \mathrm{C}--$ Static Tests in Sealed Gold

\begin{tabular}{|c|c|c|c|c|}
\hline Material & $\begin{array}{c}\text { Exposure, } \\
\text { days }\end{array}$ & $\begin{array}{c}\text { Specimen } \\
\text { Weight Loss, } \\
\text { mg/cm2 } 2 \text { b) }\end{array}$ & $\begin{array}{r}\text { Aver age Leach } \\
\text { Rate, }(c) \mathrm{g} / \mathrm{cm}^{2}-\mathrm{d}(\mathrm{b})\end{array}$ & Remarks \\
\hline Graphite & $\begin{array}{r}7 \\
14 \\
21 \\
1\end{array}$ & $\begin{array}{l}\text { wt. gain } \\
\text { wt. gain } \\
\text { wt. gain } \\
\text { wt. gain }\end{array}$ & $\begin{array}{l}--- \\
--- \\
--- \\
---\end{array}$ & \\
\hline $\mathrm{TiO}_{2}$ & $\begin{array}{r}7 \\
14 \\
1 \\
21\end{array}$ & $\begin{array}{l}\text { wt. gain } \\
\text { wt. gain } \\
\text { wt. gain } \\
\text { wt. gain }\end{array}$ & $\begin{array}{l}--- \\
--- \\
--- \\
---\end{array}$ & \\
\hline $\mathrm{Al}_{2} \mathrm{O}_{3} \quad(99.8 \%)$ & $\begin{array}{r}7 \\
14 \\
21 \\
1\end{array}$ & $\begin{array}{l}0.018 \\
0.018 \\
0.028 \\
0.009\end{array}$ & $\begin{array}{l}2.6 \times 10^{-6} \\
1.3 \times 10^{-6} \\
1.3 \times 10^{-6} \\
9.0 \times 10^{-6}\end{array}$ & \\
\hline $\mathrm{Al}_{2} \mathrm{O}_{3} \quad(99 \%)$ & $\begin{array}{r}7 \\
14 \\
21 \\
1\end{array}$ & $\begin{array}{l}0.077 \\
0.046 \\
0.050 \\
0.038\end{array}$ & $\begin{array}{l}1.1 \times 10^{-5} \\
6.6 \times 10^{-6} \\
2.4 \times 10^{-6} \\
3.8 \times 10^{-3}\end{array}$ & \\
\hline $\begin{array}{l}\text { Mullite } \\
\left(2 \mathrm{Al}_{2} \mathrm{O}_{3} \cdot \mathrm{SiO}_{2}\right)\end{array}$ & $\begin{array}{r}7 \\
14 \\
21 \\
1\end{array}$ & $\begin{array}{l}0.082 \\
0.060 \\
0.095 \\
0.057\end{array}$ & $\begin{array}{l}1.2 \times 10^{-5} \\
4.3 \times 10^{-6} \\
4.5 \times 10^{-6} \\
5.7 \times 10^{-3}\end{array}$ & \\
\hline $\begin{array}{l}\text { Vitreous Silica } \\
\left(\mathrm{SiO}_{2}\right)\end{array}$ & $\begin{array}{r}7 \\
14 \\
21 \\
1\end{array}$ & $\begin{array}{l}0.12 \\
0.047 \\
0.095 \\
0.086\end{array}$ & $\begin{array}{l}1.7 \times 10^{-5} \\
4.5 \times 10^{-6} \\
2.6 \times 10^{-6} \\
8.6 \times 10^{-5}\end{array}$ & \\
\hline Pyroceram 9617 & $\begin{array}{r}7 \\
14 \\
21 \\
1\end{array}$ & $\begin{array}{l}0.071 \\
0.10 \\
0.19 \\
0.061\end{array}$ & $\begin{array}{l}1.0 \times 10^{-5} \\
7.1 \times 10^{-6} \\
9.0 \times 10^{-6} \\
6.1 \times 10^{-5}\end{array}$ & \\
\hline $\mathrm{ZrO}_{2}$ & $\begin{array}{r}7 \\
14 \\
21 \\
1\end{array}$ & $\begin{array}{l}0.010 \\
0.070 \\
0.28 \\
0.041\end{array}$ & $\begin{array}{l}1.4 \times 10^{-6} \\
5.0 \times 10^{-6} \\
1.3 \times 10^{-5} \\
4.1 \times 10^{-5}\end{array}$ & \\
\hline $\mathrm{CaTiO} \mathrm{O}_{3}$ & $\begin{array}{r}7 \\
14 \\
1 \\
21\end{array}$ & $\begin{array}{l}0.92 \\
0.80 \\
0.5 \\
5.9\end{array}$ & $\begin{array}{l}1.3 \times 10^{-4} \\
5.7 \times 10^{-5} \\
5.0 \times 10^{-4} \\
2.8 \times 10^{-4}\end{array}$ & \\
\hline $\mathrm{ZrSiO}_{4}$ & $\begin{array}{r}7 \\
14 \\
1 \\
21\end{array}$ & $\begin{array}{l}1.5 \\
3.6 \\
2.3 \\
4.5\end{array}$ & $\begin{array}{l}2.1 \times 10^{-4} \\
2.6 \times 10^{-4} \\
2.3 \times 10^{-3} \\
2.1 \times 10^{-4}\end{array}$ & Alteration Reaction \\
\hline Marcor & $\begin{array}{r}7 \\
14 \\
21 \\
1\end{array}$ & $\begin{array}{l}2.4 \\
3.8 \\
4.4 \\
1.5\end{array}$ & $\begin{array}{l}3.4 \times 10^{-4} \\
2.7 \times 10^{-4} \\
2.1 \times 10^{-4} \\
1.5 \times 10^{-3}\end{array}$ & \\
\hline
\end{tabular}


TABLE 11. (contd)

\begin{tabular}{|c|c|c|c|c|}
\hline Material & $\begin{array}{c}\text { Exposure, } \\
\text { days }\end{array}$ & $\begin{array}{c}\text { Specimen } \\
\text { Weight Loss, } \\
\mathrm{mg} / \mathrm{cm}^{2}(\mathrm{~b})\end{array}$ & $\begin{array}{l}\text { Aver age Leach } \\
\text { Rate, (c) } \mathrm{g} / \mathrm{cm}^{2}-\mathrm{d}(\mathrm{b})\end{array}$ & Remarks \\
\hline $\mathrm{BaTi0}_{3}$ & $\begin{array}{r}7 \\
14 \\
1 \\
21\end{array}$ & $\begin{array}{l}16 \\
8.6 \\
1.3 \\
17\end{array}$ & $\begin{array}{l}2.3 \times 10^{-3} \\
6.1 \times 10^{-4} \\
1.3 \times 10^{-3} \\
8.1 \times 10^{-4}\end{array}$ & \\
\hline $\mathrm{BaZrO}_{3}$ & $\begin{array}{r}7 \\
14 \\
1 \\
21\end{array}$ & $\begin{array}{l}0.9 \\
6.2 \\
9.9\end{array}$ & $\begin{array}{r}1.3 \times 10^{-4} \\
4.4 \times 10^{-4} \\
9.9 \times 10^{-3} \\
\text { Shattered }\end{array}$ & Alteration Reaction \\
\hline
\end{tabular}

(a) $S / V \cong 0.4 \mathrm{~cm}^{-1}$ based on geometric surface area of the specimen.

(b) Based on geometric surface area of specimen.

(c) Average leach rate for exposure time shown.

graphite, whose leach rates were determined only from the weight loss measurements. Leach rates in the brine solution were determined only from the weight loss measurements. Where there was a lack of agreement between the leach rates determined by the two methods, the higher values are reported in the tables.

Unlike the results obtained at $150^{\circ} \mathrm{C}$, in many of the $250^{\circ} \mathrm{C}$ tests the leach rates determined from the solution analyses were higher than those calculated from the weight loss measurements. This was because several of the ceramic materials tested at $250^{\circ} \mathrm{C}$ suffered alteration reactions that affected the weight loss measurements. In several of the tests the specimens cracked or shattered into two or more pieces during testing, which invalidated the weight loss measurements.

In most of the static tests at $250^{\circ} \mathrm{C}$ the leach rates decreased with increasing exposure times because of saturation effects. In general, leach rates were higher in demineralized water than in the groundwater or brine solution. Except for two materials, graphite and $\mathrm{TiO}_{2}$, the leach rates in flowing demineralized water at $250^{\circ} \mathrm{C}$ were much higher than were the rates measured in the static demineralized water tests at $250^{\circ} \mathrm{C}$. This also illustrates the effect saturation limits can have on the leach rates of slightly soluble materials in static systems. It was not determined why the leach rate of $\mathrm{TiO}_{2}$ was lower in the flowing demineralized water at $250^{\circ} \mathrm{C}$ than in the static test at $250^{\circ} \mathrm{C}$. The higher leach rate of graphite in the static test at $250^{\circ} \mathrm{C}$ as compared to the dynamic test can probably be explained by the reaction of the graphite with dissolved oxygen, since the gold ampule was only partially filled with demineralized water and the remaining space with air (see Section 4.3.1).

As expected, the leach rates determined in the static tests at $250^{\circ} \mathrm{C}$ were generally higher than the corresponding rates measured at $150^{\circ} \mathrm{C}$. Because of the erratic nature of some of the data, however, some anomalies were apparent. The increase in leach rates with temperature was not as great as one might anticipate. On an overall basis, the leach rates at $250^{\circ} \mathrm{C}$ were about three times higher than the rates at $150^{\circ} \mathrm{C}$, although much greater increases were observed in some cases. 
TABLE 12. Leaching of Ceramic Materials in Demineralized Water at $250^{\circ} \mathrm{C}--$ Static Tests in Sealed Gold Ampules(a)

\begin{tabular}{|c|c|c|c|c|}
\hline Material & $\begin{array}{c}\text { Exposure, } \\
\text { days } \\
\end{array}$ & $\begin{array}{c}\text { Specimen } \\
\text { Weight Loss, } \\
\mathrm{mg} / \mathrm{cm}^{2}(\mathrm{~b}) \\
\end{array}$ & $\begin{array}{r}\text { Average Leach } \\
\text { Rate, }(c) \mathrm{g} / \mathrm{cm}^{2}-\mathrm{d}(\mathrm{b}) \\
\end{array}$ & Remarks \\
\hline $\mathrm{Al}_{2} \mathrm{O}_{3} \quad(99.8 \%)$ & $\begin{array}{r}7 \\
14 \\
21 \\
1\end{array}$ & $\begin{array}{l}0.092 \\
0.12 \\
0.11 \\
0.04\end{array}$ & $\begin{array}{l}1.3 \times 10^{-5} \\
8.6 \times 10^{-6} \\
5.2 \times 10^{-6} \\
4.0 \times 10^{-5}\end{array}$ & Localized Attack \\
\hline Graphite & $\begin{array}{r}7 \\
14 \\
21 \\
1\end{array}$ & $\begin{array}{l}0.21 \\
0.16 \\
0.16 \\
0.054\end{array}$ & $\begin{array}{l}3.0 \times 10^{-5} \\
1.1 \times 10^{-5} \\
7.6 \times 10^{-6} \\
5.4 \times 10^{-5}\end{array}$ & \\
\hline $\mathrm{Al}_{2} \mathrm{O}_{3} \quad(99 \%)$ & $\begin{array}{r}7 \\
14 \\
21 \\
1\end{array}$ & $\begin{array}{l}0.30 \\
0.34 \\
0.44 \\
0.082\end{array}$ & $\begin{array}{l}4.3 \times 10^{-5} \\
2.4 \times 10^{-5} \\
2.1 \times 10^{-5} \\
8.2 \times 10^{-5}\end{array}$ & Localized Attack \\
\hline $\mathrm{TiO}_{2}$ & $\begin{array}{r}23 \\
7 \\
14 \\
1\end{array}$ & $\begin{array}{l}0.38 \\
0.36 \\
0.64 \\
0.27\end{array}$ & $\begin{array}{l}1.7 \times 10^{-5} \\
5.1 \times 10^{-5} \\
4.6 \times 10^{-5} \\
2.7 \times 10^{-4}\end{array}$ & \\
\hline $\mathrm{CaTiO}_{3}$ & $\begin{array}{r}23 \\
7 \\
14 \\
1\end{array}$ & $\begin{array}{l}0.24 \\
0.066 \\
0.14 \\
1.3\end{array}$ & $\begin{array}{l}1.0 \times 10^{-5} \\
9.4 \times 10^{-6} \\
1.0 \times 10^{-5} \\
1.3 \times 10^{-3}\end{array}$ & Specimen Cracked \\
\hline $\mathrm{ZrO}_{2}$ & $\begin{array}{r}7 \\
14 \\
21 \\
1\end{array}$ & $\begin{array}{l}0.43 \\
0.49 \\
0.55 \\
0.16\end{array}$ & $\begin{array}{l}6.1 \times 10^{-5} \\
3.5 \times 10^{-5} \\
2.6 \times 10^{-5} \\
1.6 \times 10^{-4}\end{array}$ & \\
\hline $\begin{array}{l}\text { Vitreous Silica } \\
\left(\mathrm{SiO}_{2}\right)\end{array}$ & $\begin{array}{r}7 \\
14 \\
21 \\
1\end{array}$ & $\begin{array}{l}1.6 \\
1.7 \\
1.9 \\
0.20\end{array}$ & $\begin{array}{l}2.3 \times 10^{-4} \\
1.2 \times 10^{-4} \\
9.0 \times 10^{-5} \\
2.0 \times 10^{-4}\end{array}$ & \\
\hline Basalt & $\begin{array}{r}23 \\
7 \\
14 \\
1\end{array}$ & $\begin{array}{l}4.0 \\
3.9 \\
3.7 \\
0.55\end{array}$ & $\begin{array}{l}1.7 \times 10^{-4} \\
5.6 \times 10^{-4} \\
2.6 \times 10^{-4} \\
5.5 \times 10^{-4}\end{array}$ & Alteration Reaction \\
\hline $\mathrm{CaTiSiO} 5$ & $\begin{array}{r}23 \\
7 \\
14 \\
1\end{array}$ & $\begin{array}{l}0.75 \\
1.1 \\
6.4 \\
1.3\end{array}$ & $\begin{array}{l}2.4 \times 10^{-5} \\
1.6 \times 10^{-4} \\
4.6 \times 10^{-4} \\
1.3 \times 10^{-3}\end{array}$ & Alteration Reaction \\
\hline $\begin{array}{l}\text { Mullite } \\
\left(2 \mathrm{Al}_{2} \mathrm{O}_{3} \cdot \mathrm{SiO}_{2}\right)\end{array}$ & $\begin{array}{r}7 \\
14 \\
21 \\
1\end{array}$ & $\begin{array}{l}2.3 \\
3.8 \\
3.6 \\
1.4\end{array}$ & $\begin{array}{l}3.3 \times 10^{-4} \\
2.7 \times 10^{-4} \\
1.7 \times 10^{-4} \\
1.4 \times 10^{-3}\end{array}$ & Alteration Reaction \\
\hline $\mathrm{CaZrO}_{3}$ & $\begin{array}{r}23 \\
7 \\
14 \\
1\end{array}$ & $\begin{array}{l}3.3 \\
2.4 \\
5.5 \\
3.8\end{array}$ & $\begin{array}{l}1.4 \times 10^{-4} \\
3.4 \times 10^{-4} \\
3.9 \times 10^{-4} \\
3.8 \times 10^{-3}\end{array}$ & Alteration Reaction \\
\hline
\end{tabular}


TABLE 12. (contd)

\begin{tabular}{|c|c|c|c|c|}
\hline Material & $\begin{array}{c}\text { Exposure, } \\
\text { days }\end{array}$ & $\begin{array}{l}\text { Specimen } \\
\text { Weight Loss, } \\
\mathrm{mg} / \mathrm{cm}^{2}(\mathrm{~b}) \\
\end{array}$ & $\begin{array}{c}\text { Aver age Leach } \\
\text { Rate, }(c)_{\mathrm{g} / \mathrm{cm}^{2}-\mathrm{d}}(\mathrm{b})\end{array}$ & Remarks \\
\hline $\mathrm{ZrSiO}_{4}$ & $\begin{array}{r}23 \\
7 \\
14 \\
1\end{array}$ & $\begin{array}{ll}2.9 & \\
4.8 & \\
7.3 & \\
& \text { San }\end{array}$ & $\begin{array}{r}1.3 \times 10^{-4} \\
6.9 \times 10^{-4} \\
5.2 \times 10^{-4} \\
\text { Shattered }\end{array}$ & \\
\hline Pyrocer am 9617 & $\begin{array}{r}7 \\
14 \\
21 \\
1\end{array}$ & $\begin{array}{c}4.7 \\
5.6 \\
13 \\
4.1\end{array}$ & $\begin{array}{l}6.7 \times 10^{-4} \\
4.0 \times 10^{-4} \\
6.2 \times 10^{-4} \\
4.1 \times 10^{-3}\end{array}$ & $\begin{array}{l}\text { Alteration Reaction } \\
\text { Sample Cracked }\end{array}$ \\
\hline $\mathrm{BaZrO}_{3}$ & $\begin{array}{r}23 \\
7 \\
14 \\
1\end{array}$ & $\begin{array}{l}5.8 \\
7.3 \\
16 \\
28\end{array}$ & $\begin{array}{l}2.5 \times 10^{-4} \\
1.0 \times 10^{-3} \\
1.1 \times 10^{-3} \\
2.8 \times 10^{-2}\end{array}$ & $\begin{array}{l}\text { Alteration Reaction } \\
\text { Sample Cracked }\end{array}$ \\
\hline $\mathrm{BaTiO}_{3}$ & $\begin{array}{r}23 \\
7 \\
14 \\
1\end{array}$ & $\begin{array}{l}1.0 \\
4.8 \\
23\end{array}$ & $\begin{array}{r}4.0 \times 10^{-5} \\
6.9 \times 10^{-4} \\
1.6 \times 10^{-3} \\
\text { Shattered }\end{array}$ & \\
\hline Marcor & $\begin{array}{r}7 \\
14 \\
21 \\
1\end{array}$ & $\begin{array}{l}17 \\
23 \\
28 \\
12\end{array}$ & $\begin{array}{l}2.4 \times 10^{-3} \\
1.6 \times 10^{-3} \\
1.3 \times 10^{-3} \\
1.2 \times 10^{2}\end{array}$ & $\begin{array}{l}\text { Alteration Reaction } \\
\text { Spec imen Cracked }\end{array}$ \\
\hline
\end{tabular}

(a) $S / V \cong 0.4 \mathrm{~cm}^{-1}$ based on geometric surface area of the specimen.

(b) Based on geometric surface area of specimen.

(c) Average leach rate for exposure time shown.

\subsubsection{Relative Leach Rates}

It is difficult to make precise rankings of the candidate materials based on the leach rate data obtained in the various tests. In most of the tests the leach rates of the ceramic materials varied with time. Additional long-term leaching tests are needed in order to make quantitative comparisons of the candidates. In some of the tests erratic results were obtained, and replicate experiments are needed in order to ensure the reliability of the leach rate data. Unfortunately, it was not possible to carry out the additional testing needed to allow quantitative ranking of the candidate materials for each leach test. It was possible, however, to make some qualitative comparisons of the materials tested based on the leach rate data obtained in the various tests. This has been done, and in Table 15 the candidate materials are listed in order of their estimated leach resistance for each of the leach tests. It must be remembered that the rankings given in Table 15 are not absolute. The leach resistance of any given materials should definitely be greater than that of a material several positions lower on the scale. In the case of adjacent materials, however, it is impossible in most cases to say that one is more leach-resistant than the other.

A cursory examination of Table 15 shows that five materials--namely graphite, $\mathrm{TiO}_{2}, \mathrm{ZrO}_{2}$, and the two grades of $\mathrm{Al}_{2} \mathrm{O}_{3}$--exhibit the highest leach resistance in most of the tests. Some 
TABLE 13. Leaching of Ceramic Materials in Hanford Ground Water at $250^{\circ} \mathrm{C}-$-Static Test in Sealed Gold Ampules(a)

\begin{tabular}{|c|c|c|c|c|c|}
\hline Material & & $\begin{array}{c}\text { Exposure, } \\
\text { days }\end{array}$ & $\begin{array}{c}\text { Spec imen } \\
\text { Weight Loss, } \\
\mathrm{mg} / \mathrm{cm}^{2}(\mathrm{~b}) \\
\end{array}$ & $\begin{array}{r}\text { Aver age Leach } \\
\text { Rate, }(c)^{\mathrm{g} / \mathrm{cm}^{2}-\mathrm{d}(b)}\end{array}$ & Remarks \\
\hline $\mathrm{Al}_{2} \mathrm{O}_{3}(99.8 \%)$ & .001 & $\begin{array}{r}7 \\
14 \\
21 \\
1\end{array}$ & $\begin{array}{l}0.14 \\
0.09 \\
0.15 \\
0.03\end{array}$ & $\begin{array}{l}2.0 \times 10^{-5} \\
6.4 \times 10^{-6} \\
7.0 \times 10^{-6} \\
3.0 \times 10^{-5}\end{array}$ & \\
\hline Graphite & 13 & $\begin{array}{r}7 \\
14 \\
21 \\
1\end{array}$ & $\begin{array}{l}0.21 \\
\quad \text { Leach } \\
0.15 \\
0.046\end{array}$ & $\begin{array}{r}3.0 \times 10^{-5} \\
\text { Solution Lost } \\
7.1 \times 10^{-6} \\
4.6 \times 10^{-5}\end{array}$ & \\
\hline $\mathrm{ZrO}_{2}$ & $+c$ & $\begin{array}{r}7 \\
14 \\
21 \\
1\end{array}$ & $\begin{array}{l}0.094 \\
0.17 \\
0.36 \\
0.029\end{array}$ & $\begin{array}{l}1.3 \times 10^{-5} \\
1.2 \times 10^{-5} \\
1.7 \times 10^{-5} \\
2.9 \times 10^{-5}\end{array}$ & \\
\hline $\mathrm{Al}_{2} \mathrm{O}_{3} \quad(99 \%)$ & .5 & $\begin{array}{r}7 \\
14 \\
21 \\
1\end{array}$ & $\begin{array}{l}0.34 \\
0.38 \\
0.56 \\
0.097\end{array}$ & $\begin{array}{l}4.9 \times 10^{-5} \\
2.7 \times 10^{-5} \\
2.7 \times 10^{-5} \\
9.7 \times 10^{-5}\end{array}$ & Localized Attack \\
\hline $\mathrm{TiO}_{2}$ & 15 & $\begin{array}{r}23 \\
7 \\
14 \\
1\end{array}$ & $\begin{array}{l}0.75 \\
0.46 \\
0.70 \\
32\end{array}$ & $\begin{array}{l}3.3 \times 10^{-5} \\
6.6 \times 10^{-5} \\
5.0 \times 10^{-5} \\
3.2 \times 10^{-2}\end{array}$ & Sample Cracked \\
\hline $\mathrm{CaTiO}_{3}$ & & $\begin{array}{r}23 \\
7 \\
14 \\
1\end{array}$ & $\begin{array}{l}1.93 \\
0.36 \\
1.3 \\
0.4\end{array}$ & $\begin{array}{l}8.4 \times 10^{-5} \\
5.1 \times 10^{-5} \\
9.3 \times 10^{-5} \\
4.0 \times 10^{-4}\end{array}$ & \\
\hline CaTiSiO 5 & & $\begin{array}{r}23 \\
7 \\
14 \\
1\end{array}$ & $\begin{array}{l}1.0 \\
0.97 \\
1.0 \\
0.45\end{array}$ & $\begin{array}{l}4.0 \times 10^{-5} \\
1.4 \times 10^{-4} \\
7.1 \times 10^{-5} \\
4.5 \times 10^{-4}\end{array}$ & \\
\hline $\mathrm{BaZrO}_{3}$ & & $\begin{array}{r}23 \\
7 \\
14 \\
1\end{array}$ & $\begin{array}{l}1.1 \\
0.32 \\
5.6 \\
1.9\end{array}$ & $\begin{array}{l}4.8 \times 10^{-5} \\
4.6 \times 10^{-5} \\
4.0 \times 10^{-4} \\
1.9 \times 10^{-3}\end{array}$ & $\begin{array}{l}\text { Alteration Reaction } \\
\text { Sample Cracked }\end{array}$ \\
\hline $\mathrm{CaZrO}_{3}$ & & $\begin{array}{r}23 \\
7 \\
14 \\
1\end{array}$ & $\begin{array}{l}0.8 \\
2.7 \\
3.4 \\
1.1\end{array}$ & $\begin{array}{l}3.5 \times 10^{-5} \\
3.9 \times 10^{-4} \\
2.4 \times 10^{-4} \\
1.1 \times 10^{-3}\end{array}$ & Alteration Reaction \\
\hline $\begin{array}{l}\text { Mullite } \\
\left(2 \mathrm{Al}_{2} \mathrm{O}_{3} \cdot \mathrm{SiO}_{2}\right)\end{array}$ & & $\begin{array}{r}7 \\
14 \\
21 \\
1\end{array}$ & $\begin{array}{l}2.3 \\
3.0 \\
3.7 \\
1.3\end{array}$ & $\begin{array}{l}3.3 \times 10^{-4} \\
2.1 \times 10^{-4} \\
1.8 \times 10^{-4} \\
1.3 \times 10^{-3}\end{array}$ & Alteration Reaction \\
\hline Basalt & $\lambda$ & $\begin{array}{r}23 \\
7 \\
14 \\
1\end{array}$ & $\begin{array}{l}4.3 \\
2.7 \\
4.3 \\
2.3\end{array}$ & $\begin{array}{l}1.9 \times 10^{-4} \\
3.9 \times 10^{-4} \\
3.1 \times 10^{-4} \\
2.3 \times 10^{-3}\end{array}$ & Alteration Reaction \\
\hline
\end{tabular}


TABLE 13. (contd)

\begin{tabular}{|c|c|c|c|c|}
\hline Material & $\begin{array}{c}\text { Exposure, } \\
\text { days }\end{array}$ & $\begin{array}{c}\text { Specimen } \\
\text { Weight Loss, } \\
\mathrm{mg} / \mathrm{cm}^{2}(\mathrm{~b})\end{array}$ & $\begin{array}{r}\text { Average Leach } \\
\text { Rate, (c) } \mathrm{g} / \mathrm{cm}^{2}-\mathrm{d}(\mathrm{b}) \\
\end{array}$ & Remarks \\
\hline $\begin{array}{l}\text { Vitreous Silica } \\
\left(\mathrm{SiO}_{2}\right)\end{array}$ & $\begin{array}{r}7 \\
14 \\
21 \\
1\end{array}$ & $\begin{array}{l}6.0 \\
7.2 \\
5.5 \\
2.0\end{array}$ & $\begin{array}{l}8.6 \times 10^{-4} \\
5.1 \times 10^{-4} \\
2.6 \times 10^{-4} \\
2.0 \times 10^{-3}\end{array}$ & \\
\hline Pyrocer am 9617 & $\begin{array}{r}7 \\
14 \\
21 \\
1\end{array}$ & $\begin{array}{l}6.0 \\
6.6 \\
8.5 \\
4.7\end{array}$ & $\begin{array}{l}8.6 \times 10^{-4} \\
4.7 \times 10^{-4} \\
4.0 \times 10^{-4} \\
4.7 \times 10^{-3}\end{array}$ & Alteration Reaction \\
\hline $\mathrm{ZrSiO}_{4}$ & $\begin{array}{r}23 \\
7 \\
14 \\
1\end{array}$ & $\begin{array}{c}17 \\
20 \\
13 \\
3.3\end{array}$ & $\begin{array}{l}7.4 \times 10^{-4} \\
2.9 \times 10^{-3} \\
9.3 \times 10^{-4} \\
3.3 \times 10^{-4}\end{array}$ & \\
\hline Marcor & $\begin{array}{r}7 \\
14 \\
21 \\
1\end{array}$ & $\begin{array}{l}20 \\
22 \\
27 \\
3.5\end{array}$ & $\begin{array}{l}2.9 \times 10^{-3} \\
1.6 \times 10^{-3} \\
1.3 \times 10^{-3} \\
3.5 \times 10^{-3}\end{array}$ & $\begin{array}{l}\text { Alteration Reaction } \\
\text { Specimen Cracked }\end{array}$ \\
\hline $\mathrm{BaTi0}_{3}$ & $\begin{array}{r}23 \\
7 \\
14 \\
1\end{array}$ & $\begin{array}{l}16 \\
35 \\
19\end{array}$ & $\begin{array}{r}7.0 \times 10^{-4} \\
5.0 \times 10^{-3} \\
1.4 \times 10^{-3} \\
\text { Shattered }\end{array}$ & \\
\hline
\end{tabular}

(a) $S / V \cong 0.4 \mathrm{~cm}^{-1}$ based on geometric surface area of the specimen.

(b) Based on geometric surface area of specimen.

(c) Average leach rate for exposure time shown.

of the other materials may exhibit a high leach resistance in one or two tests, but on an overall basis they are much inferior to the five materials mentioned.

The rankings in Table 15 are based only on the measured leach rates. They do not take into account the effects that localized attack or alteration reactions can have on the stability of candidate materials. These factors are considered in Section 4.3.

\subsection{REACTIONS WITH REPOSITORY MEDIA}

A limited number of experiments were carried out to determine if the ceramic materials would react with the repository media in a "dry" system. In the tests a sample of the candidate material was contacted with the repository medium (either synthetic WIPP salt or crushed basalt) in a sealed silica ampule at $375^{\circ} \mathrm{C}$. The tests lasted for 2200 and $4500 \mathrm{~h}$. At the conclusion of the test the silica ampule was opened and the sample was carefully separated from the bulk of the salt or basalt. The sample was then examined visually for evidence of possible reaction with the salt or basalt, after which it was weighed. In none of the tests was there any visual evidence of reaction between the test specimen and the salt or basalt. None of the spec imens exhibited any significant weight changes. 
TABLE 14. Leaching of Ceramic Materials in Brine at $250^{\circ} \mathrm{C}--$ Static Tests in Sealed Gold

\begin{tabular}{|c|c|c|c|c|}
\hline Material & $\begin{array}{c}\text { Exposure, } \\
\text { days }\end{array}$ & $\begin{array}{c}\text { Spec imen } \\
\text { Weight } \text { Loss, } \\
\mathrm{mg} / \mathrm{cm}^{2}(\mathrm{~b}) \\
\end{array}$ & $\begin{array}{r}\text { Aver age Leach } \\
\text { Rate, },(c)_{\mathrm{g} / \mathrm{cm}^{2}-d}(b) \\
\end{array}$ & Remarks \\
\hline Graphite & $\begin{array}{r}7 \\
14 \\
21 \\
1\end{array}$ & $\begin{array}{l}\text { wt. gain } \\
\text { wt. gain } \\
\text { wt. gain } \\
\text { wt. gain }\end{array}$ & $\begin{array}{l}-- \\
-- \\
--- \\
--\end{array}$ & \\
\hline $\mathrm{Al}_{2} \mathrm{O}_{3}(99.8 \%)$ & $\begin{array}{r}7 \\
14 \\
21 \\
1\end{array}$ & $\begin{array}{l}0.028 \\
0.094 \\
0.091 \\
0.018\end{array}$ & $\begin{array}{l}4.0 \times 10^{-6} \\
6.7 \times 10^{-6} \\
4.3 \times 10^{-6} \\
1.8 \times 10^{-5}\end{array}$ & \\
\hline $\mathrm{ZrO}_{2}$ & $\begin{array}{r}7 \\
14 \\
21 \\
1\end{array}$ & $\begin{array}{l}0.10 \\
1.3 \\
0.10 \\
0.11\end{array}$ & $\begin{array}{l}1.4 \times 10^{-5} \\
9.3 \times 10^{-5} \\
0.48 \times 10^{-5} \\
1.1 \times 10^{-4}\end{array}$ & \\
\hline $\mathrm{Al}_{2} \mathrm{O}_{3}(99 \%)$ & $\begin{array}{r}7 \\
14 \\
21 \\
1\end{array}$ & $\begin{array}{l}0.29 \\
0.39 \\
0.38 \\
0.096\end{array}$ & $\begin{array}{l}4.1 \times 10^{-5} \\
2.8 \times 10^{-5} \\
1.8 \times 10^{-5} \\
9.6 \times 10^{-5}\end{array}$ & Localized Attack \\
\hline $\mathrm{TiO}_{2}$ & $\begin{array}{r}23 \\
7 \\
14 \\
1\end{array}$ & $\begin{array}{l}0.79 \\
0.50 \\
0.61 \\
0.22\end{array}$ & $\begin{array}{l}3.4 \times 10^{-5} \\
7.1 \times 10^{-5} \\
4.4 \times 10^{-5} \\
2.2 \times 10^{-4}\end{array}$ & \\
\hline $\mathrm{CaTiO}_{3}$ & $\begin{array}{r}23 \\
7 \\
14 \\
1\end{array}$ & $\begin{array}{l}1.1 \\
0.58 \\
0.60 \\
0.1\end{array}$ & $\begin{array}{l}4.8 \times 10^{-5} \\
8.3 \times 10^{-5} \\
4.3 \times 10^{-5} \\
1.0 \times 10^{-4}\end{array}$ & \\
\hline $\begin{array}{l}\text { Mullite } \\
\left(2 \mathrm{Al}_{2} \mathrm{O}_{3} \cdot \mathrm{SiO}_{2}\right)\end{array}$ & $\begin{array}{r}7 \\
14 \\
21 \\
1\end{array}$ & $\begin{array}{l}1.5 \\
1.3 \\
0.9 \\
1.0\end{array}$ & $\begin{array}{l}2.4 \times 10^{-4} \\
9.3 \times 10^{-5} \\
4.3 \times 10^{-5} \\
1.0 \times 10^{-2}\end{array}$ & Alteration Reaction \\
\hline $\mathrm{BaZrO}_{3}$ & $\begin{array}{r}23 \\
7 \\
14 \\
1\end{array}$ & $\begin{array}{l}1.9 \\
0.94 \\
1.1 \\
13.1\end{array}$ & $\begin{array}{l}8.3 \times 10^{-5} \\
1.3 \times 10^{-4} \\
7.9 \times 10^{-5} \\
1.3 \times 10^{-2}\end{array}$ & $\begin{array}{l}\text { Alteration Reaction } \\
\text { Specimen Cracked }\end{array}$ \\
\hline $\begin{array}{l}\text { Vitreous Silica } \\
\left(\mathrm{SiO}_{2}\right)\end{array}$ & $\begin{array}{r}7 \\
14 \\
21 \\
1\end{array}$ & $\begin{array}{l}3.9 \\
5.1 \\
1.0\end{array}$ & $\begin{array}{c}5.6 \times 10^{-4} \\
\text { Ampule Leaked } \\
2.4 \times 10^{-4} \\
1.0 \times 10^{-3}\end{array}$ & \\
\hline Pyroceram 9617 & $\begin{array}{r}7 \\
14 \\
21 \\
1\end{array}$ & $\begin{array}{r}1.9 \\
6.3 \\
11 \\
1.6\end{array}$ & $\begin{array}{l}2.7 \times 10^{-4} \\
4.5 \times 10^{-4} \\
5.2 \times 10^{-4} \\
1.6 \times 10^{-3}\end{array}$ & Specimen Cracked \\
\hline $\mathrm{ZrSiO}_{4}$ & $\begin{array}{r}23 \\
7 \\
14 \\
1\end{array}$ & $\begin{array}{r}7.6 \\
10.7 \\
13.3 \\
2.4\end{array}$ & $\begin{array}{l}3.3 \times 10^{-4} \\
1.5 \times 10^{-3} \\
9.5 \times 10^{-4} \\
2.4 \times 10^{-3}\end{array}$ & \\
\hline
\end{tabular}


TABLE 14. (contd)

\begin{tabular}{|c|c|c|c|c|}
\hline Material & $\begin{array}{c}\text { Exposure, } \\
\text { days }\end{array}$ & $\begin{array}{c}\text { Specimen } \\
\text { Weight Loss, } \\
\mathrm{mg} / \mathrm{cm}^{2}(\mathrm{~b})\end{array}$ & $\begin{array}{c}\text { Average Leach } \\
\text { Rate, }(c) \mathrm{g} / \mathrm{cm}^{2}-\mathrm{d}(\mathrm{b})\end{array}$ & Remarks \\
\hline Marcor & $\begin{array}{r}7 \\
14 \\
21 \\
1\end{array}$ & $\begin{array}{l}12 \\
16 \\
39 \\
3.8\end{array}$ & $\begin{array}{l}1.7 \times 10^{-3} \\
1.1 \times 10^{-3} \\
1.9 \times 10^{-3} \\
3.8 \times 10^{-3}\end{array}$ & $\begin{array}{l}\text { Alteration Reaction } \\
\text { Specimen Cracked }\end{array}$ \\
\hline $\mathrm{BaTiO}_{3}$ & $\begin{array}{r}23 \\
7 \\
14 \\
1\end{array}$ & $\begin{array}{l}27 \\
14 \\
22 \\
53\end{array}$ & $\begin{array}{l}1.1 \times 10^{-3} \\
2.0 \times 10^{-3} \\
1.6 \times 10^{-3} \\
5.3 \times 10^{-2}\end{array}$ & Spec imen Cracked \\
\hline
\end{tabular}

(a) $S / V \cong 0.4 \mathrm{~cm}^{-1}$ based on geometric surface area of the specimen.

(b) Based on geometric surface area of specimen.

(c) Average leach rate for exposure time shown.

\subsection{EVALUATION OF CANDIDATE MATERIALS}

\subsubsection{Graphite}

The properties of graphite can vary significantly, depending on the grade of graphite being considered. The material used in this study was a low-permeability graphite (Toyo-Tanso IG-11) ${ }^{\circledR}$ developed for use in high-temperature, gas-cooled reactors.

Overall, the graphite exhibited the greatest resistance to attack by the leach solutions of any of the materials tested. Photomicrographs of the graphite specimens before and after testing provided no visual evidence of attack by the leach solutions in the dynamic tests or in the static tests at $150^{\circ} \mathrm{C}$ and lower. In the static tests at $250^{\circ} \mathrm{C}$, however, the micrographs do show some indication of corrosion (see Figure 3 ).

Analysis of leach solutions from the static tests showed that small quantities of aluminum, sodium, and silicon were leached from the graphite. The amount of impurities leached from the graphite samples, however, accounted for on 1 y $10 \%$ to $20 \%$ of the weight loss exhibited by the graphite in the various leach tests. The bulk of the weight loss in each case was probably due to the reaction of the graphite with dissolved oxygen in the leach solution. This could explain why the graphite exhibited slightly greater leach rates in the static test.s at $250^{\circ} \mathrm{C}$ than in the tests with flowing demineralized water at $250^{\circ} \mathrm{C}$. In the static leach tests the gold ampules were only partially filled with leach solution. The remaining space was filled with air, and during testing the oxygen in the air could dissolve in the leach solution and react with the graphite. Calculations show that the air contained in the sealed gold ampules could supply sufficient oxygen to account for up to $80 \%$ of the weight loss exhibited by the graphite samples in the static tests at $250^{\circ} \mathrm{C}$, and all of the weight loss in the $150^{\circ} \mathrm{C}$ static tests. Gray (1980) studied the reaction of graphite powder with water at $200^{\circ}$ to $300^{\circ} \mathrm{C}$ and reported that $h$ is results indicated the carbon reacted with dissolved oxygen rather than with water.

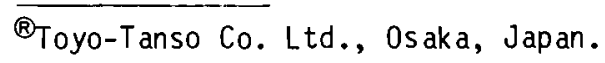


TABLE 15. Relative Resistance of Ceramic Materials to Leaching Under Various Test Conditions (in Order of Decreasing Resistance)

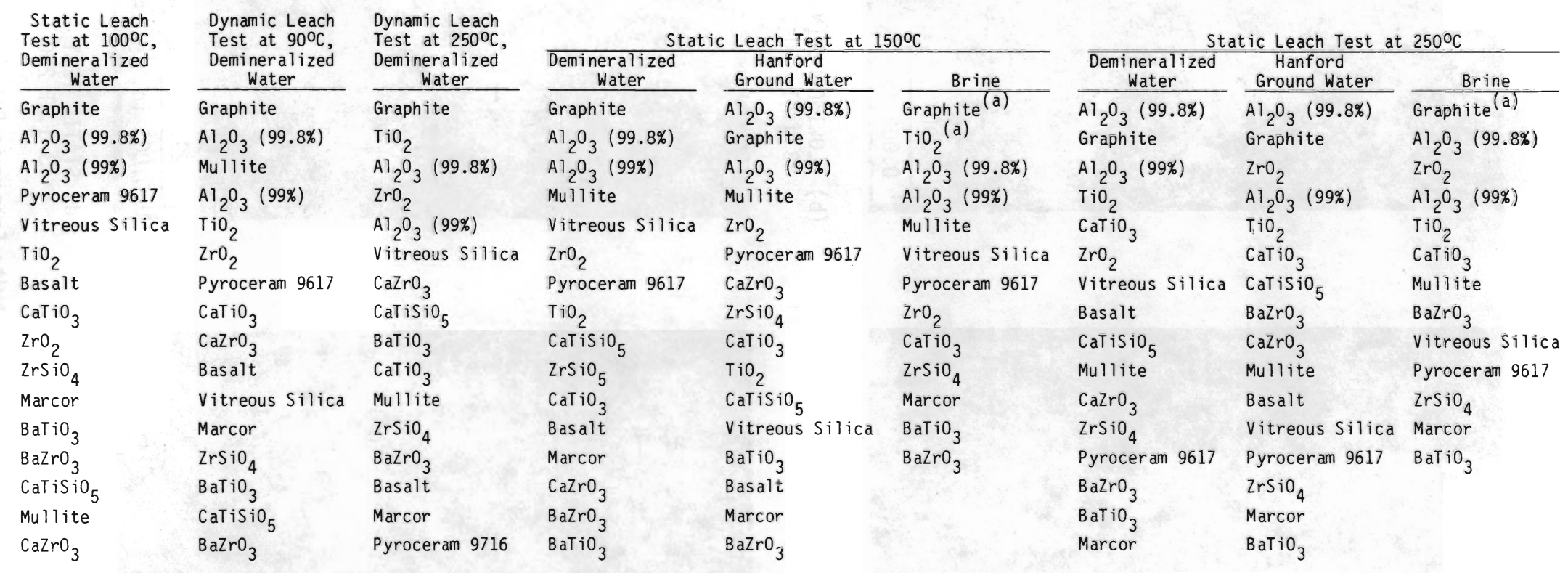

(a) Test specimens exhibited weight gains due to incomplete removal of brine prior to drying. 


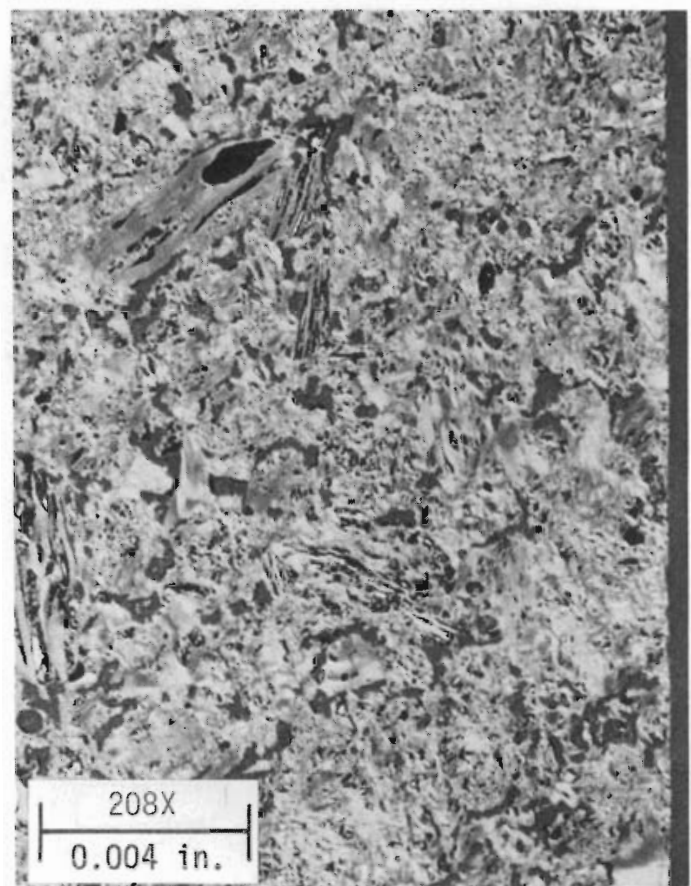

(a) AS-RECEIVED

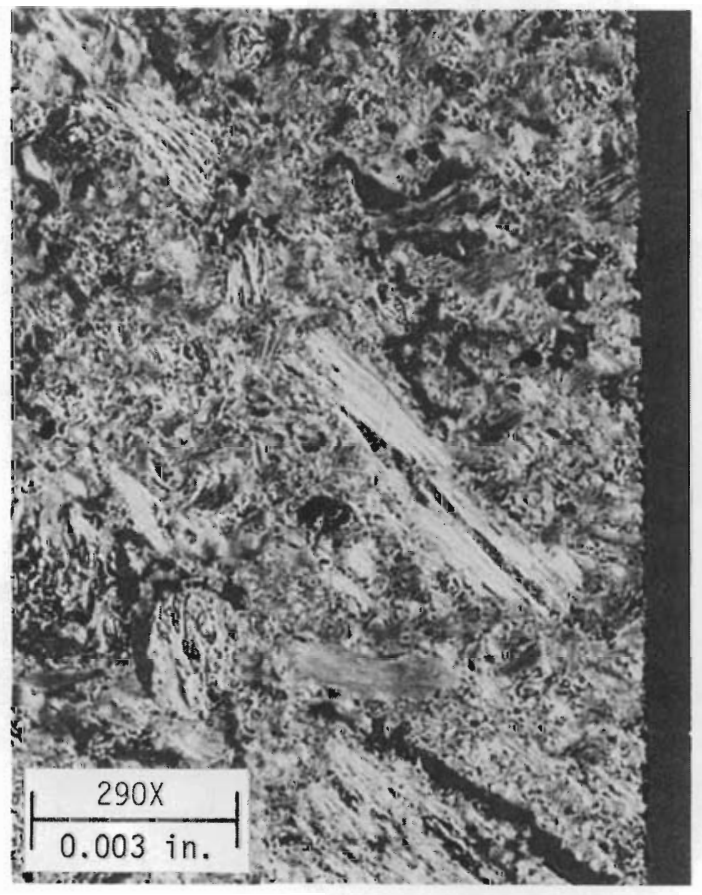

(c) STATIC TEST - DEMINERALIZED WATER, 43 d AT $250^{\circ} \mathrm{C}$

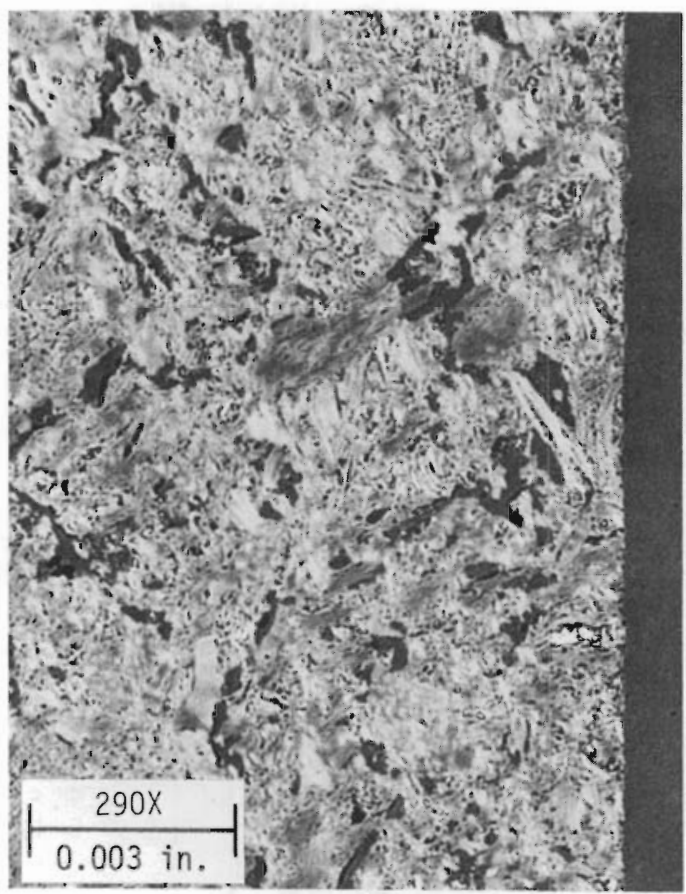

(b) FLOWING DEMINERALIZED WATER, 43 d AT $250^{\circ} \mathrm{C}$

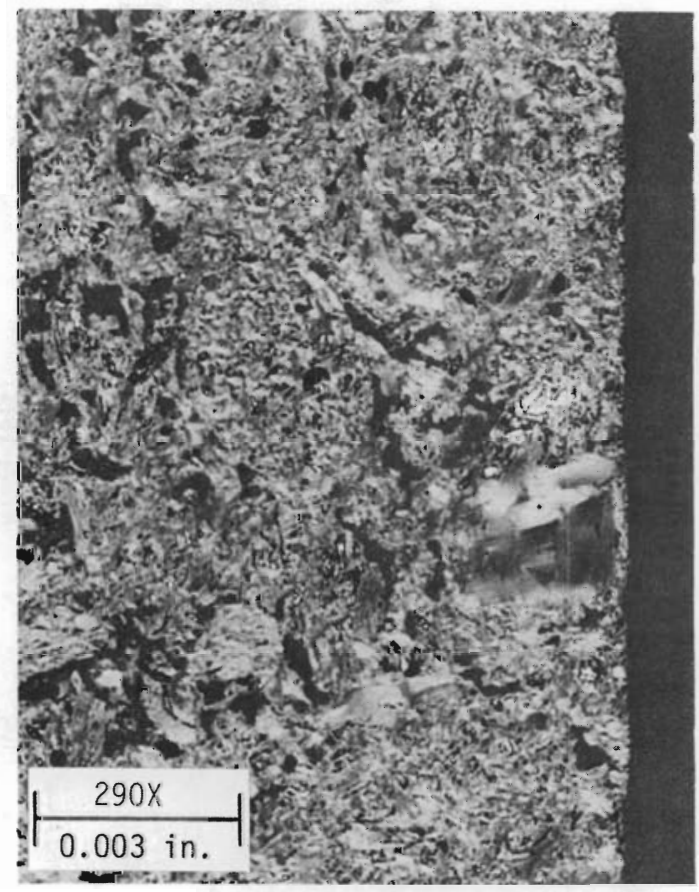

(d) STATIC TEST - BRINE SOLUTION, 43 d AT $250^{\circ} \mathrm{C}$

FIGURE 3. Photomicrographs of Graphite Specimens Before and After Testing 
The graphite used in this study was permeable, and the test samples had total surface areas, as determined by the BET method, that were 2000 to 3000 times greater than their geometric surface areas. If the leach rates are calculated on the basis of total surface areas, the values for graphite under the various test conditions would range from $10^{-11}$ to $10^{-8} \mathrm{~g} /$ $\mathrm{cm}^{2}$-d. Gray (1980) reported reaction rates ranging from $5 \times 10^{-11}$ to $5 \times 10^{-8} \mathrm{~g} / \mathrm{cm}^{2}-\mathrm{d}$ over the same temperature range.

Graphite possesses a number of properties that favor its selection as a barrier material over the other material tested. Graphite is readily available and relatively inexpensive. Some techniques are available for fabricating large shapes, and graphite is easily machined, which facilitates the use of special overpack canister closures. The permeability of the graphite can probably be reduced to an acceptable level using various impregnation techniques. Overall, the mechanical and physical properties of graphite are superior to those of the ceramic material tested. It has good thermal conductivity and thermal-shock resistance for a nonmetallic material--two properties that are critical to the development of barrier elements. Its mechanical properties should be adequate to permit its use as overpack or a hole sleeve.

The one major question that must be answered regarding the use of graphite as a barrier material is what effect radiation would have on the corrosion of the graphite by the liquids encountered in the respository. Detailed studies of graphite leaching in the presence of a radiation field are required to resolve this question. Some development work on the fabrication of large shapes of the size required for a barrier system would also be necessary.

\subsection{2 $\quad \underline{A l}_{2} \underline{0}_{3}$}

Overall, the leach resistance of $\mathrm{Al}_{2} \mathrm{O}_{3}$ was lower than that of graphite but superior to the other ceramic materials tested. Two grades of alumina were evaluated, the principal difference between the two being the impurity content. One grade contained approximately 0.2 wt\% impurities, while the second contained about 1 wt\% impurities. In every leach test the $\mathrm{Al}_{2} \mathrm{O}_{3} \mathrm{having}$ the lowest impurity content exhibited the lowest leach rate. Even at $250^{\circ} \mathrm{C}$ the aluminum concentration in the leach solutions was quite low and the dissolved alumina never accounted for more than $50 \%$ of the weight loss suffered by a test specimen. Analysis of the leach solutions showed that a number of cation impurities were leached from both grades of alumina with silicon, sodium, and calcium being the principal species removed. As would be expected, leaching of impurities from the $99 \% \mathrm{Al}_{2} \mathrm{O}_{3}$ was much greater than from the $99.8 \%$ material. Ceramographic examination of the test specimens after leaching showed extensive localized attack that apparently resulted from the leaching of impurities from the grain boundaries of the alumina.

Figures 4 and 5 show typical micrographs of $\mathrm{Al}_{2} \mathrm{O}_{3}$ before and after testing. It is obvious from the micrographs that, for a given leach test, grain boundary attack was substantially greater with the $99 \% \mathrm{Al}_{2} \mathrm{O}_{3}$ than with the $99.8 \% \mathrm{Al}_{2} \mathrm{O}_{3}$.

At $250^{\circ} \mathrm{C}$ both grades of alumina exhibit much higher leach rates in the flowing demineralized water than in the static tests. In the static tests the aluminum concentration in the leach solution appears to reach saturation in a relatively short period of time. As the aluminum concentration approaches saturation, the leach rate decreases accordingly, although even 


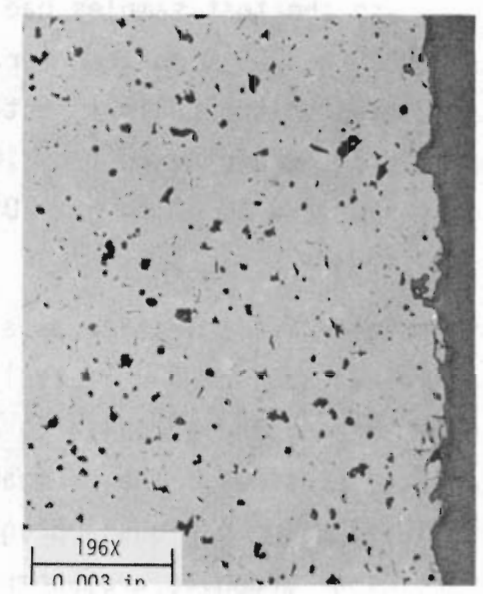

(a) AS-RECEIVED

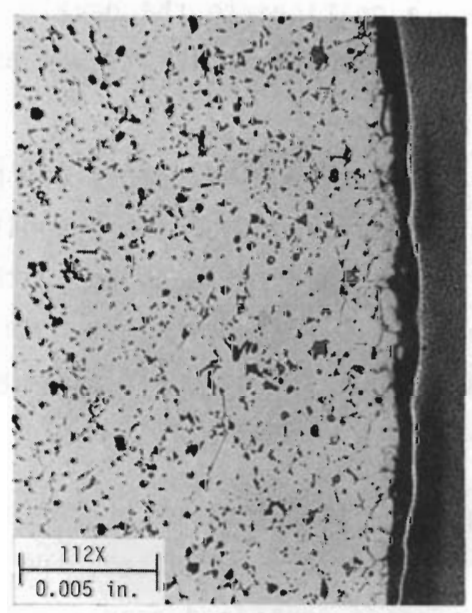

(c) STATIC TEST - DEMINERALIZED WATER,
$43 \mathrm{~d}$ AT $250^{\circ} \mathrm{C}$
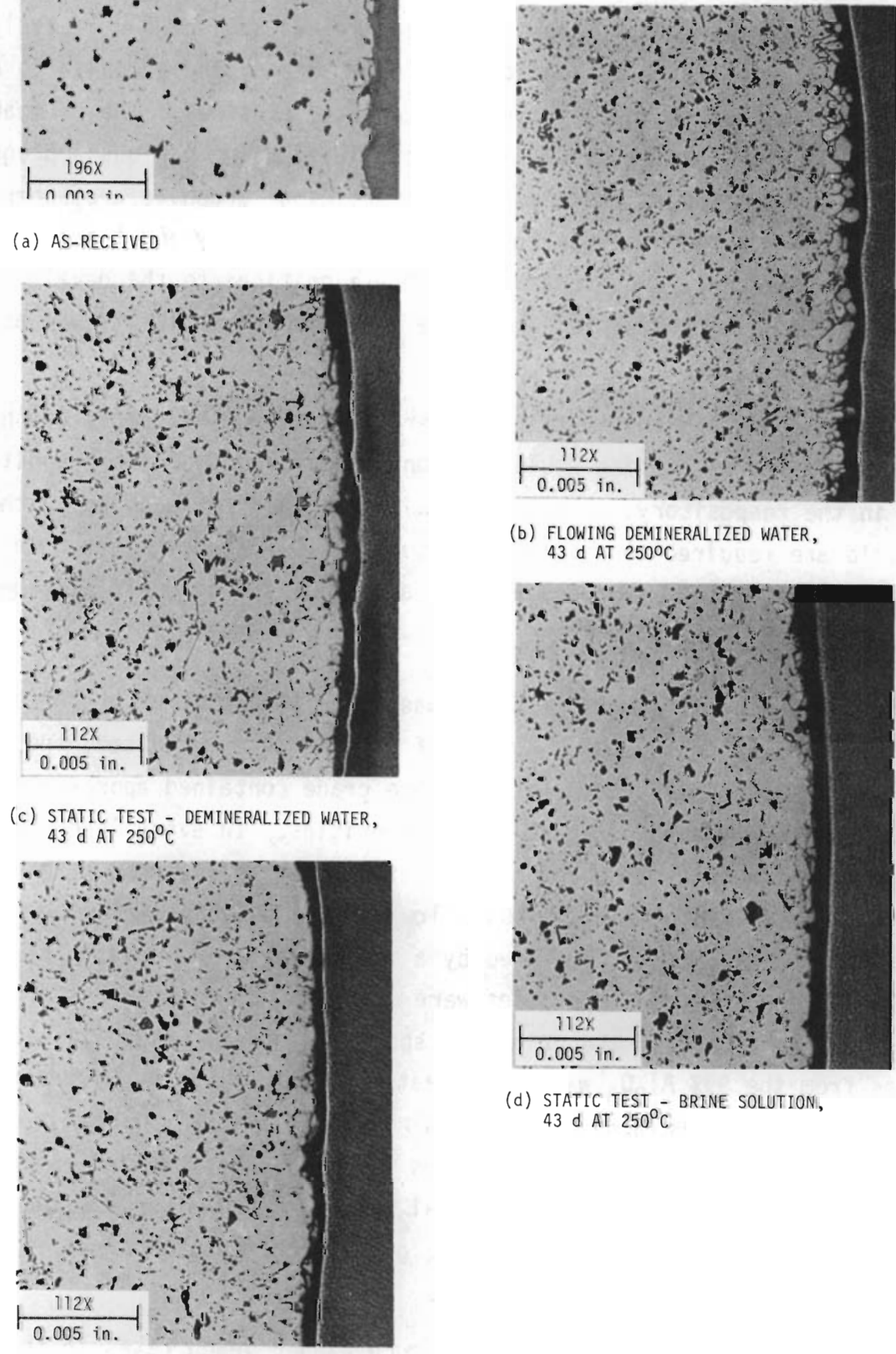

(b) FLOWING DEMINERALIZED WATER,

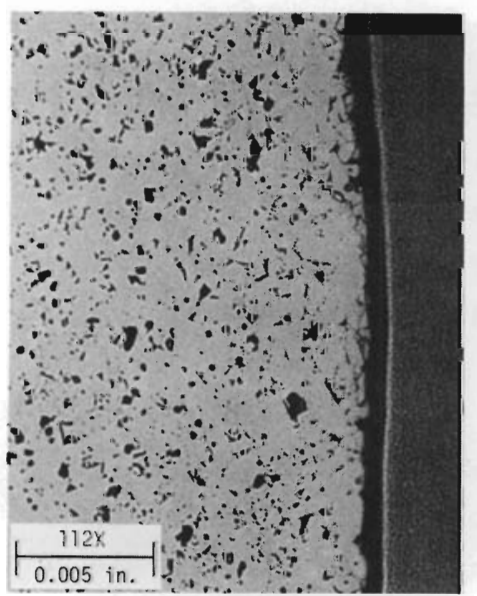

(d) STATIC TEST - BRINE SOLUTION, $43 \mathrm{~d}$ AT $250^{\circ} \mathrm{C}$

(e) STATIC TEST - GROUNDWATER,
$43 \mathrm{~d}$ AT $250^{\circ} \mathrm{C}$

FIGURE 4. $\mathrm{Al}_{2} \mathrm{O}_{3}(99 \%)$ Specimens Before and After Leaching 


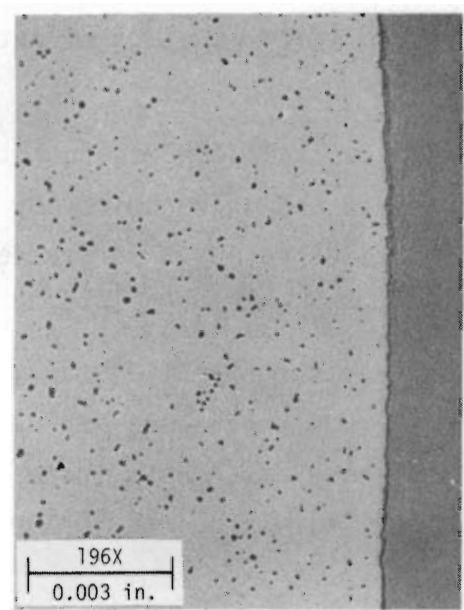

(a) AS-RECEIVED

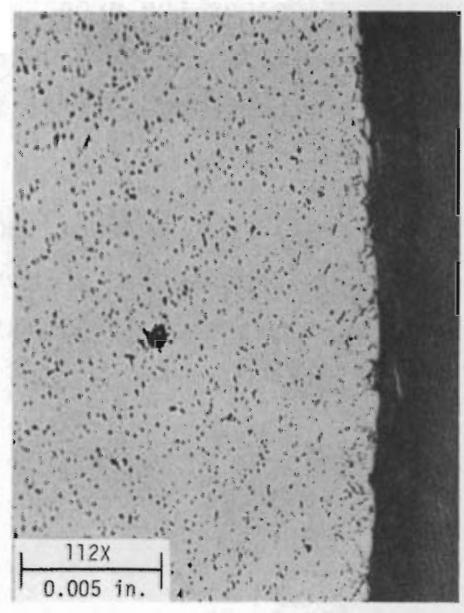

(c) STATIC TEST - DEMINERALIZED WATER,
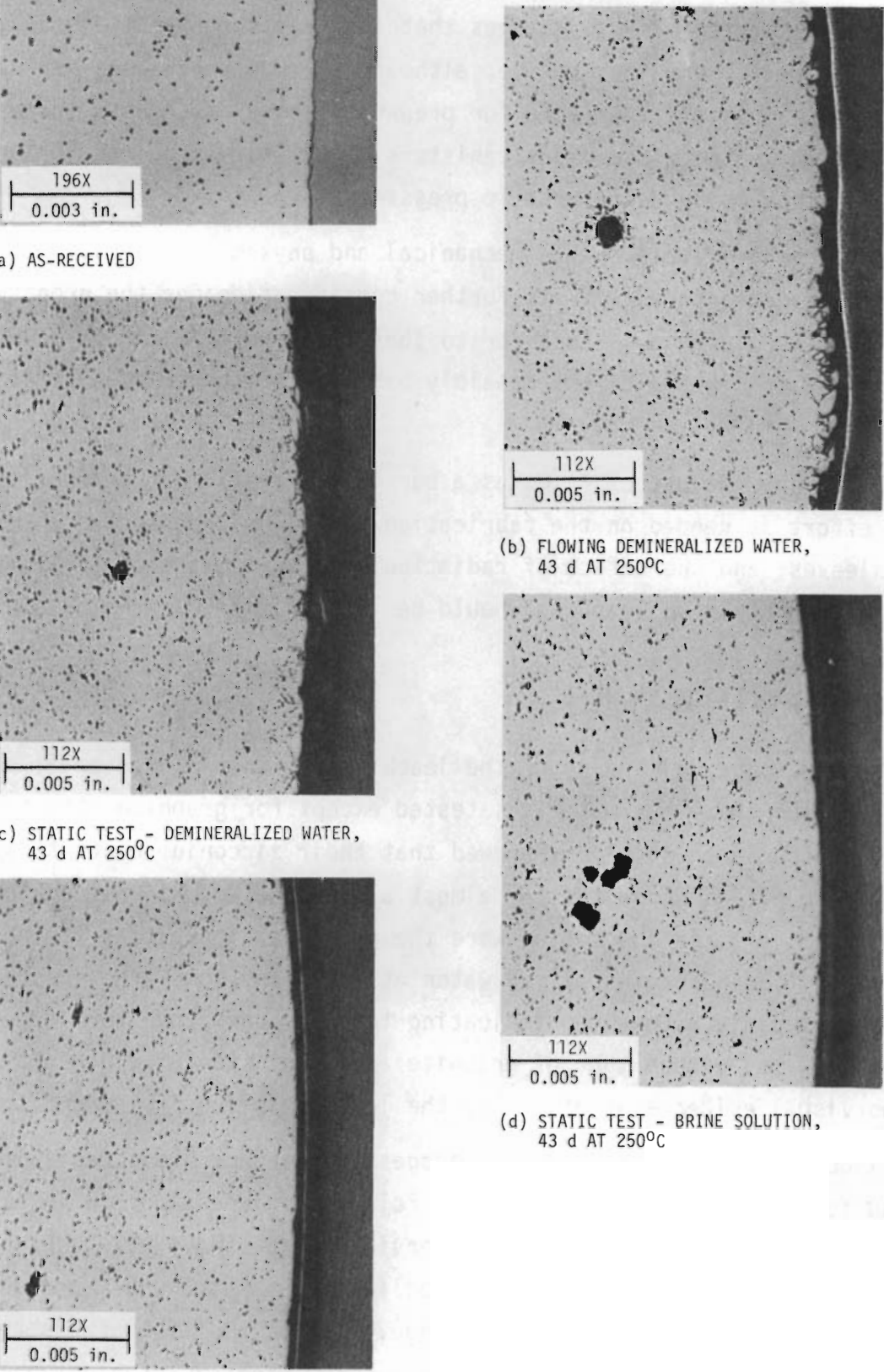

(b) FLOWING DEMINERALIZED WATER, 43 d AT $250^{\circ} \mathrm{C}$

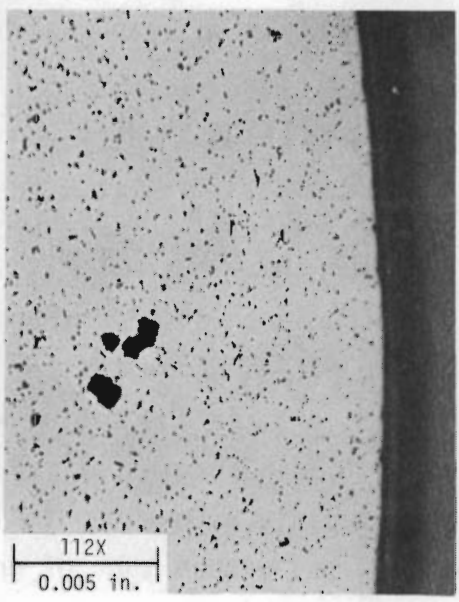
(d) STATIC TEST - BRINE SOLUTION,
$43 \mathrm{~d}$ AT $250^{\circ} \mathrm{C}$

(e) STATIC TEST - GROUNDWATER,

43 d AT $250^{\circ} \mathrm{C}$

FIGURE 5. $\mathrm{Al}_{2} \mathrm{O}_{3}(99.8 \%)$ Specimens Before and After Leaching 
at aluminum saturation some attack occurs due to the leaching of impurities from the grain boundaries. In the dynamic test at $250^{\circ} \mathrm{C}$ the flow rate of the demineralized water was sufficiently great that the aluminum concentration in the leach solution leaving the autoclave was very low--far below the apparent solubility limit.

In the static leach tests at $150^{\circ}$ and $250^{\circ} \mathrm{C}$ the apparent saturation concentrations for aluminum were highest in the demineralized water and lowest in the brine solution, with the ground water exhibiting intermediate levels. Reasons for the differences in apparent aluminum saturation concentrations for the different leach solutions were not determined.

Aluminum oxide possesses certain advantages that increase the potential for its use as a barrier element. It is relatively inexpensive, although the cost increases rapidly with increasing purity. Techniques are available for preparing dense impervious shapes of $\mathrm{Al}_{2} \mathrm{O}_{3}$, and work in Sweden has shown that impervious canisters of the size required for an engineeredbarrier system can be prepared by hot isostatic pressing.

Considerable data are available on the mechanical and physical properties of $\mathrm{Al}_{2} \mathrm{O}_{3}$, and its properties appear adequate to permit its further consideration for the proposed application. Its thermal-shock resistance is superior to that of any of the other ceramic materials tested, except for the vitreous silica and possibly basalt; its thermal conductivity is also relatively high.

Two problems regarding the use of $\mathrm{Al}_{2} \mathrm{O}_{3}$ as a barrier material must be resolved. Additional development effort is needed on the fabrication of full-sized barrier elements, such as overpacks or hole sleeves, and the effect of radiation on the leaching of $\mathrm{Al}_{2} \mathrm{O}_{3}$ must be determined. The effect of radiation on leaching should be less critical in the case of alumina than in the case of graphite.

\section{$4.3 .3 \quad \underline{\mathrm{Zr}}_{2}$}

Zirconium oxide did not excel in any of the leach tests, but on an overall basis it was clearly superior to all of the other materials tested except for graphite, the two aluminas, and $\mathrm{TiO}_{2}$. Analysis of the leach solutions showed that their zirconium contents were very low and that leaching of impurities accounted for almost all of the weight loss exhibited by the test specimens. Calcium, silicon and sodium were the principal impurities leached from the $\mathrm{ZrO}_{2}$. In the test with flowing demineralized water at $250^{\circ} \mathrm{C}$ and some other tests, the leach rate of $\mathrm{ZrO}_{2}$ decreased rapidly with time, indicating that for very long-term exposures the leach resistance of $\mathrm{ZrO}_{2}$ may exceed that of graphite and $\mathrm{TiO}_{2}$. Photomicrographs of the test specimens showed no visual evidence of attack by the leach solutions (Figure 6 ).

Zirconium dioxide possesses certain disadvantages that reduce its potential as a barrier material. Compared to graphite and alumina, it is relatively expensive and less readily available. Techniques for fabricating large, dense impervious shapes have not been developed. Zirconium oxide undergoes a transformation from a monoclinic to a tetragonal form when heated; the transition is accompanied by about a $9 \%$ volume change. By the addition of a stabilizer, such as $\mathrm{CaO}, \mathrm{MgO}$, or $\mathrm{Y}_{2} \mathrm{O}_{3}$, it is possible to produce a cubic $\mathrm{ZrO}_{2}$ that is stable above and below 


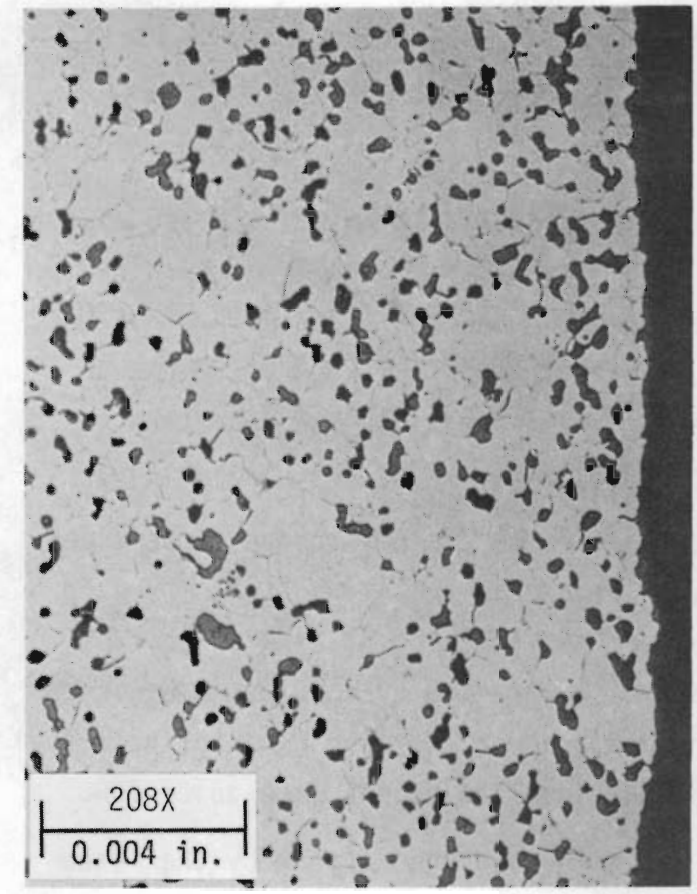

(a) AS-RECEIVED

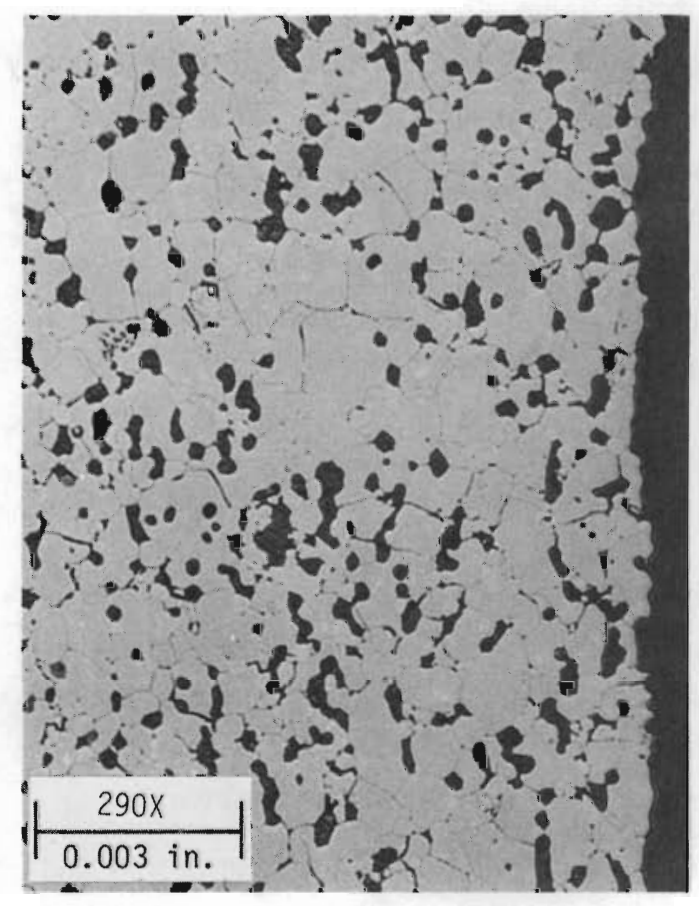

(c) STATIC TEST - GROUNDWATER,
$43 \mathrm{~d}$ AT $250^{\circ} \mathrm{C}$

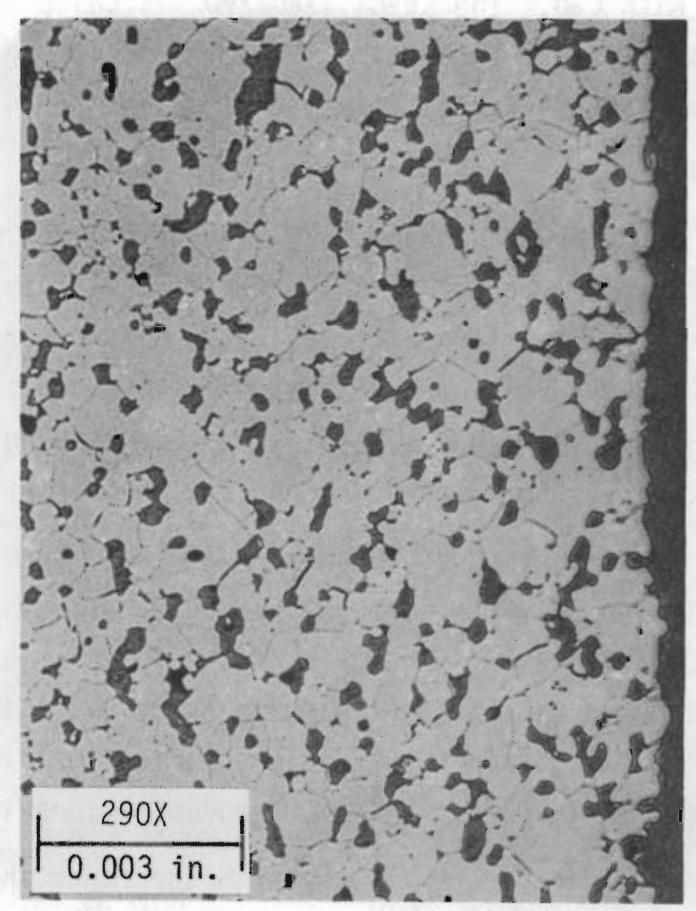

(b) FLOWING DEMINERALIZED WATER, 43 d AT $250^{\circ} \mathrm{C}$

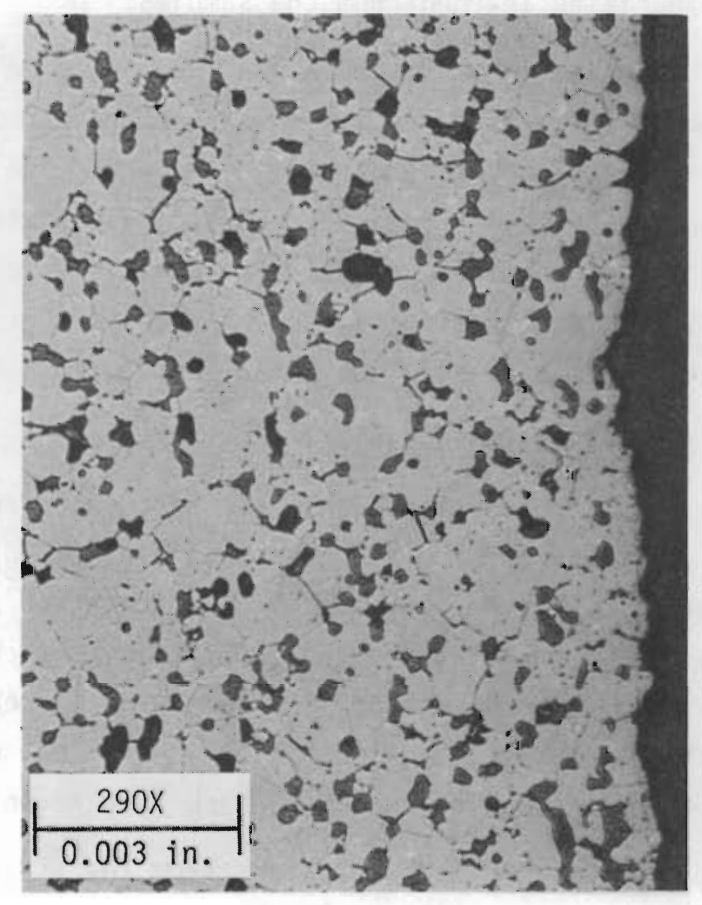

(d) STATIC TEST - BRINE SOLUTION, $43 \mathrm{~d} \mathrm{AT} 250^{\circ} \mathrm{C}$

FIGURE 6. $\quad \mathrm{ZrO}_{2}$ Specimens Before and After Leaching 
the monoclinic-tetragonal transformation iemperature. The $\mathrm{ZrO}_{2}$ used in this study was stabilized with $\mathrm{CaO}$. The stabilized $\mathrm{ZrO}_{2}$ is quite sensitive to thermal shock. The thermal conductivity of $\mathrm{ZrO}_{2}$ is relatively low and its other pertinent physical and mechanical properties appear to be inferior to those of graphite and alumina.

\subsection{4 $\mathrm{TiO}_{2}$}

On an overall basis, $\mathrm{TiO}_{2}$ was less leach resistant than graphite, alumina, and $\mathrm{ZrO}_{2}$ but was clearly superior to the other materials tested. In the test with flowing demineralized water at $250^{\circ} \mathrm{C}, \mathrm{TiO}_{2}$ exhibited a much lower leach rate than $\mathrm{ZrO}_{2}$ and the two aluminas but had to be down-graded because the specimen exhibited several radial cracks (Figure 7). Some of the other $\mathrm{TiO}_{2}$ samples also exhibited cracking. There is some indication, however, that the cracking was the result of fabrication problems rather than attack by the leach solutions.

$\mathrm{TiO}_{2}$ appeared to be unusual in two respects: the leach rates did not appear to be especially sensitive to temperature, and the leach rates were higher in the static tests than in the dynamic tests. Analysis of the leach solutions showed that leaching of impurities from the $\mathrm{TiO}_{2}$ accounted for the major fraction of the weight loss exhibited by the specimens in the various tests. Silicon, sodium, barium, calcium, magnesium, copper and iron were the principal impurities leached. Titanium concentrations in the various leach solutions were quite low.

Photomicrographs of the test specimens showed no visual evidence of attack by the leach solutions, except for the cracking observed with some samples (Figure 7). The localized attack apparent with $\mathrm{Al}_{2} \mathrm{O}_{3}$ was not obvious with $\mathrm{TiO}_{2}$, which is surprising, considering the quantity of impurities leached from the samples.

As a barrier material, $\mathrm{TiO}_{2}$ appears to be inferior to graphite and alumina in almost every respect; it is less readily available and more expensive. Techniques for fabricating large impervious shapes are not well developed. The physical properties of $\mathrm{TiO}_{2}$, such as thermal conductivity and thermal-shock sensitivity, are inferior to those of graphite and alumina. Only a limited amount of data are available on the mechanical properties of $\mathrm{TiO}_{2}$, but the data available indicate $\mathrm{TiO}_{2}$ is inferior to both graphite and $\mathrm{Al}_{2} \mathrm{O}_{3}$. The one advantage of $\mathrm{TiO}_{2}$ was the low leach rate exhibited in flowing demineralized water at $250^{\circ} \mathrm{C}$.

\subsubsection{Mullite $\left(2 \mathrm{Al}_{2}{ }_{2}{ }_{3} \cdot \mathrm{SiO}_{2}\right)$}

Evaluation of the leach rate data shows that, on an overall basis, mullite is much less resistant to leaching than are graphite, $\mathrm{Al}_{2} \mathrm{O}_{3}, \mathrm{ZrO}_{2}$, or $\mathrm{TiO}_{2}$. In addition, ceramographic examination of the test specimens showed that at $250^{\circ} \mathrm{C}$ mullite undergoes alteration reactions with the leach solutions that affect it to much greater depths than is apparent from the leach rate data. Micrographs of the specimens tested at $250^{\circ} \mathrm{C}$ are shown in Figure 8 and the extent of the alteration reactions is obvious. In the test with flowing demineralized water the entire area shown in the micrograph (Figure $8 \mathrm{~b}$ ) has undergone the alteration reaction.

Analysis of leach solutions from the static tests at $250^{\circ} \mathrm{C}$ showed that the amount of material dissolved from a given sample was 2 to 3 times greater than the sample's measured weight loss. This indicates that some form of hydration reaction probably occurred at the sample sur- 
(a) AS-FABRICATED
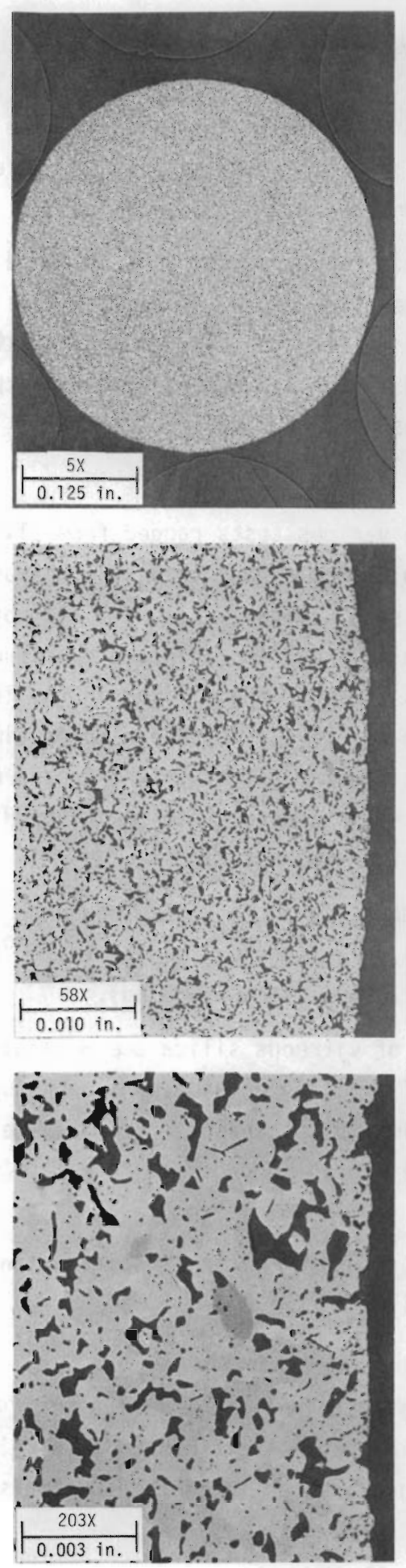

(b) FLOWING DEMINERALIZED WATER, 43 d AT $2500 \mathrm{C}$
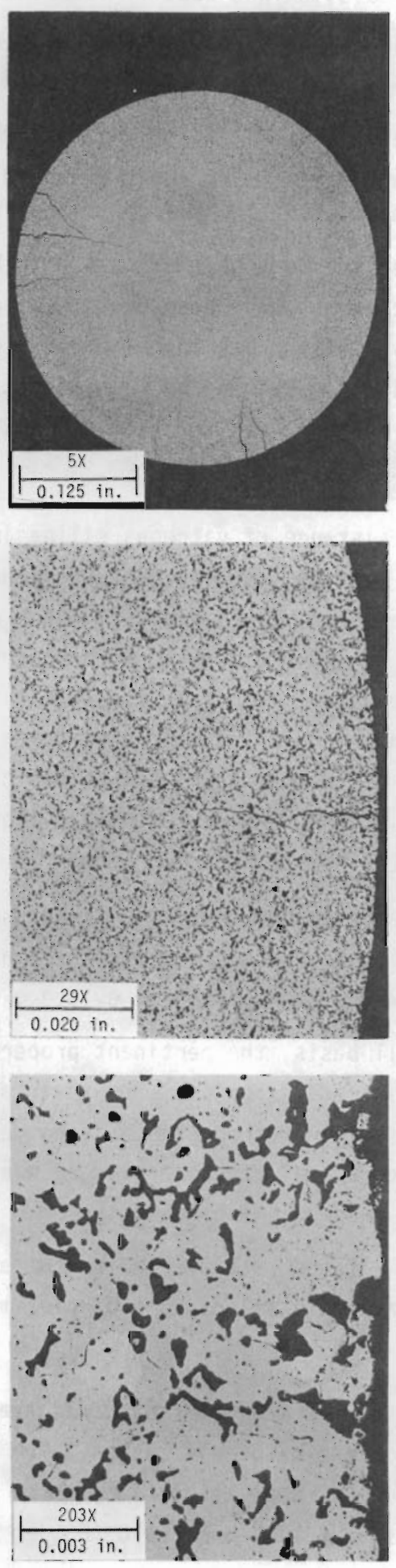

FIGURE 7. $\mathrm{TiO}_{2}$ Samples Before and After Leaching 
face. The analytical data showed that silicon was the principal cation leached from the mullite, together with minor amounts of aluminum, potassium, calcium, and sodium. The aluminum concentration in solution was usually less than $5 \%$ of the silicon concentration.

Ceramographic examination of mullite samples tested at $150^{\circ} \mathrm{C}$ provided no visual evidence that alteration reactions occurred with any of the leach solutions (see Figure $8 f$ ). Analysis of the leach solutions from the static tests at $150^{\circ} \mathrm{C}$ showed that silicon was the major species leached from the mullite. The aluminum concentrations in the leach solutions were low, amounting to no more than $10 \%$ of the silicon concentrations.

Mullite possesses many of alumina's attributes. It is readily available and relatively inexpensive. Techniques have been developed for preparing dense, impervious mullite shapes. The pertinent physical and mechanical properties of mullite, however, are generally inferior to those of alumina. Based on the results obtained in the various leach tests, there is little to recommend the further consideration of mullite as a barrier material.

\subsubsection{Vitreous Silica $\left(\mathrm{SiO}_{2}\right)$}

The leach resistance of vitreous silica in the various tests ranged from slightly above average to below average. Analyses of the leach sclutions showed that silicon was the principal species leached from the silica, together with small amounts of sodium and potassium. In the static tests at $150^{\circ} \mathrm{C}$ the silicon concentration in the leach solution continued to increase with increasing exposure time. In the static tests at $250^{\circ} \mathrm{C}$ the silicon in the leach solutions appeared to reach an equilibrium concentration in a relatively short period of time ( $\leq 7$ days). At both $150^{\circ}$ and $250^{\circ} \mathrm{C}$ the leach rate of silica was higher in the Hanford ground water than in the other two solutions. At $250^{\circ} \mathrm{C}$ the equilibrium silicon concentration in the Hanford ground water was about $220 \mathrm{ppm}$ and was much lower in the other two solutions.

Ceramographic examination of the silica specimens tested at $250^{\circ} \mathrm{C}$ showed no indication of localized attack or alteration reactions (Figure 9). The specimen tested at $150^{\circ} \mathrm{C}$ with $\mathrm{Hanford}$ ground water, however, did show some evidence of cracking (see Figure 8d).

On an overall basis, the pertinent properties of vitreous silica are generally inferior to those of alumina and graphite. One advantage of silica over alumina is its superior resistance to thermal shock. Methods for casting dense impervious shapes of silica are well developed, although additional development work would be needed in order to fabricate silica canisters of the size needed in a barrier system.

Based on all of the data available, there appears to be little justification in giving further consideration to silica as a barrier material.

\subsection{7 $\underline{\mathrm{CaTiO}}_{3}$}

The results of the leach tests showed that the relative leach resistance of $\mathrm{CaTiO}_{3}$ is near the medium of the materials tested. The sample exposed to flowing demineralized water at $250^{\circ} \mathrm{C}$ suffered some surface alteration, which was not apparent with the specimens subjected to the static tests at $150^{\circ}$ and $250^{\circ} \mathrm{C}$ (Figure 10). 


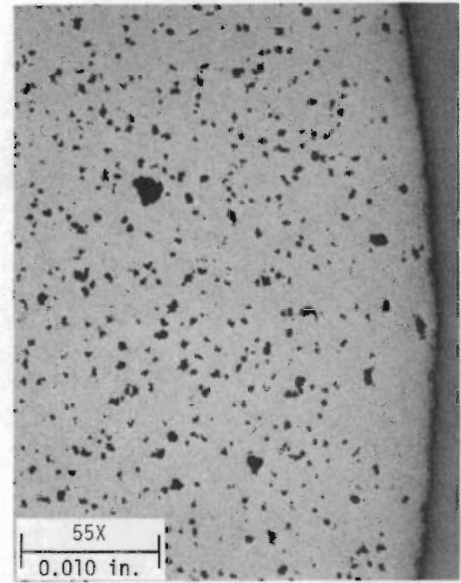

(a) AS-RECEIVED

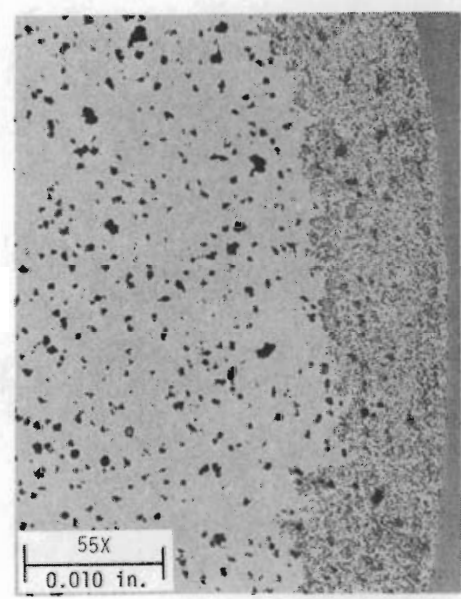

(c) STATIC TEST - DEMINERALIZED WATER,

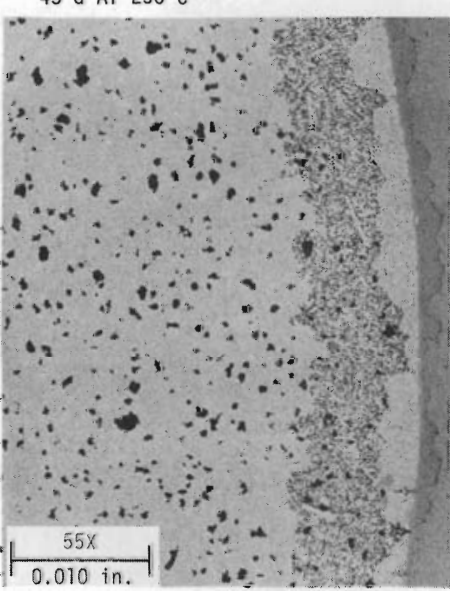

(e) STATIC TEST - BRINE SOLUTION,

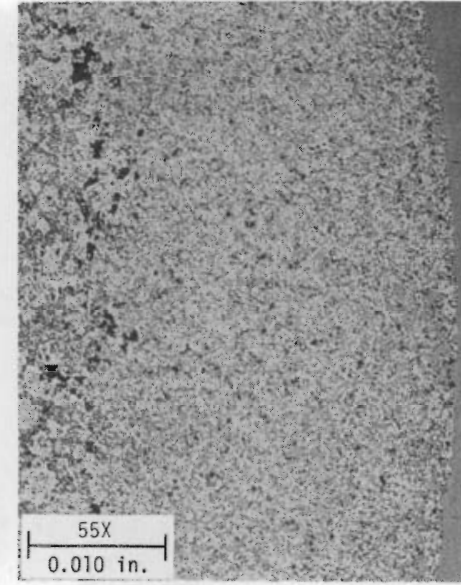

(b) FLOWING DEMINERALIZED WATER,

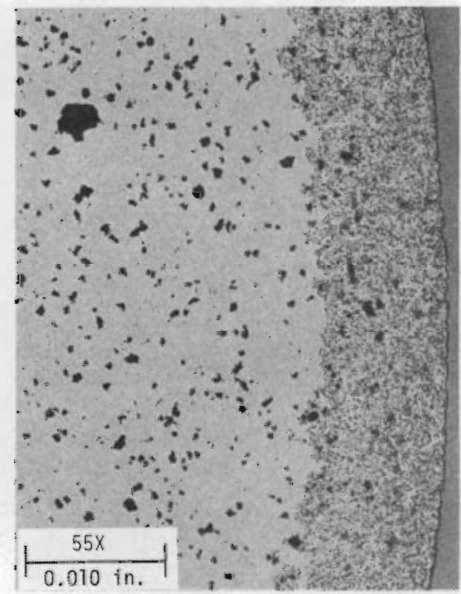

(d) STATIC TEST - GROUNDWATER,

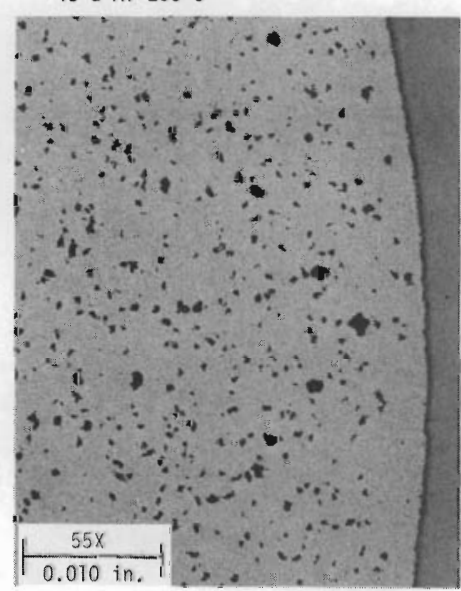

(f) STATIC TEST - DEMINERALIZED WATER, $43 \mathrm{~d}$ AT $150^{\circ} \mathrm{C}$

FIGURE 8. Micrographs of Mullite Test Specimens 


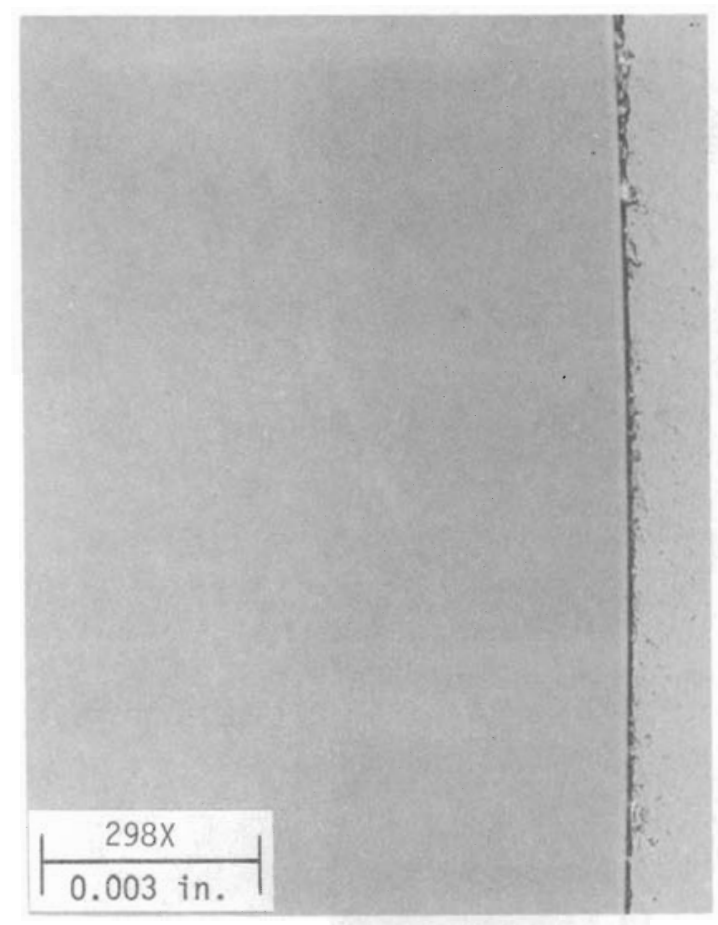

(a) AS-RECEIVED

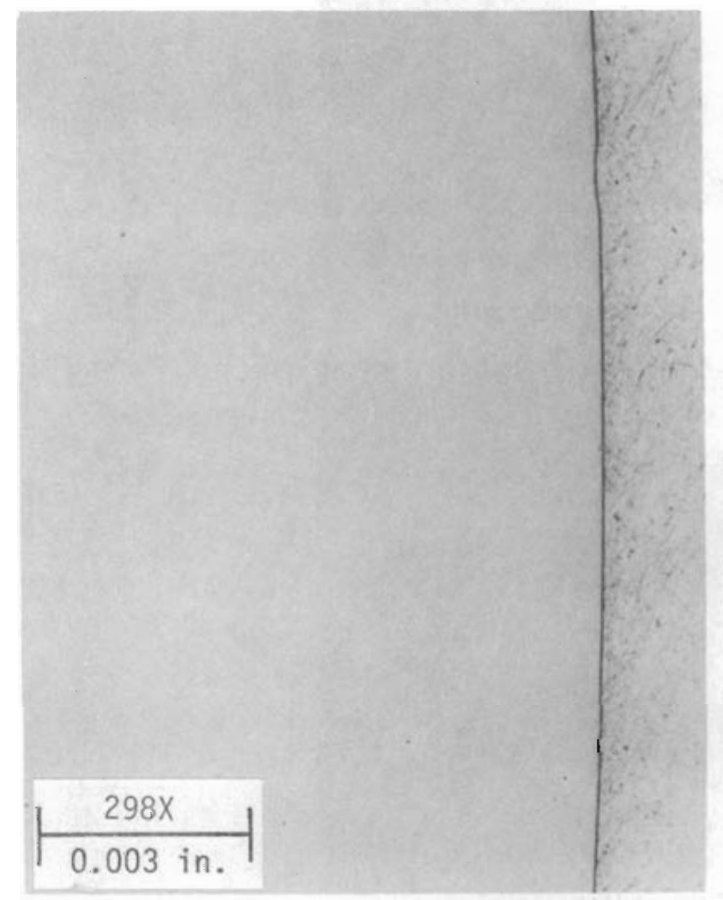

(c) STATIC TEST - HANFORD GROUNDWATER,

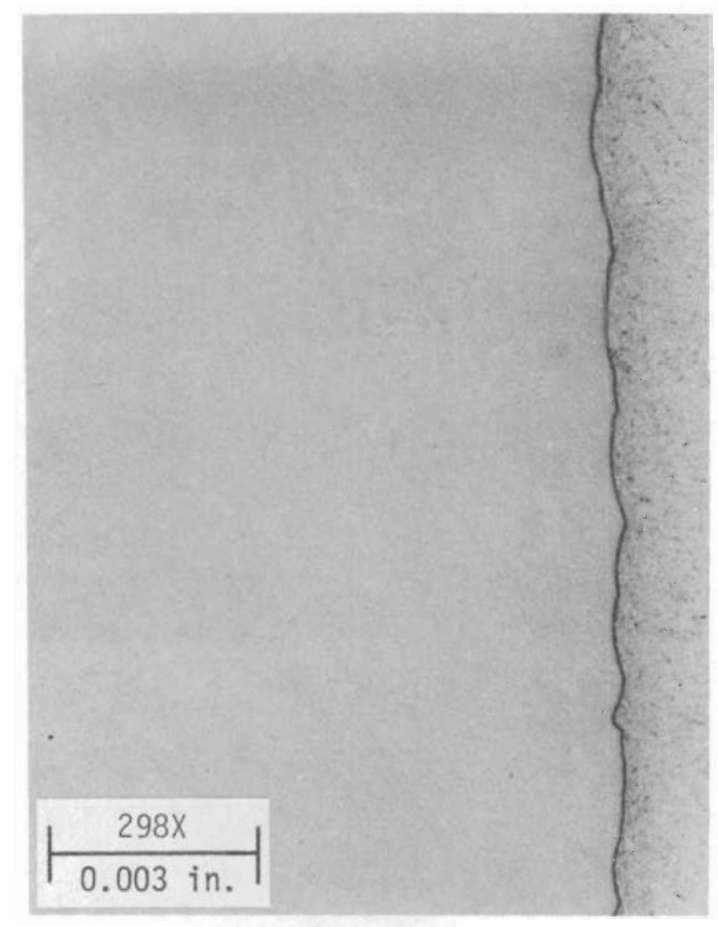

(b) FLOWING DEMINERALIZED WATER, 43 d AT $250^{\circ} \mathrm{C}$

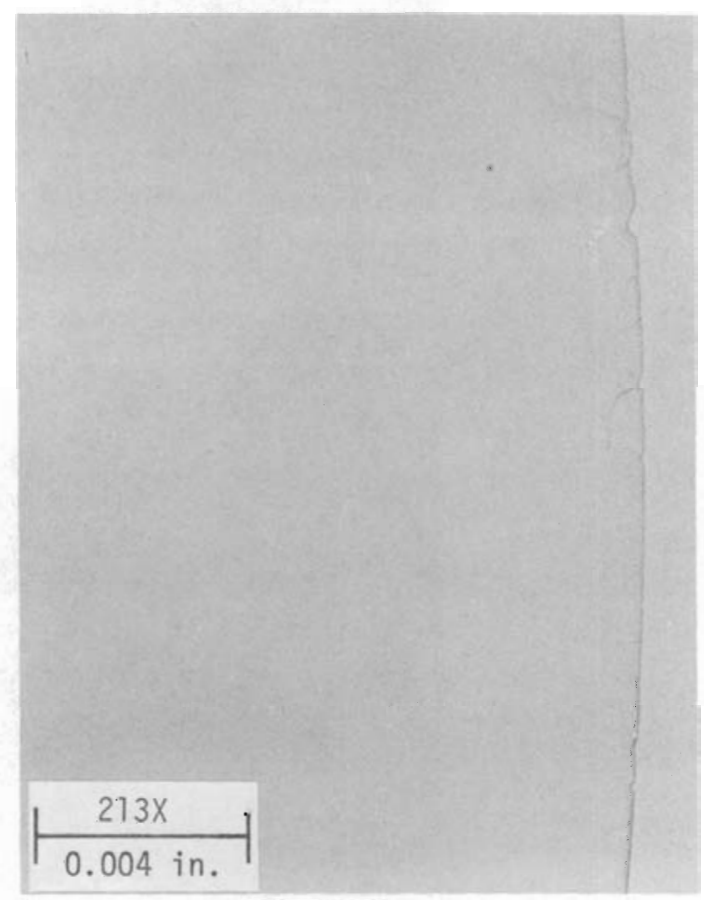

(d) STATIC TEST - HANFORD GROUNDWATER, 43 d AT $150^{\circ} \mathrm{C}$

FIGURE 9. Micrographs of Vitreous Silica Specimens Before and After Leaching 


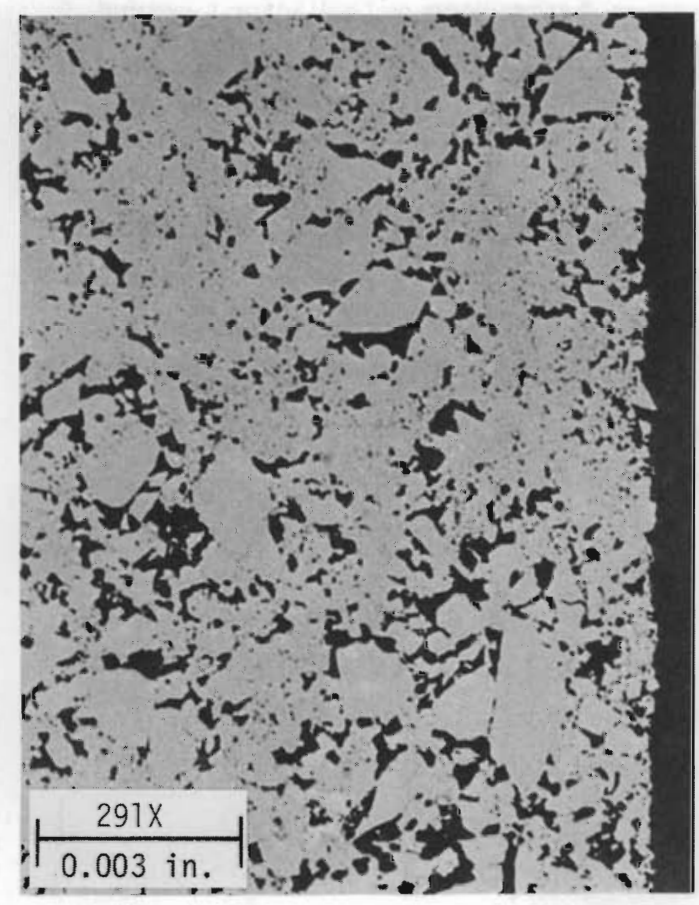

(a) AS-FABRICATED

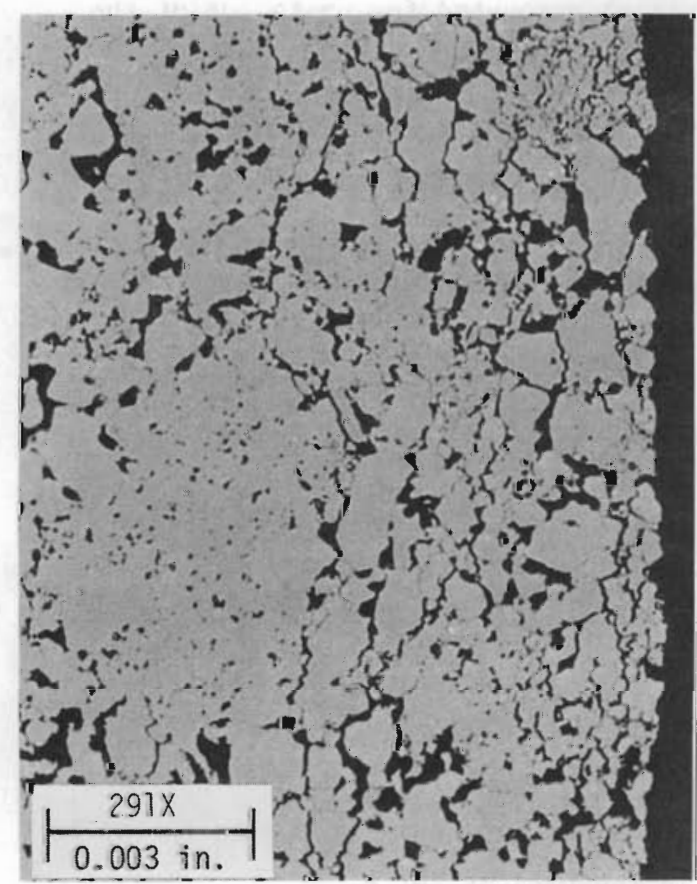

(c) STATIC TEST - DEMINERALIZED WATER, 45 d AT $250^{\circ} \mathrm{C}$

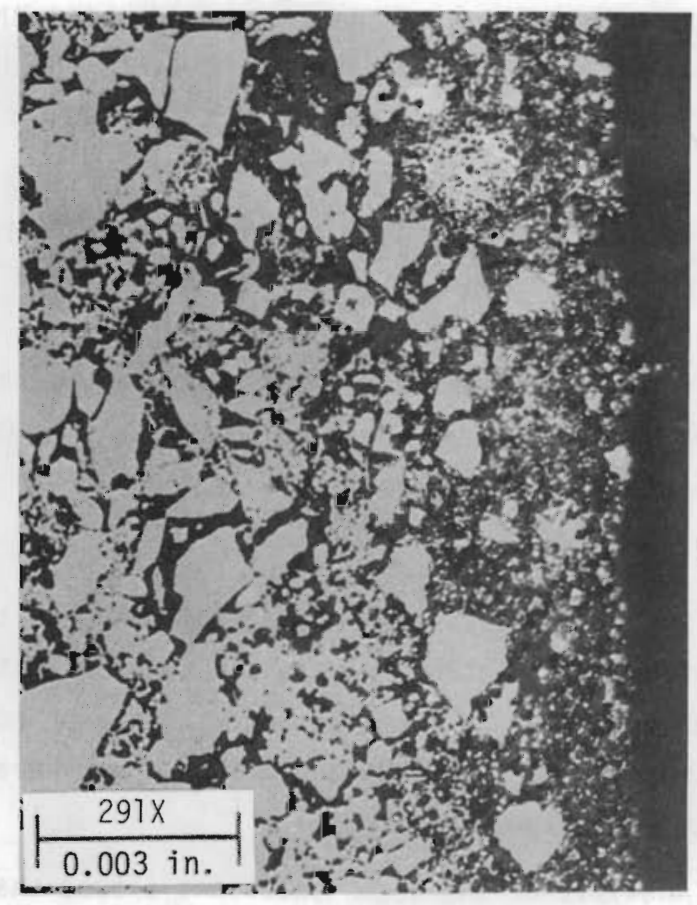

(b) FLOWING DEMINERALIZED WATER, 45 d AT $250^{\circ} \mathrm{C}$

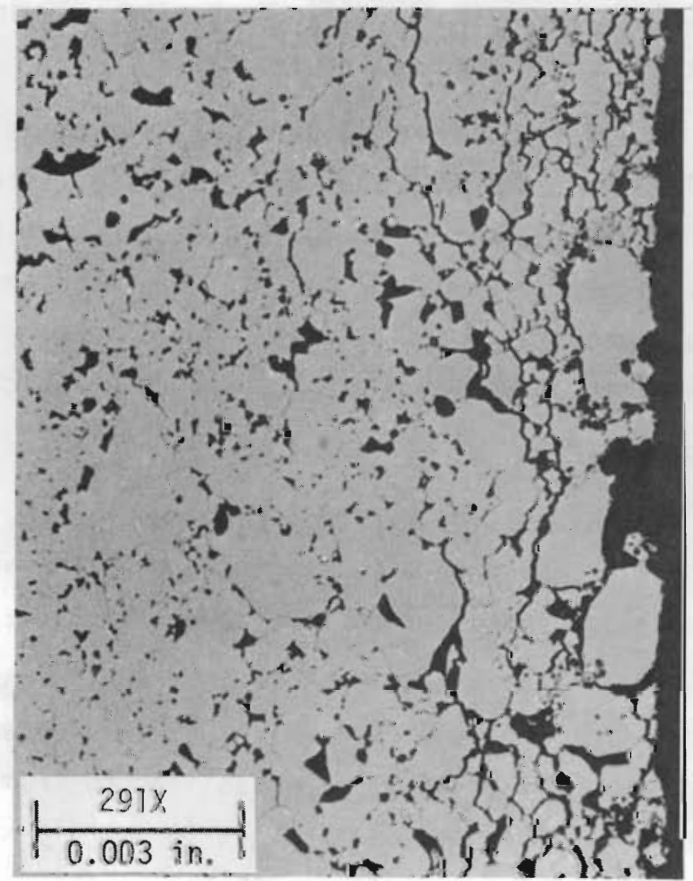

(d) STATIC TEST - BRINE SOLUTION, 45 d AT $250^{\circ} \mathrm{C}$

FIGURE 10. $\mathrm{CaTiO}_{3}$ Specimens Before and After Leaching 
Analysis of the leach solutions showed that calcium was the principal species leached from $\mathrm{CaTiO}_{3}$, with calcium concentrations reaching $300 \mathrm{ppm}$ in some tests done with Hanford ground water. Titanium concentrations were very low in all of the leach solutions, amounting to no more than 1 to $2 \mathrm{ppm}$. Impurities accounted for as much as $40 \%$ of the material leached from the $\mathrm{CaTiO}_{3}$ samples in some tests.

The $\mathrm{CaTiO}_{3}$ specimens used in this work were prepared by hot-pressing at $1600^{\circ} \mathrm{C}$; even so, the specimens exhibited considerable porosity. Decreasing the porosity of the $\mathrm{CaTiO}_{3} \mathrm{Should}$ increase the leach resistance. Unfortunately, there is little information available on techniques for preparing large, dense impervious shapes of $\mathrm{CaTiO}_{3}$. The limited data available on the mechanical and physical properties of $\mathrm{CaTiO}_{3}$ indicate that for the barrier application the properties of $\mathrm{CaTiO}_{3}$ are generally inferior to those of $\mathrm{Al}_{2} \mathrm{O}_{3}$ and graphite.

\section{$4.3 .8 \quad \mathrm{CaZrO}_{3}$}

The leach resistance of $\mathrm{CaZrO}_{3}$ was lower than that of most of the materials tested, and it suffered alteration reactions when exposed to the leach solutions at temperatures of $150^{\circ} \mathrm{C}$ and above $\left(\mathrm{CaZrO}_{3}\right.$ was not tested with the brine solution). Figure 11 shows micrographs of specimens leached at $150^{\circ}$ and $250^{\circ} \mathrm{C}$ in demineralized water and Hanford ground water, and the attack is obvious.

Analysis of the leach solutions showed that calcium was the principal species leached from the $\mathrm{CaZrO}_{3}$, with the calcium content of some solutions being as high as 400 ppm. The zirconium content of the solutions was very low--usually less than $0.5 \mathrm{ppm}$. Leaching of impurities from the $\mathrm{CaZrO}_{3}$ (principally silicon, barium, aluminum, and iron) accounted for up to $10 \%$ of the weight loss suffered by the samples.

Only a limited amount of data are available on the physical and mechanical properties of $\mathrm{CaZrO}_{3}$. From the data that are available, there is nothing that recommends the use of $\mathrm{CaZrO}_{3}$ as a barrier element over the more leach-resistant materials such as $\mathrm{Al}_{2} \mathrm{O}_{3}$. Attempts to hotpress high-density $\mathrm{CaZrO}_{3}$ pellets were unsuccessful and only moderate densities were achieved by cold-pressing and sintering. There is very little information available in the literature on fabricating dense impervious shapes of $\mathrm{CaZrO}_{3}$.

\subsection{9 $\underline{\mathrm{CaTiSiO}}_{5}$}

On the basis of overall leach resistance, $\mathrm{CaTiSiO}_{5}$ was one of the poorer materials tested. In addition, surface alteration and cracking was evident in the specimen exposed to flowing demineralized water at $250^{\circ} \mathrm{C}$ (Figure 12). The $\mathrm{CaTiSiO}$ was not tested with the brine solution.

Analysis of the leach solutions showed that at $250^{\circ} \mathrm{C}$ calcium was the principal species leached from the $\mathrm{CaTiSiO}_{5}$, while the silicon and titanium concentrations were $10 \mathrm{w}$. At $150^{\circ} \mathrm{C}$ silicon was the principal species leached from the CaTiSi0 ${ }_{5}$, and the titanium and calcium concentrations were low. Impurities (primarily sodium, barium and aluminum) accounted for up to $30 \%$ of the material leached from the $\mathrm{CaTiSiO}_{5}$ species in the various tests.

Little data are available on the physical and mechanical properties of $\mathrm{CaTiSi0}_{5}$, and procedures for fabricating large, dense impervious shapes have not been developed. The specimens 


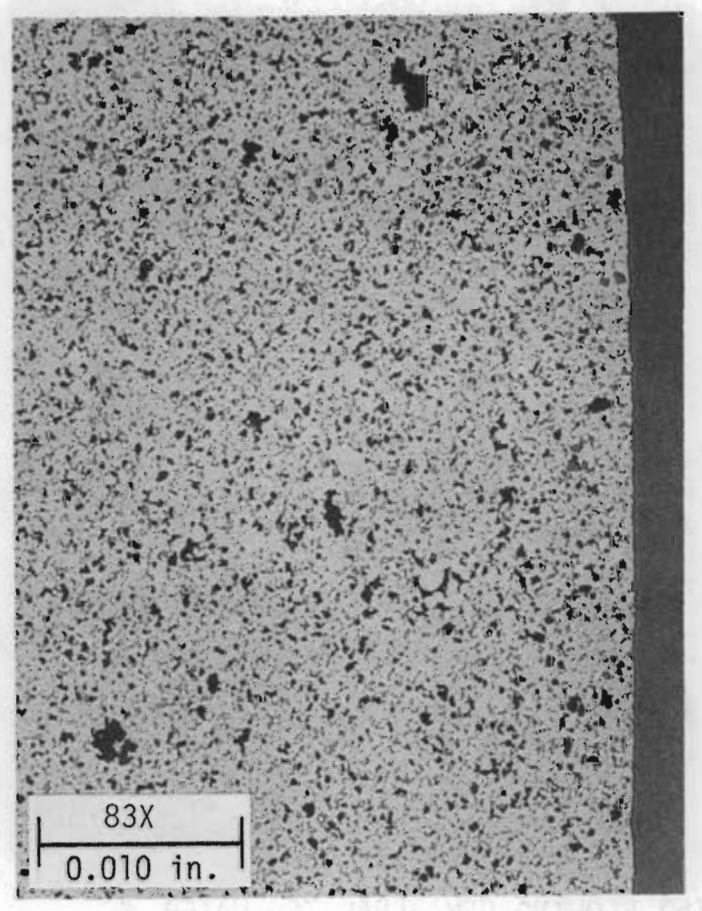

(a) AS-FABRICATED

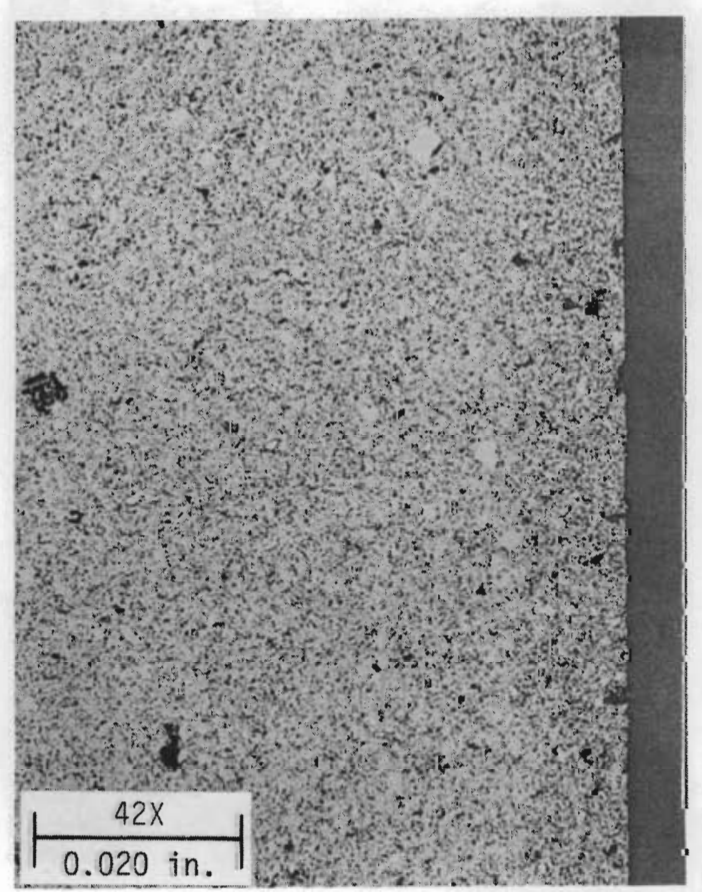

(c) STATIC TEST - DEMINERALIZED WATER, 45 d AT $250^{\circ} \mathrm{C}$

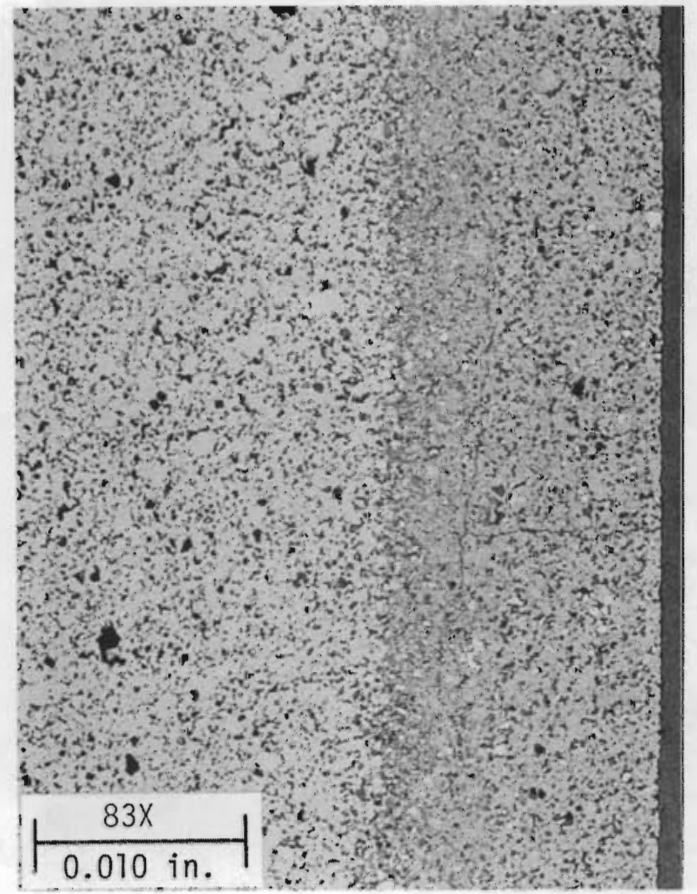

(b) FLOWING DEMINERALIZED WATER, 45 d AT $250^{\circ} \mathrm{C}$

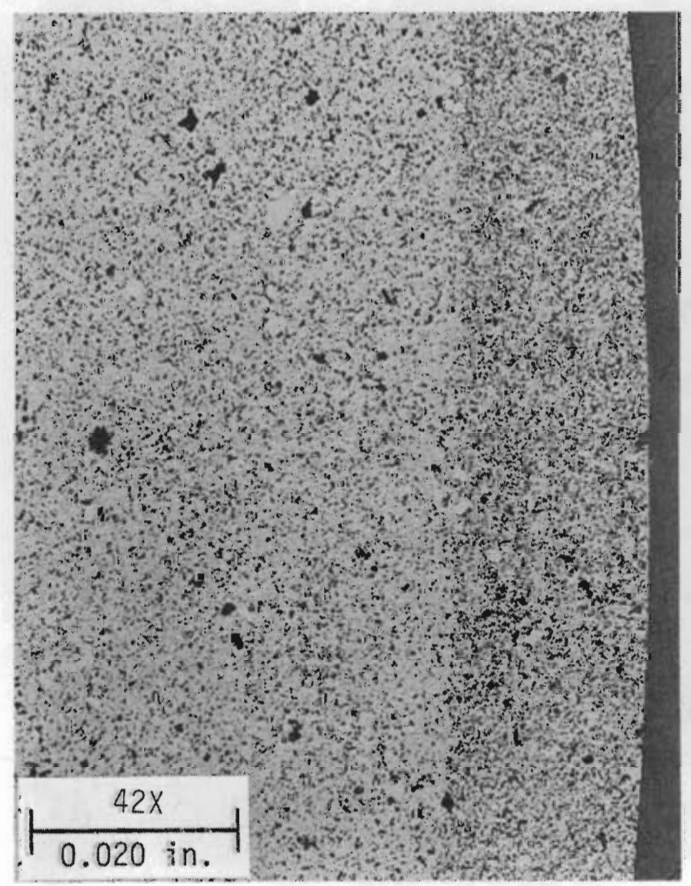

(d) STATIC TEST - DEMINERAL IZED WATER, 43 dे AT $150^{\circ} \mathrm{C}$

FIGURE 11. $\mathrm{CaZrO}_{3}$ Specimens Before and After Leaching 


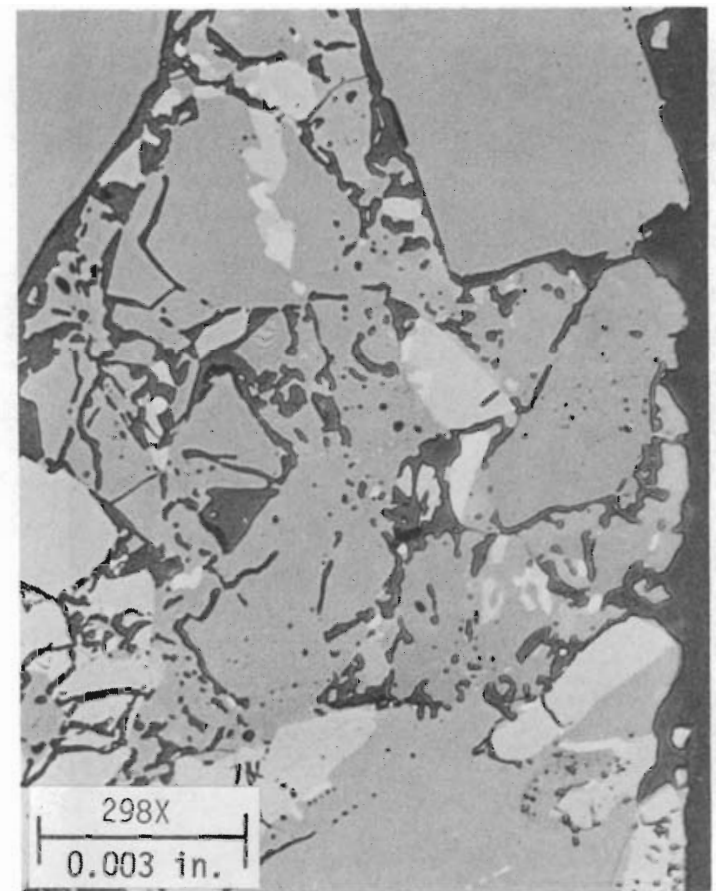

(a) AS-FABRICATED

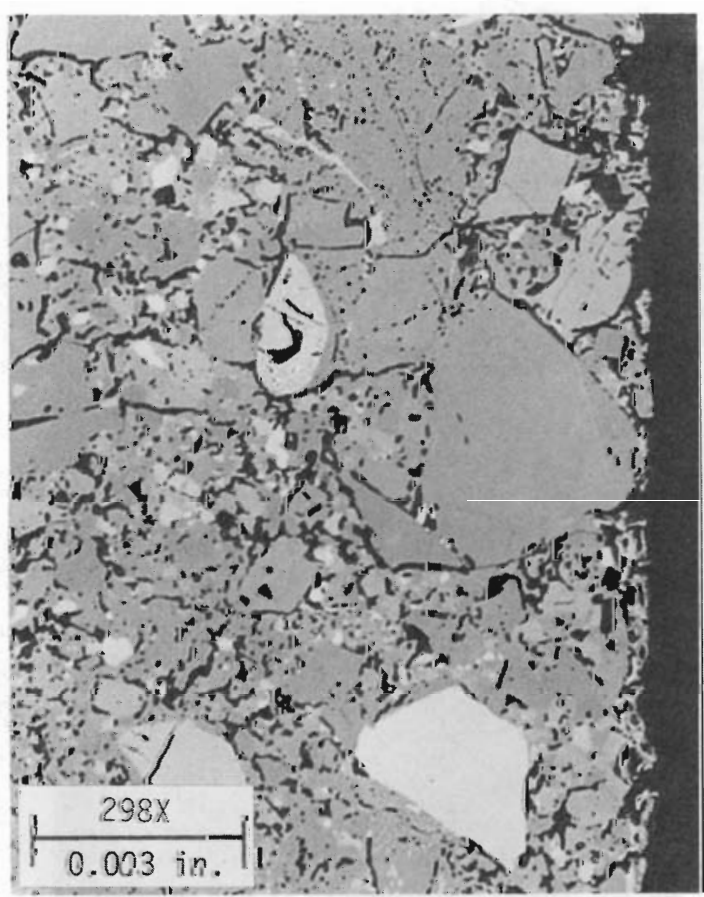

(c) STATIC TEST - DEMINERALIZED WATER, 45 d AT $250^{\circ} \mathrm{C}$

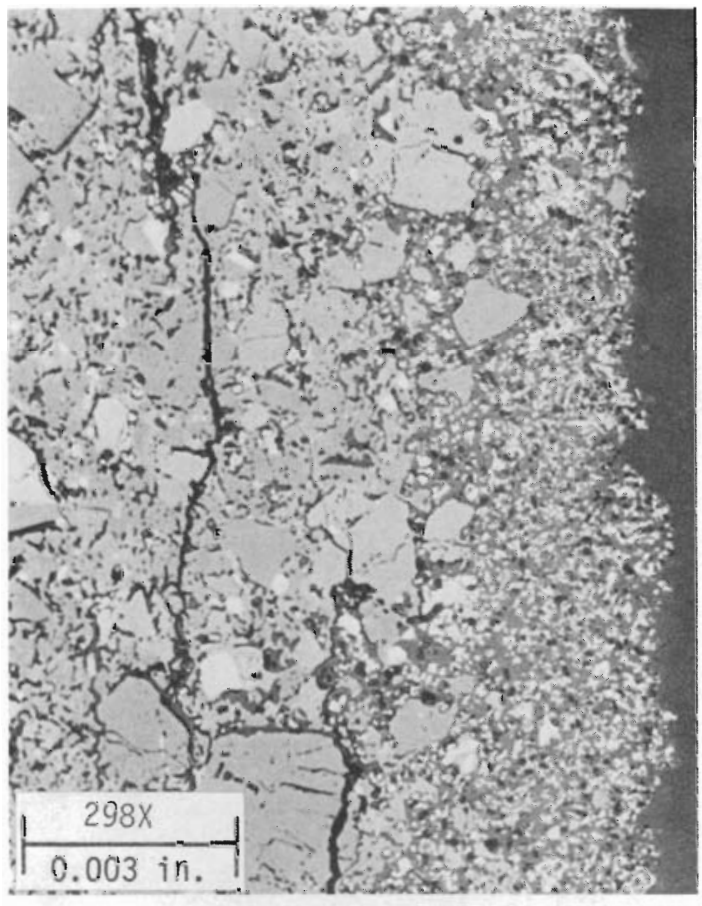

(b) FLOWING DEMINERALIZED WATER, 45 d AT $250^{\circ} \mathrm{C}$

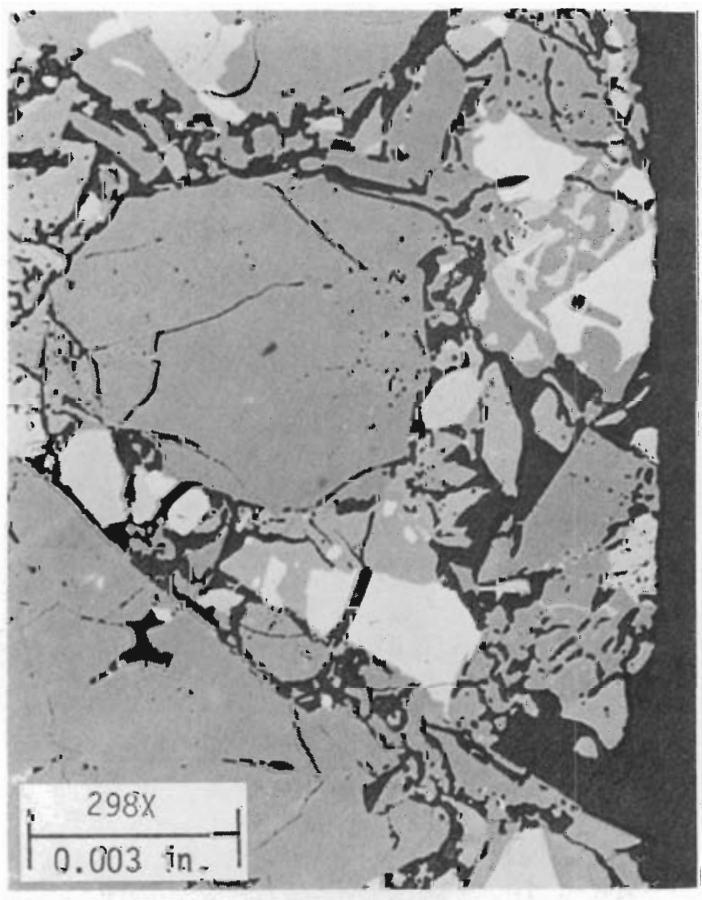

(d) STATIC TEST - GROUNDWATER, 45 d AT $250^{\circ} \mathrm{C}$

FIGURE 12. Micrographs of $\mathrm{CaTiSiO}_{5}$ Specimens Before and After Leaching 
used in this work were prepared by cold-pressing and sintering and had a relatively low density. Attempts to hot-press $\mathrm{CaTiSiO}_{5}$ were unsuccessful. Limited quantities of relatively pure $\mathrm{CaTiSiO}_{5}$ are available as the naturally occurring mineral sphene.

\subsubsection{Basalt}

The leach resistance of basalt was inferior to that of several of the other materials tested, and exposure to the leach solutions at high temperatures produced alteration reactions (the basalt was not tested with the brine solution). The alteration reactions appeared to begin at about $150^{\circ} \mathrm{C}$ and were quite rapid at $250^{\circ} \mathrm{C}$ (Figure 13 ).

Analys is of the leach solutions from the static tests showed that silicon was the principal cation species leached from the basait, together with smaller amounts of sodium, potassium, and aluminum. The analytical data from the $250^{\circ} \mathrm{C}$ static tests showed that the amount of material dissolved from a given sample was substantially greater than the sample's measured weight loss, indicating that some form of hydration reaction(s) had probabiy occurred at the sample surface.

Basalt has two potential advantages as a barrier material: 1) it is inexpensive and readily available, and 2) if a basalt repository is developed, it could be advantageous to have a barrier element fabricated from the same material as the repository medium. Disadvantages, in addition to poor leach resistance, include: 1) lack of demonstrated technology for fabricating large, dense impervious shapes, and 2) mechanical and physical properties that are generally inferior to those of graphite and alumina. On an overall basis, there appears to be 1ittle justification for further consideration of basalt as a barrier material.

\subsubsection{1 $\mathrm{ZrSi0}_{4}$}

On an overall basis the leach resistance of $\mathrm{ZrSiO}_{4}$ was lower than that of most of the materials tested. The specimen exposed to flowing demineralized water at $250^{\circ} \mathrm{C}$ suffered an alteration reaction, but the specimens from the static tests at $250^{\circ} \mathrm{C}$ showed little evidence of attack by the leach solutions (Figure 14).

Analyses of the leach solutions showed that silicon was the principal species leached from the $\mathrm{ZrSiO}_{4}$. The zirconium content of the leach solutions was very low. Small amounts of sodium and calcium were also leached from the $\mathrm{ZrSiO}_{4}$.

In general, the pertinent mechanical and physical properties of $\mathrm{ZrSiO}_{4}$ are inferior to those of $\mathrm{Al}_{2} \mathrm{O}_{3}$, with two exceptions. The impact strength of $\mathrm{ZrSiO}_{4}$ is much greater than that of $\mathrm{Al}_{2} \mathrm{O}_{3}$, and its thermal expansion coefficient is lower. Dense shapes of $\mathrm{ZrSiO}_{4}$ are reported to have poor thermal-shock resistance.

Examination of the micrographs in Figure 14 point out an additional problem with $\mathrm{ZrSiO}_{4}$. The test specimens used in this study were prepared by hot-pressing at $\sim 1600^{\circ} \mathrm{C}$. Even so, the density of the specimens was low and they exhibited considerable continuous porosity. Zirconium silicate is reported to decompose at about $1675^{\circ} \mathrm{C}$, which prevents hot-pressing at temperatures much above those used in this work. The limited information available in the literature indicates it is difficult to produce high-density shapes from $\mathrm{ZrSiO}_{4}$. 


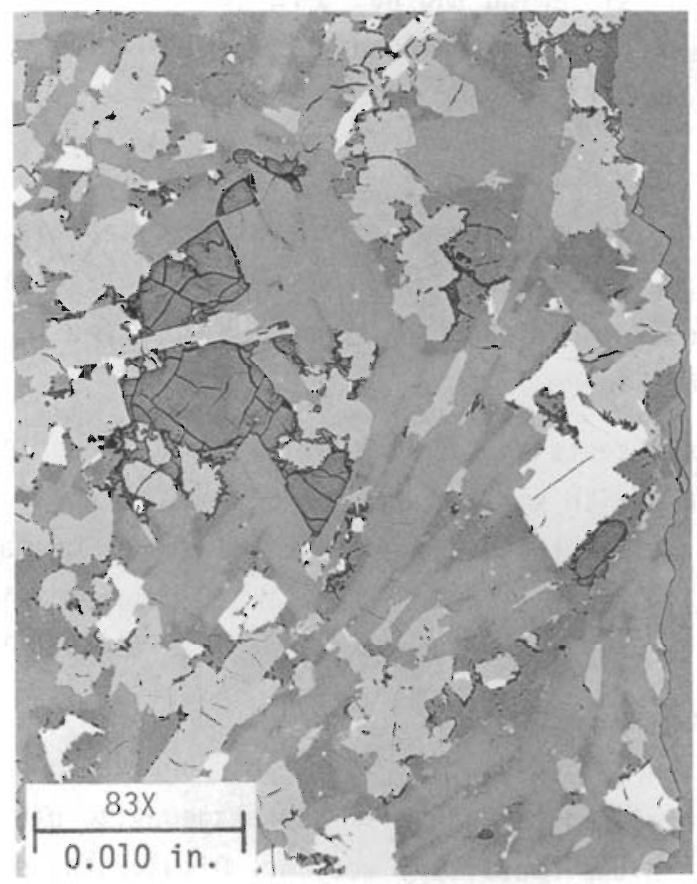

(a) AS-RECEIVED

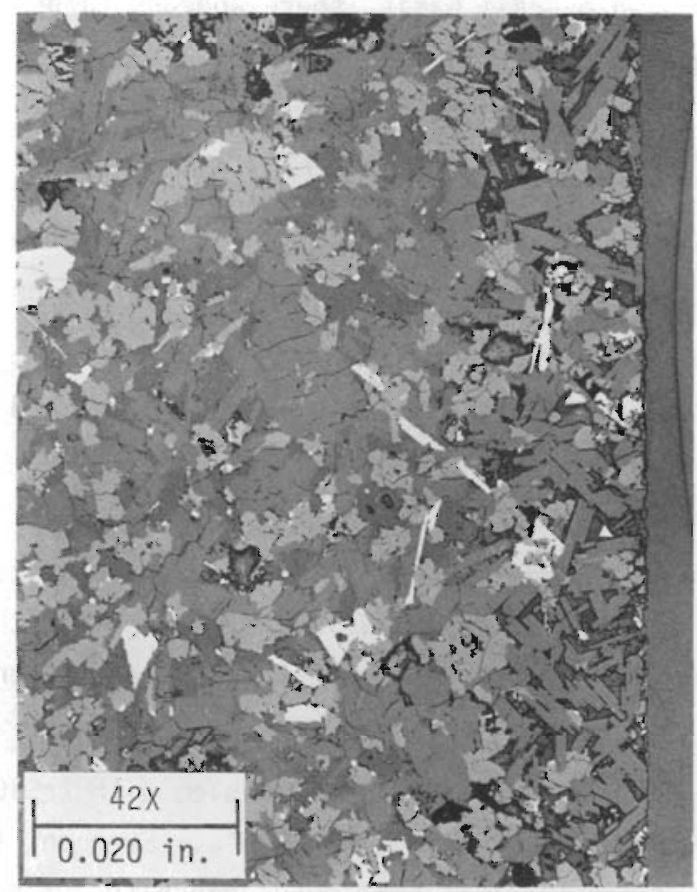

(c) STATIC TEST - DEMINERALIZED WATER, 45 d AT $250^{\circ} \mathrm{C}$

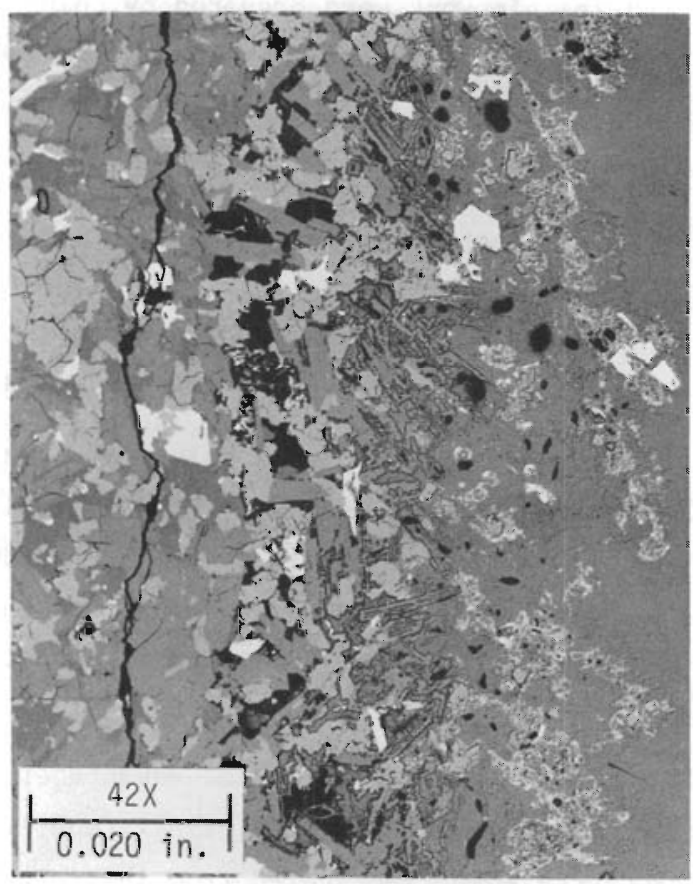

(b) FLOWING DEMINERALIZED WATER, 45 d AT $250^{\circ} \mathrm{C}$

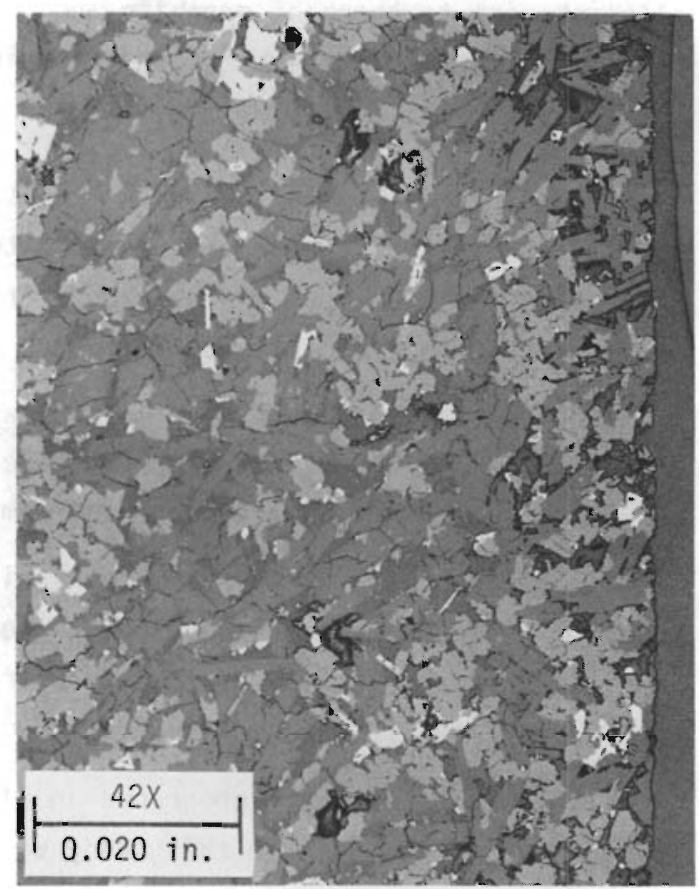

(d) STATIC TEST - GROUNDWATER, 45 d AT $250^{\circ} \mathrm{C}$

FIGURE 13. Basalt Specimens Before and After Testing 


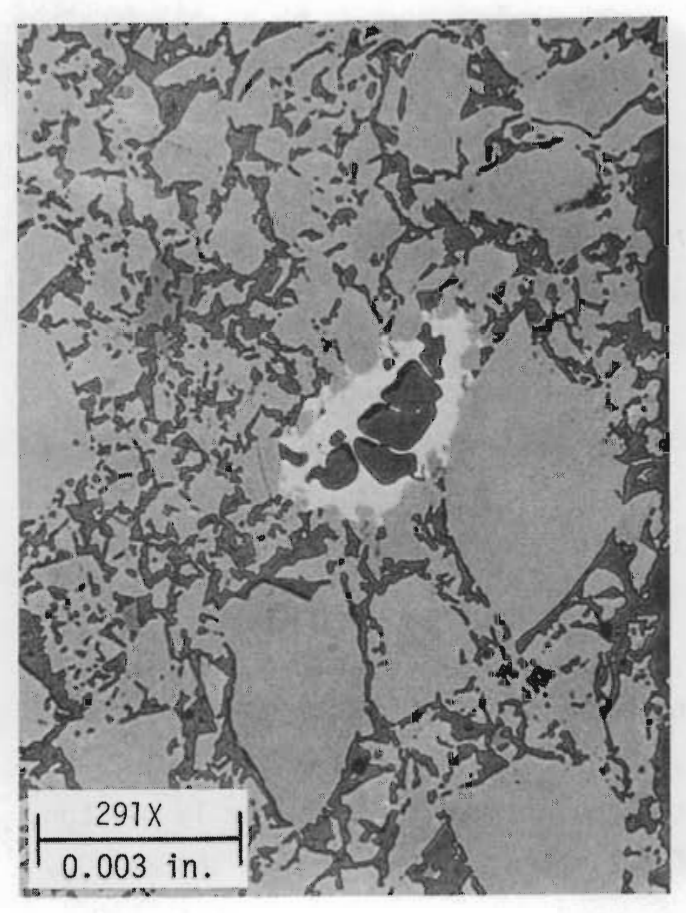

(a) AS-FABRICATED

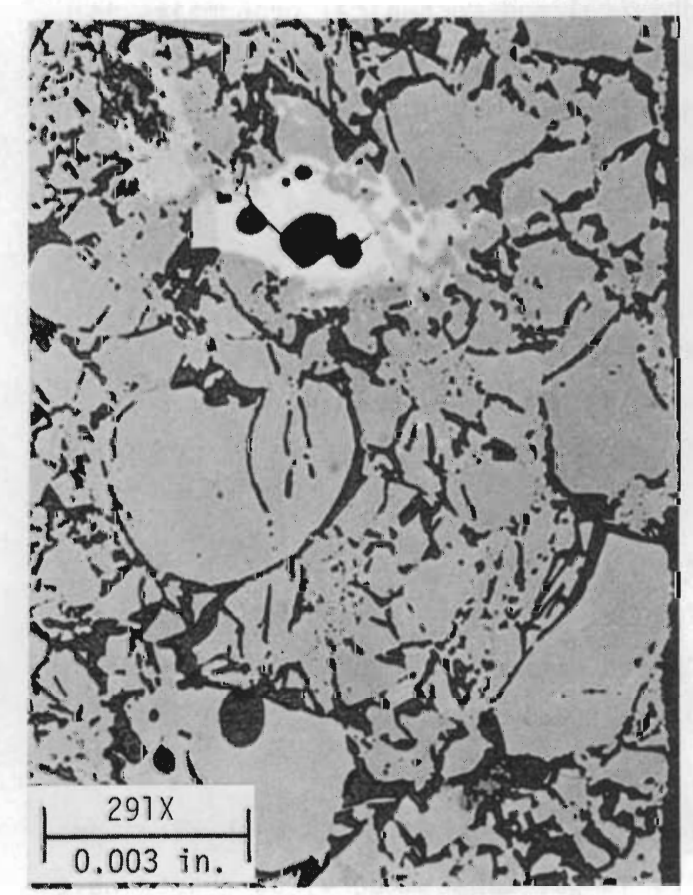

(c) STATIC TEST - DEMINERALIZED WATER, $45 \mathrm{~d}$ AT $250^{\circ} \mathrm{C}$

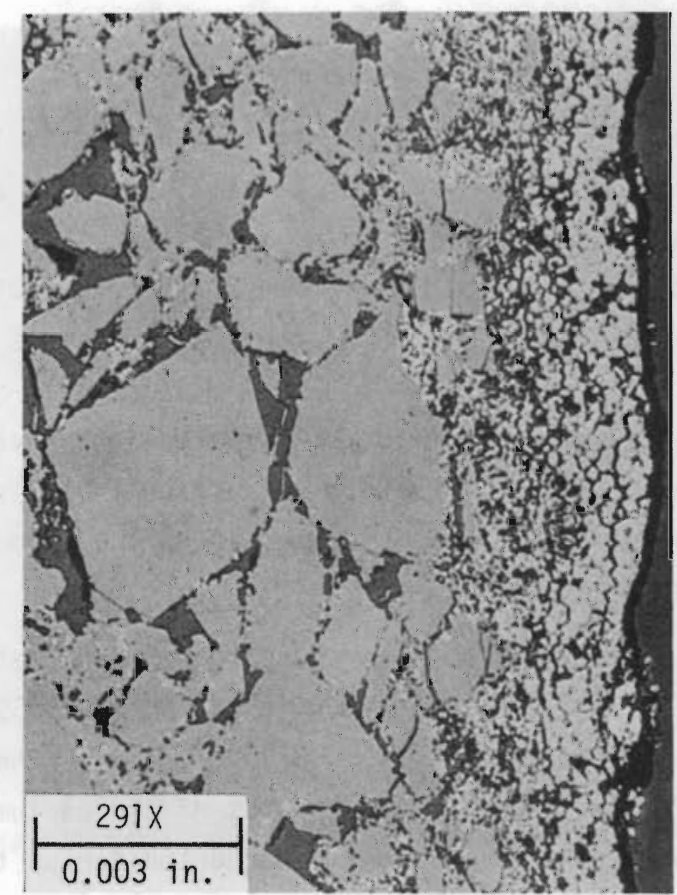

(b) FLOWING DEMINERALIZED WATER, 45 d AT $2500 \mathrm{C}$

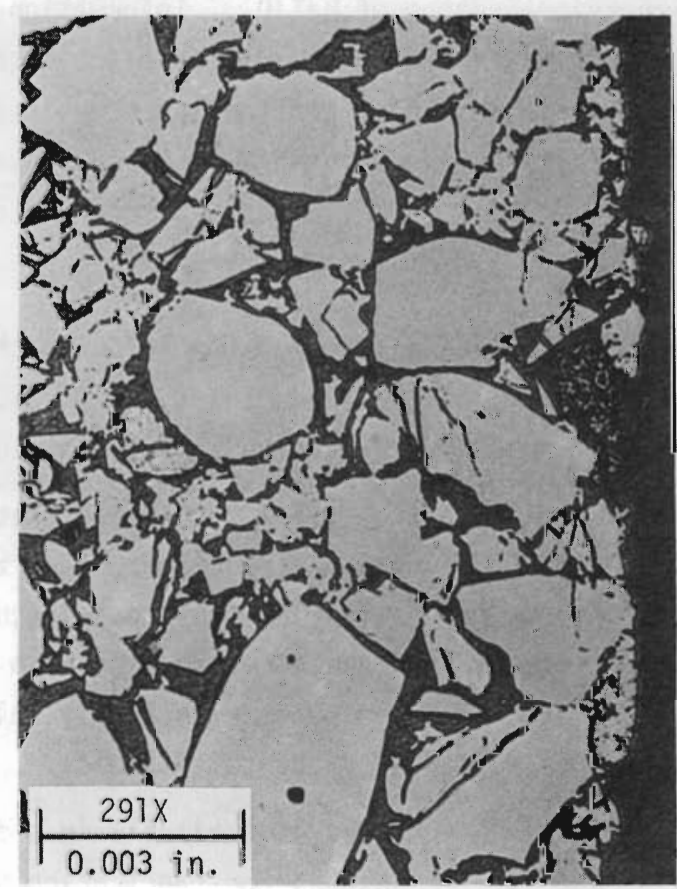

(d) STATIC TEST - BRINE SOLUTION, $45 \mathrm{~d}$ AT $250^{\circ} \mathrm{C}$

FIGURE 14. Photomicrographs of $\mathrm{ZrSiO}_{4}$ Specimens 
Zirconium silicate is available in limited amounts as the naturally occurring mineral zircon.

\subsubsection{2 $\underline{\mathrm{BaTiO}}_{3}$}

The leaching data indicate that $\mathrm{BaTiO}_{3}$ is less resistant to leaching than are most of the materials tested. In the static tests at $150^{\circ}$ and $250^{\circ} \mathrm{C}$, however, there were large differences between the $\mathrm{BaTiO}_{3}$ leach rates calculated from weight loss measurements and those determined from solution analyses. In every case the solution analyses gave much lower leach rates. Ceramographic examination of the test specimens indicated that alteration reactions did not occur to any extent even at $250^{\circ} \mathrm{C}$ (Figure 15). Most of the specimens tested at $150^{\circ}$ and $250^{\circ} \mathrm{C}$ were either cracked or shattered into several pieces. The micrographs in Figure 15 show that the test specimens were very porous. It is possible that the high leach rates calculated from the weight loss measurements resulted from the spalling of small fragments from the specimen surface.

Analysis of the leach solutions showed that barium was the principal species leached from the $\mathrm{BaTiO}_{3}$. In the static tests at $150^{\circ}$ and $250^{\circ} \mathrm{C}$ the barium concentration appeared to reach a steady-state level very quickly ( $\sim 1$ day). The titanium concentration in the leach solution was very low--usually less than $5 \%$ of the barium concentration. Impurities (primarily silicon, calcium, aluminum and sodium) accounted for up to $20 \%$ of the material leached from the $\mathrm{BaTiO}_{3}$.

Attempts to hot-press $\mathrm{BaTiO}_{3}$ were unsuccessfui and pellets prepared by cold-pressing and sintering were very porous. There is little data in the literature on methods for preparing dense impervious shapes of $\mathrm{BaTiO}_{3}$. Information on the physical and mechanical properties is limited, and the data that is available indicates that $\mathrm{BaTiO}_{3}$ is generally inferior to $\mathrm{Al}_{2} \mathrm{O}_{3}$ and $\mathrm{TiO}_{2}$. It is possible that the leach resistance of dense impervious $\mathrm{BaTiO}_{3}$ would be lower than the results obtained in this study, but even so there is little incentive to give additional consideration to $\mathrm{BaTiO}_{3}$ as a barrier material.

\subsubsection{3 $\mathrm{BaZrO}_{3}$}

Barium zirconate was one of the least leach-resistant materials tested. In addition, exposure of $\mathrm{BaZrO}_{3}$ to the leach solutions at $250^{\circ} \mathrm{C}$ produced extensive alteration reactions (Figure 16). Most of the specimens tested at $150^{\circ}$ and $250^{\circ} \mathrm{C}$ suffered some degree of cracking.

Analyses of the leach solutions showed that barium was the principal species leached from the $\mathrm{BaZrO}_{3}$ (up to $500 \mathrm{ppm}$ ). Zirconium concentrations were much lower (up to 100 ppm), but were still much higher than in any of the other leach tests involving ceramic materials containing zirconium. Reasons for the increased zirconium concentrations were not determined. Impurities leached from the specimens, principally calcium and silicon, accounted for up to $20 \%$ of the weight loss suffered by the test samples.

Very little information is available in the literature on the mechanical and physical properties of $\mathrm{BaZrO}_{3}$. Techniques for fabricating $\mathrm{BaZrO}_{3}$ into large, dense impervious shapes have not been developed. Based on its poor leach resistance and the lack of needed property data, $\mathrm{BaZrO}_{3}$ is dropped from further consideration as a barrier material. 


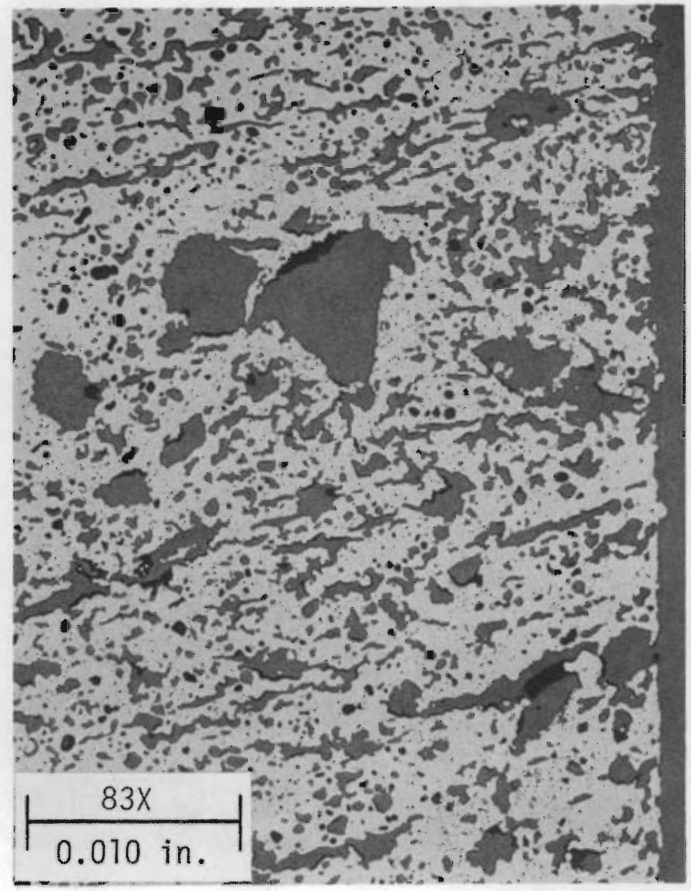

(a) AS-FABRICATED

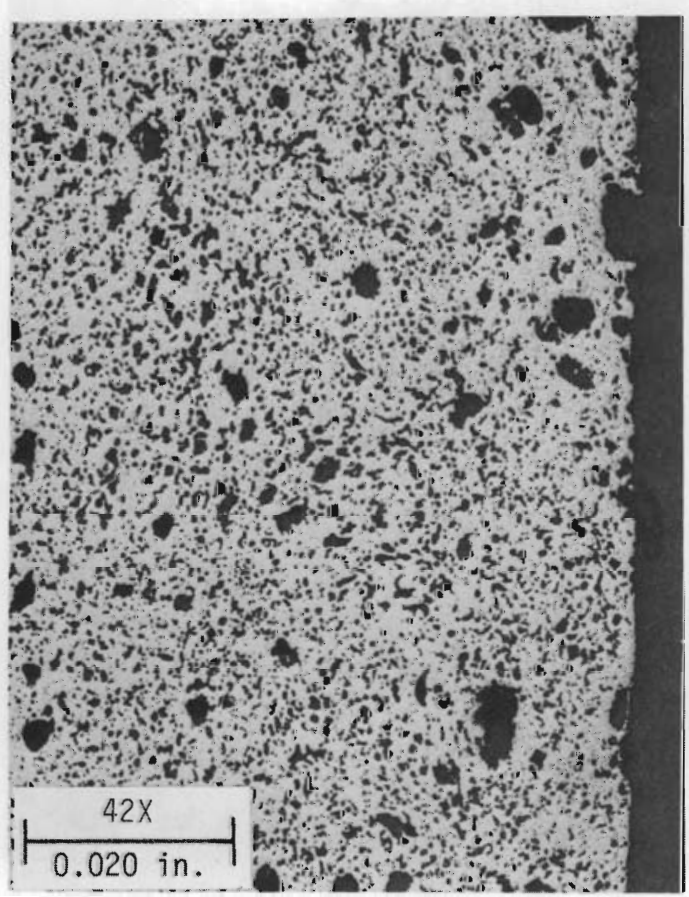

(c) STATIC TEST - BRINE SOLUTION, 45 d AT $250^{\circ} \mathrm{C}$

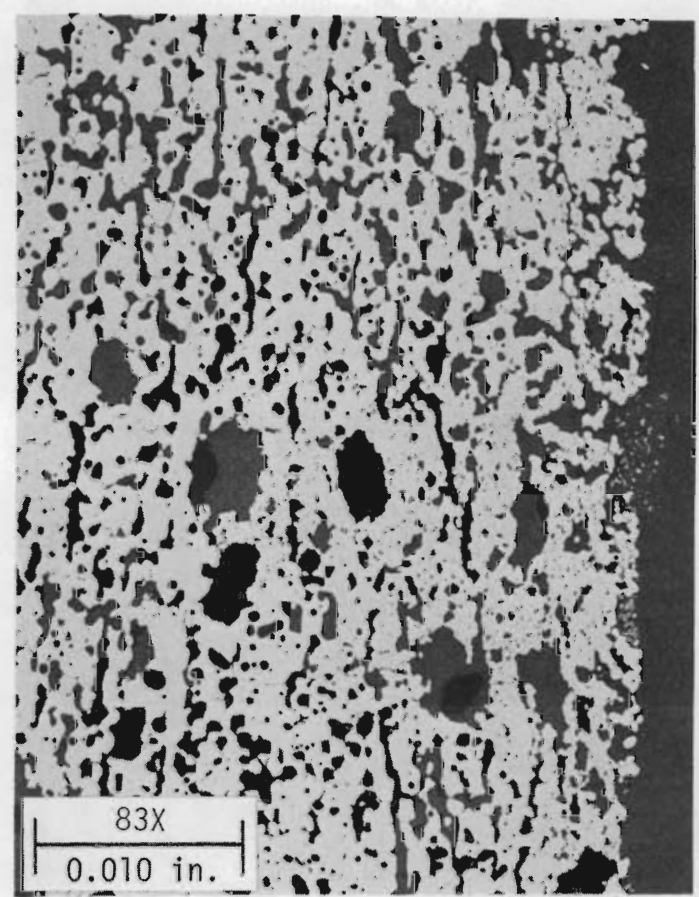

(b) FLOWING DEMINERALIZED WATER, 45 d AT $250^{\circ} \mathrm{C}$ 


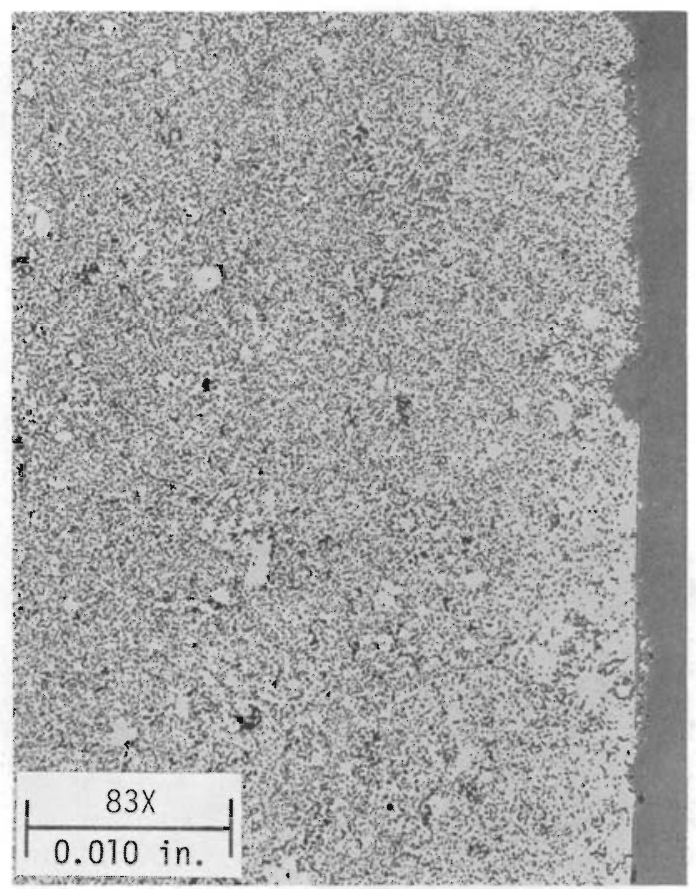

(a) AS-PREPARED

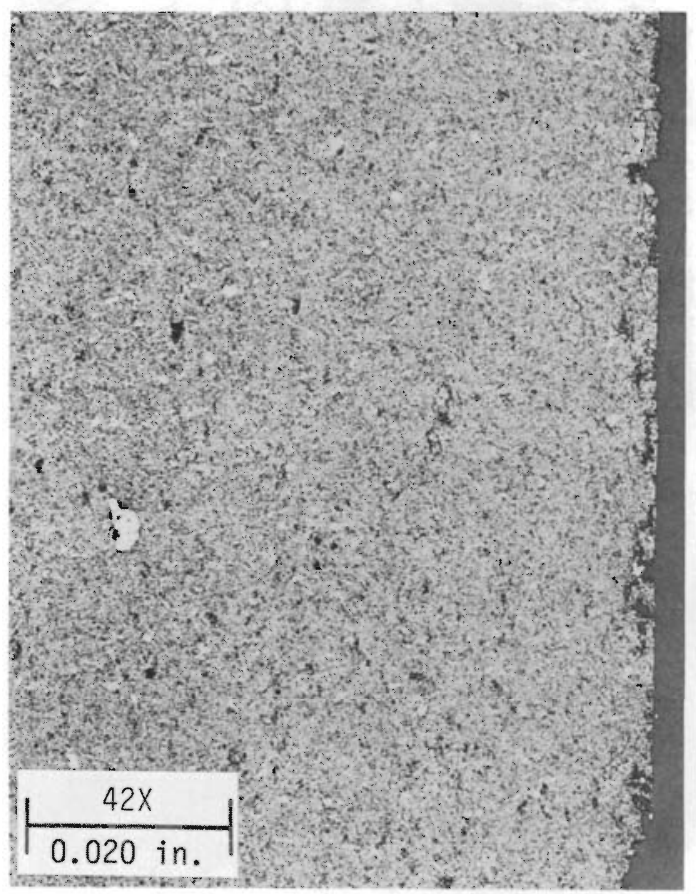

(c) STATIC TEST - DEMINERALIZED WATER, 45 d AT $250^{\circ} \mathrm{C}$

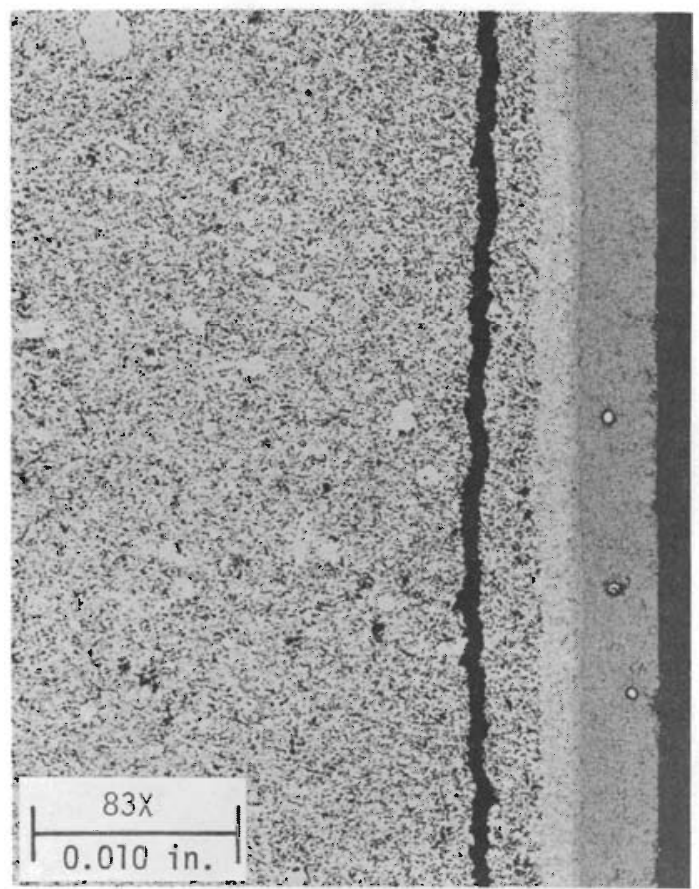

(b) FLOWING DEMINERALIZED WATER, 45 d AT $250^{\circ} \mathrm{C}$

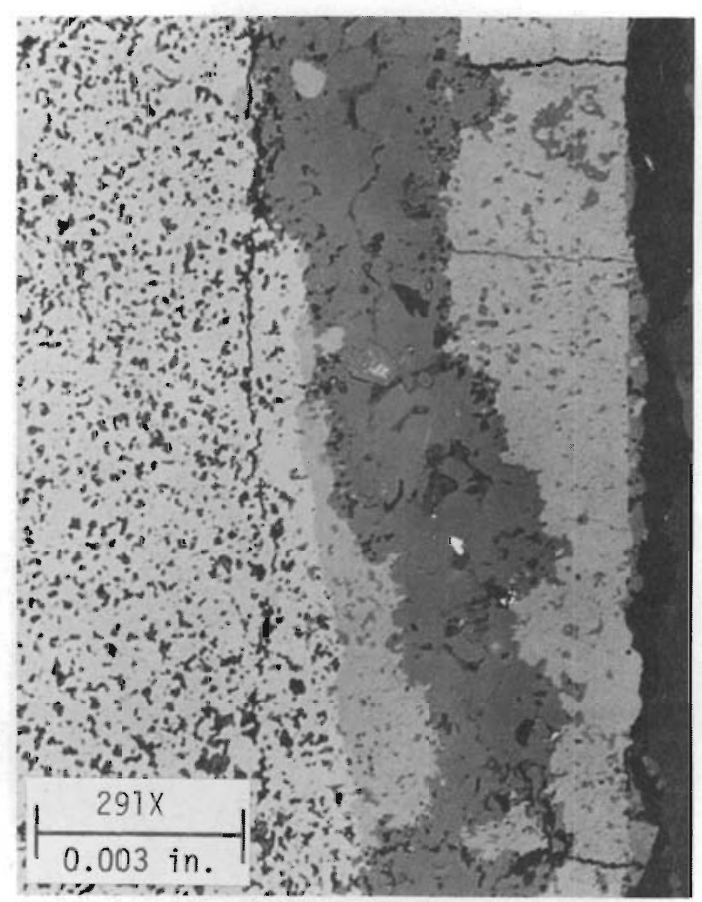

(d) STATIC TEST - BRINE SOLUTION, 45 d AT $250^{\circ} \mathrm{C}$

FIGURE 16. Micrographs of $\mathrm{BaZrO}_{3}$ Specimens Before and After Leaching 


\subsubsection{Pyroceram 9617}

Pyroceram 9617 is a glass-ceramic developed by Corning Glass Works. It can be produced in large, dense impervious shapes and possesses a number of physical and mechanical properties that are desirable in a barrier element. The leach resistance of Pyroceram 9617 is relatively high at low temperatures, but decreases rapidly with increasing temperature. In addition, exposure of the Pyroceram 9617 to the leach solutions produced alteration reactions that appeared to start at temperatures as low as $150^{\circ} \mathrm{C}$ and become extensive at $250^{\circ} \mathrm{C}$ (Figure 17). After a specimen was dried, the reaction layer was powdery and very easily removed from the surface. Analyses of the leach solutions from the static tests at $150^{\circ}$ and $250^{\circ} \mathrm{C}$ showed very high concentrations of dissolved cations, especially silicon, lithium, and aluminum. The high aluminum concentrations (up to $40 \mathrm{ppm}$ ) were surprising because the tests with $\mathrm{Al}_{2} \mathrm{O}_{3}$ indicated that the saturation concentrations for aluminum in the leach solutions were much lower. The high aluminum concentrations observed in the Pyroceram 9617 tests may result from changes in $\mathrm{pH}$ of the leach solutions due to dissolution of various cations in the Pyroceram 9617 (i.e., $\mathrm{Li}, \mathrm{Na}$ ) or from the formation of soluble aluminum complexes.

Based on the experimental results obtained, there appears to be little incentive to consider Pyroceram 9617 for use as an element in an engineered-barrier system. One mitigating factor is the possiblity that the repository might be designed to maintain the maximum temperature below $100^{\circ} \mathrm{C}$. If this should happen, there is some incentive to evaluate Pyroceram 9617 in more detail.

\subsubsection{Marcor 9658}

Marcor 9658 is a machinable glass-ceramic developed by the Corning Glass Works. Marcor possesses a number of desirable properties that could make it a potentially useful barrier material if it had the necessary corrosion resistance. Unfortunately, Marcor rated at or near the bottom in all of the leach tests. In addition to its high leach rates, Marcor underwent alteration reactions when exposed to the leach solutions at $250^{\circ} \mathrm{C}$ (Figure 18). There were also indications that the alteration reactions occurred at temperatures as low as $150^{\circ} \mathrm{C}$ in the Hanford ground water (Figure 18f).

Analysis of the leach solutions from the static tests at $150^{\circ}$ and $250^{\circ} \mathrm{C}$ showed that the principal species leached from the Marcor were very soluble in the leach solutions. Thus, saturation effects in static systems would have less effect on the leaching of Marcor than on most of the other ceramic material tested.

Based on the results of the leach tests, the Marcor can be dropped from further consideration as a barrier material.

\subsection{ESTIMATES OF LONG-TERM ATTACK}

In order for a ceramic barrier element to be of use in a nuclear waste package, it must provide isolation of the primary waste canister from the repository environment for the required 1000-year period. It is impossible to estimate with any degree of accuracy the 


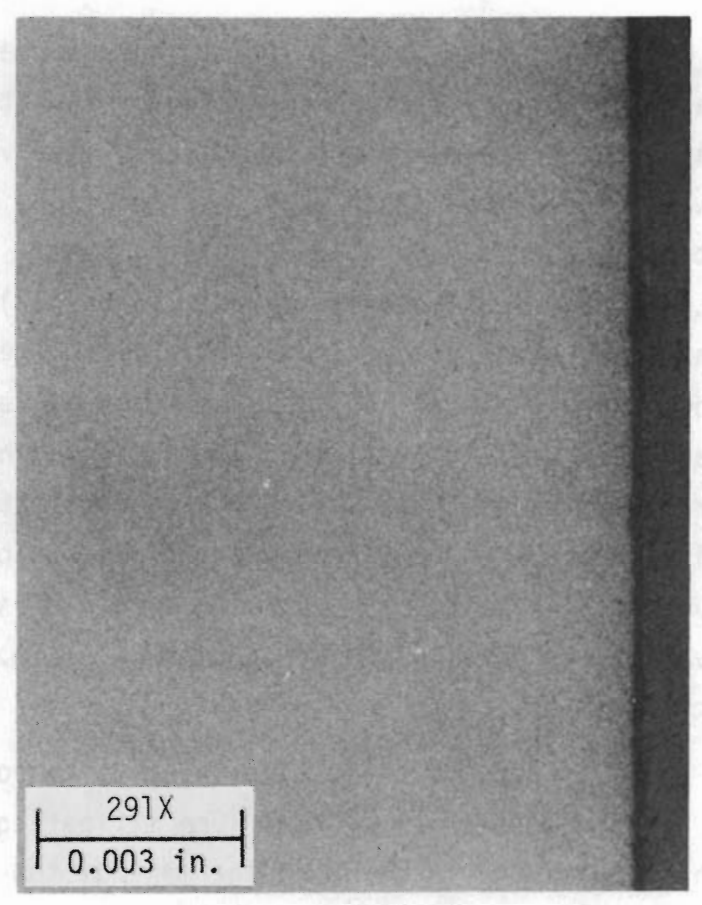

(a) AS-RECEIVED

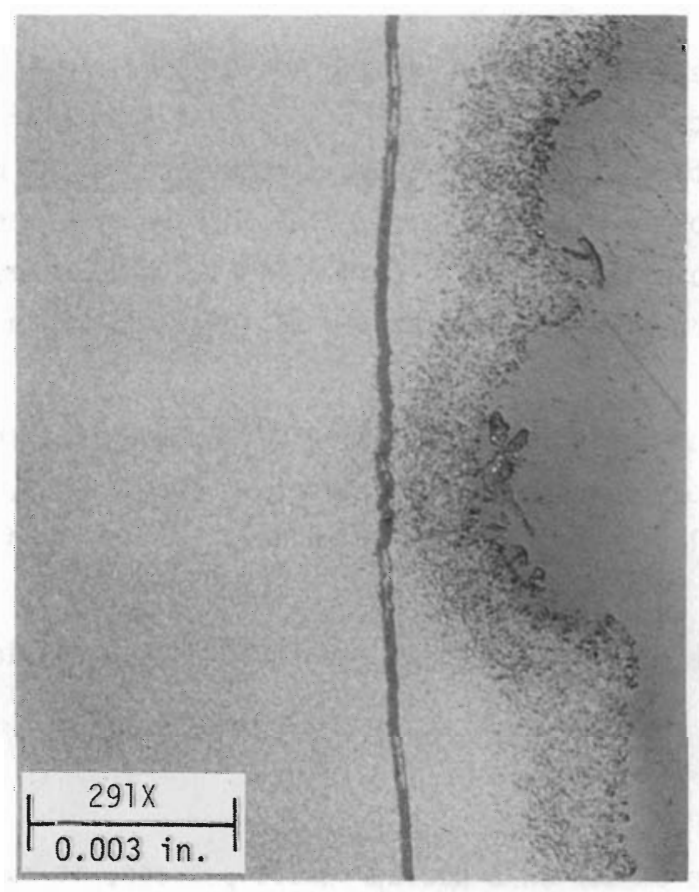

(c) STATIC TEST - GROUNDWATER,
43 d AT $250^{\circ} \mathrm{C}$

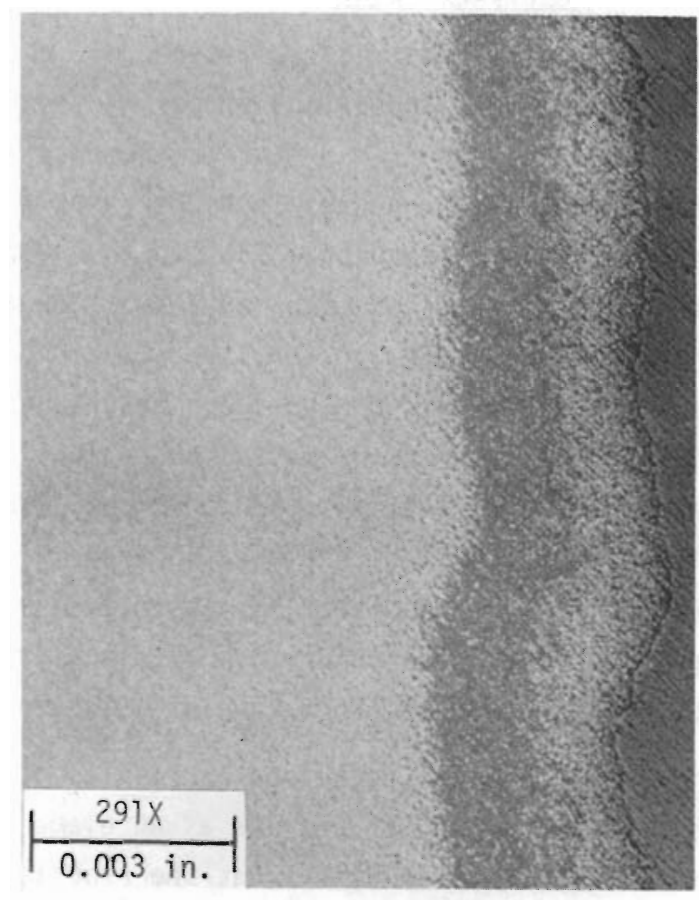

(b) STATIC TEST - DEMINERALIZED WATER, $43 \mathrm{~d}$ AT $250^{\circ} \mathrm{C}$

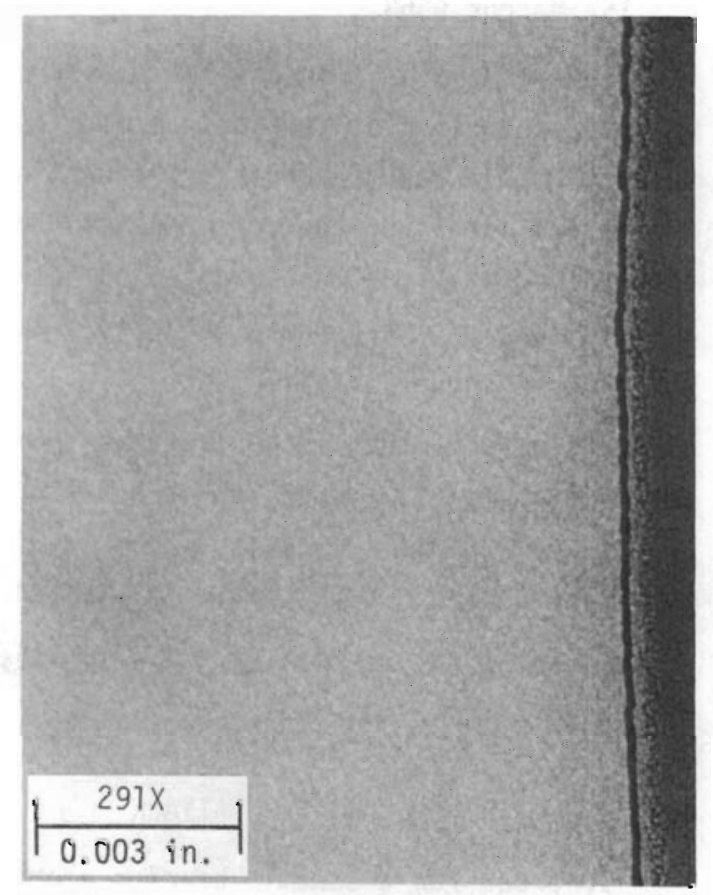
(d) STATIC TEST
43 d AT $250^{\circ} C^{-B R I N E ~ S O L U T I O N, ~}$

FIGURE 17. Micrographs of Pyroceram 9617 Specimens 


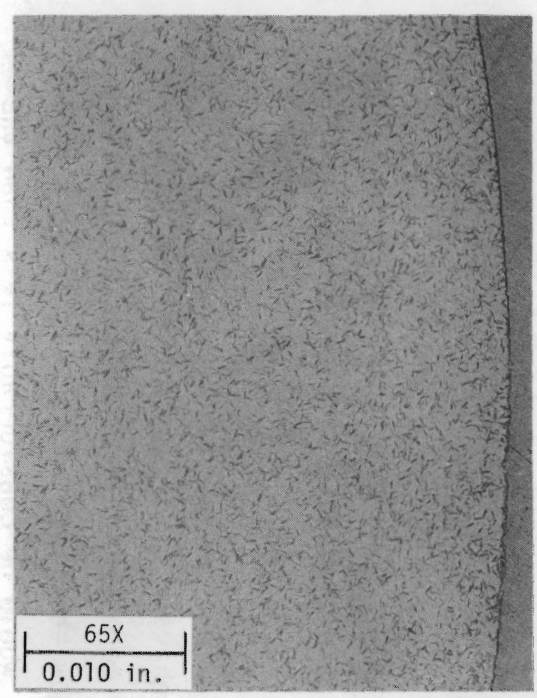

(a) AS-RECEIVED

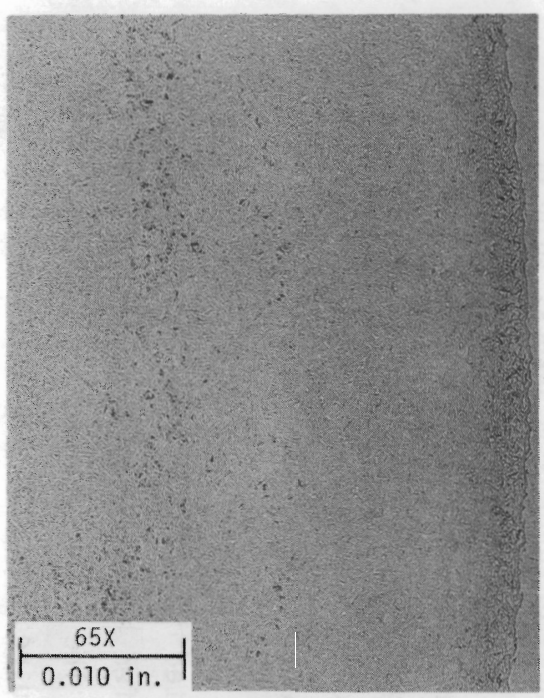

(b) FLOWING DEMINERALIZED WATER,
43 d AT $250^{\circ} \mathrm{C}$

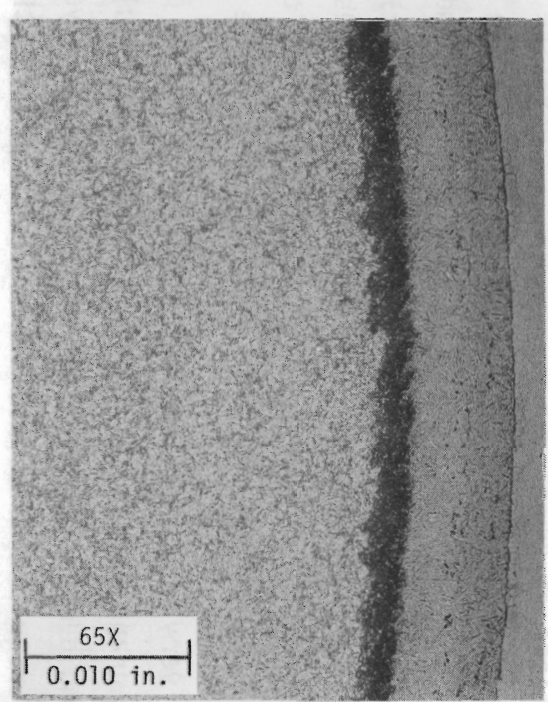

(e) STATIC TEST - BRINE SOLUTION,

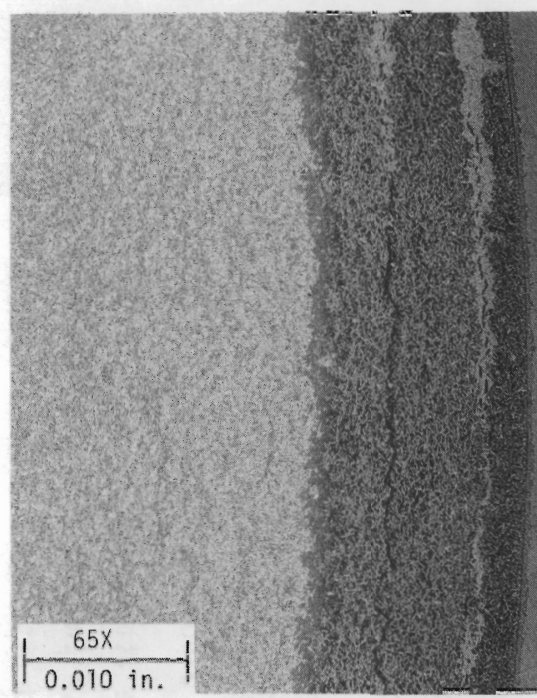
(c) STATIC TEST - DEMINERALIZED WATER,
$43 \mathrm{~d}$ AT $250^{\circ} \mathrm{C}$

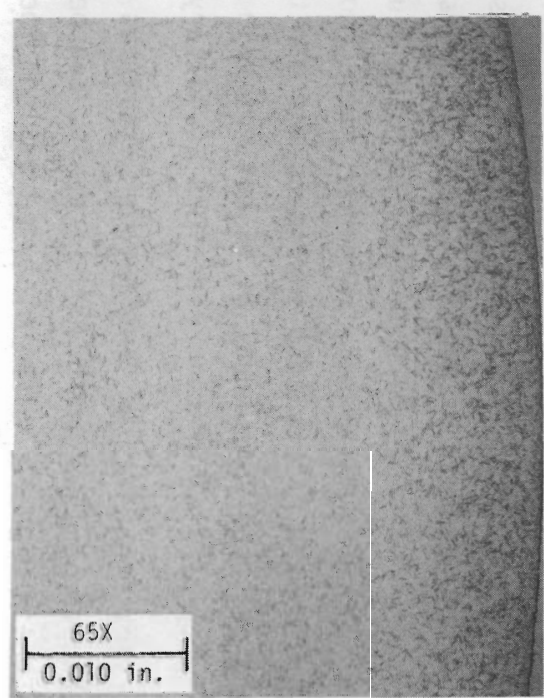

(f) STATIC TEST - DEMINERALIZED WATER,

FIGURE 18. Marcor Specimens Before and After Testing 
long-term leach resistance of the materials tested using the limited amount of data obtained in this study. Extrapolation of the measured leach rates to very long exposure times is difficult because in almost all of the tests the leach rates varied with time. In some of the dynamic leach tests the leach rates continued to decrease with time, and steady-state conditions were not attained. In the static tests the leach rates varied with time as expected, and in many of the tests the concentrations of dissolved species in the leach solutions did appear to reach saturation levels. In a static system, however, the extent of attack of the ceramic material will depend upon the volume of aqueous phase present and the S/V ratio for the system, and this parameter is unknown for a geologic repository. In addition, ceramographic examination of the test specimens was limited to samples that had been exposed for a single time period. Therefore, it is not possible to reliably estimate from this work how localized attack or alteration reactions would vary with exposure time.

Some very conservative "worst-case" estimates of the attack expected in a repository environment over a 1000-year exposure period can be made. In general, the materials tested exhibited their highest leach rates and maximum localized attack and alteration reactions in the dynamic tests at $250^{\circ} \mathrm{C}$. Therefore, extrapolation of the results from the short-term dynamic tests at $250^{\circ} \mathrm{C}$ should provide conservative estimates of long-term attack; this is especially true because the repository temperature would be much below $250^{\circ} \mathrm{C}$ for most of the 1000-year period. The estimates have been made and the results obtained are given in Table 16. The average corrosion of each material for a 1000-year exposure was estimated using the average leach rates given in Table 7 for the longest exposure time (43 or 45 days, depending on the material). The extent of localized attack or alteration reactions was estimated by linear extrapolation of the results given in Table 8 .

The very conservative "worst-case" estimates presented in Table 16 show that, except for $\mathrm{TiO}_{2}$ and graphite, all of the materials tested would suffer extensive corrosion when exposed to flowing demineralized water at $250^{\circ} \mathrm{C}$ for 1000 years. The estimated barrier thickness that would be required with most materials to provide the required level of isolation would probably be greater than could be fabricated with existing technology. In the case of $\mathrm{TiO}_{2}$ the canister thickness required, based on the estimate of average corrosion, would not be a problem; but the problem of cracking observed with the $\mathrm{TiO}_{2}$ specimens would have to be resolved before $\mathrm{TiO}_{2}$ could be considered for barrier use.

In a static system the long-term corrosion of the candidate materials should be much less than the values given in Table 16. Decreasing the temperature would also decrease the corrosion rate markedly, and at temperatures below $150^{\circ} \mathrm{C}$ localized attack and alteration reactions should not be a major problem with most of the candidate materials.

Detailed experimental evaluation of the corrosion behavior of ceramic materials under the conditions expected in a geologic repository would allow more reliable estimates of long-term corrosion to be made. These estimates would probably show that the thicknesses of ceramic barrier elements needed to provide the 1000-year protection would be considerably less than the values given in Table 16 . 
TABLE 16. Estimated Worst-Case Corrosion of Ceramic Material Exposed to Flowing Demineralized Water at $250^{\circ} \mathrm{C}$

\begin{tabular}{|c|c|c|}
\hline Material & Average(a) & $\begin{array}{l}\text { Localized Attack or } \\
\text { Alteration Reactions }(b)\end{array}$ \\
\hline $\mathrm{TiO}_{2}$ & 0.6 & $(c)$ \\
\hline Graphite & 1.5 & None \\
\hline $\mathrm{ZrO}_{2}$ & 7.5 & None \\
\hline $\mathrm{Al}_{2} \mathrm{O}_{3}(99.8 \%)$ & 13 & 21 \\
\hline $\mathrm{Al}_{2} \mathrm{O}_{3}(99 \%)$ & 18 & 42 \\
\hline $\mathrm{BaTiO}_{3}$ & 35 & 42 \\
\hline $\mathrm{CaZrO}_{3}$ & 40 & 290 \\
\hline $\mathrm{CaTiO}_{3}$ & 70 & 85 \\
\hline $\mathrm{CaTiSiO}_{5}$ & 80 & 160 \\
\hline $\begin{array}{l}\text { Vitreous Silica } \\
\left(\mathrm{SiO}_{2}\right)\end{array}$ & 85 & None \\
\hline $\mathrm{BaZrO}_{3}$ & 90 & 130 \\
\hline $\mathrm{ZrSiO}_{4}$ & 100 & 85 \\
\hline $\begin{array}{l}\text { Mullite } \\
\left(2 \mathrm{Al}_{2} \mathrm{O}_{3} \cdot \mathrm{SiO}_{2}\right)\end{array}$ & 170 & $>700$ \\
\hline Basalt & 350 & 450 \\
\hline Marcor & 500 & $>720$ \\
\hline Pyroceram 9617 & 1200 & $>1000$ \\
\hline
\end{tabular}

(a) Based on the average leach rates obtained in the screening tests--Table 7.

(b) Based on linear extrapolation of results obtained in the screening tests--Table 8.

(c) Specimen used in the screening test exhibited radial cracks to à considerable depth, but the results could not be extrapolated to long exposure times.

Although tests have not been carried out under identical conditions, it is interesting to compare reported corrosion rates for potential metallic barrier materials with the results obtained using ceramic materials. Studies were conducted at Sandia Laboratories (Braithwaite and Molecke 1978) to measure the corrosion of various metals in seawater and two brine solutions. The tests with deoxygenated brines were carried out by sealing test coupons in Pyrex or quartz ampules along with an aliquot of solution and heating them in an autoclave at $250^{\circ} \mathrm{C}$ for 2 to 8 weeks. The two brines used were 1) a brine similar in composition to the brine used in this study (primarily $\mathrm{NaCl}$ ) and 2) a high- $\mathrm{KCl}-\mathrm{NaCl}^{-} \mathrm{MgCl}_{2}$ brine (dissolved solids $2306 \mathrm{~g} / \mathrm{L}$ ). The results reported by Braithwaite and Molecke (1978) for the deoxygenated solutions are summarized in Table 17. A linear extrapolation of the data given in Table 17 shows that the more corrosion-resistant metals and alloys such as titanium, Zircaloy-2 and Ticode 12 (a titanium alloy) would suffer less attack in a 1000-year period than the best of the ceramic materials. 
TABLE 17. Corrosion Rates of Metals and Alloys in Deoxygenated Solutions $\left(250^{\circ} \mathrm{C}\right.$, $P=5 \mathrm{MPa}$ )

\begin{tabular}{|c|c|c|c|}
\hline Alloy & $\begin{array}{c}\text { Brine } A, \\
\mathrm{~cm} / \mathrm{yr}\end{array}$ & $\begin{array}{l}\text { Brine } B, \\
\mathrm{~cm} / \mathrm{yr}\end{array}$ & $\begin{array}{c}\text { Seawater, } \\
\mathrm{cm} / \mathrm{yr}\end{array}$ \\
\hline 1018 Mild Steel & 0.17 & 0.007 & 0.04 \\
\hline Corten A. Stee 1 & 0.09 & 0.005 & 0.02 \\
\hline 2-1/4 Cr-1 Mo Stee 1 & $0.10^{(a)}$ & $0.01^{(a)}$ & 0.02 \\
\hline Lead & 0.05 & 0.03 & 0.03 \\
\hline Copper & 0.007 & 0.005 & 0.005 \\
\hline Naval Brass & 0.1 & --- & 0.10 \\
\hline 90-10 Cupronickel & 0.014 & -- & 0.007 \\
\hline SS-304L & 0.0018 & 0.001 & 0.0006 \\
\hline SS-316L & 0.0015 & --- & 0.0005 \\
\hline SS-Nitronic 50 & 0.0008 & --- & 0.0003 \\
\hline SS-20Cb3 & 0.0007 & --- & 0.0005 \\
\hline SS-Ebrite $26-1$ & 0.0016 & --- & 0.0005 \\
\hline Mone 1-400 & 0.003 & --- & 0.01 \\
\hline Inco $10 y-825$ & 0.0006 & --- & 0.0004 \\
\hline Incone 1-600 & 0.0009 & 0.0007 & 0.0005 \\
\hline Incone 1-625 & 0.0005 & 0.0001 & $0.0012^{(b)}$ \\
\hline Haste1loy C-276 & 0.0007 & --- & 0.00015 \\
\hline Zircaloy-2 & 0.0001 & --- & --- \\
\hline Titanium C.P. & 0.0003 & --- & 0.0006 \\
\hline Ticode-12 & 0.00006 & -- & 0.0005 \\
\hline
\end{tabular}

(a) Crevice corrosion
(b) Pitting corrosion

If one allows for the differences in the conditions under which the tests were conducted, however, it is impossible to say if the best metallic materials are more corrosion-resistant than are graphite and the best of the ceramic materials. In order to obtain meaningful comparisons of the corrosion resistance of the two groups of materials, the corrosion tests would have to be carried out under similar conditions. 


\subsection{CONCLUSIONS AND RECOMMENDATIONS}

Based on the results obtained in the various leach tests there are several general conclusions that can be reached regarding the leach resistance of the candidate materials evaluated in this study.

- On an overall basis, graphite exhibited the greatest leach resistance of the materals tested. Attack of the graphite appeared to depend primarily upon the dissolved oxygen in the leach solution.

- All of the ceramic materials tested suffered extensive attack in one or more of the leach tests. Overall, the two grades of alumina appeared to be the most leach-resistant of the ceramic materials tested. Titanium dioxide and $\mathrm{ZrO}_{2}$ were the best of the remaining materials.

- Localized attack or alteration reactions presented a serious problem at temperatures of $150^{\circ} \mathrm{C}$ and above with most of the ceramic materials. Only $\mathrm{ZrO}_{2}$ and $\mathrm{TiO}_{2}$ did not appear to suffer from localized attack or alteration reactions, although some of the $\mathrm{TiO}_{2}$ specimens exhibited cracking that may have resulted from fabrication problems rather than attack by the leach solutions.

- The leach rates increased with temperatures as expected. Direct comparisons are difficult because the leach rates varied with time. But in some cases the increase in leach rates with temperature were not as great as one might anticipate. Figure 19, for example, shows the effect of temperature on the average leach rates of several materials exposed to flowing demineralized water at $90^{\circ}$ and $250^{\circ} \mathrm{C}$. The values given in the figure are the average leach rates for the longest exposure time used in the tests ( 35 days at $90^{\circ} \mathrm{C}$ and 43 or 45 days at $250^{\circ} \mathrm{C}$ ). For some materials, such as mullite and Pyroceram 9617, the leach rate increased 500 to 1000 times as the temperature increased from $90^{\circ}$ to $250^{\circ} \mathrm{C}$, whereas the leach rate of $\mathrm{TiO}_{2}$ approximately doubled over the same temperature range. In the static tests, where the leach rates were limited by saturation effects, the increases in the average leach rates for the various materials were generally less than an order of magnitude over the temperature range of $150^{\circ}$ to $250^{\circ} \mathrm{C}$.

- In the static tests at $150^{\circ}$ and $250^{\circ} \mathrm{C}$ the leach rates were generally highest in the demineralized water and lowest in the brine solution, with the Hanford ground water giving intermediate results.

- In most of the tests the leach rates varied with time, although in some of the dynamic tests it appeared that steady-state conditions were attained. In most of the static tests it was apparent that saturation of dissolved species in the leach solutions served to 1 imit dissolution of the ceranic materials.

The conclusions stated above are based on an overall evaluation of the test results, and may not be valid for a specific repository environment where conditions could be quite different from those used in the leach tests. 


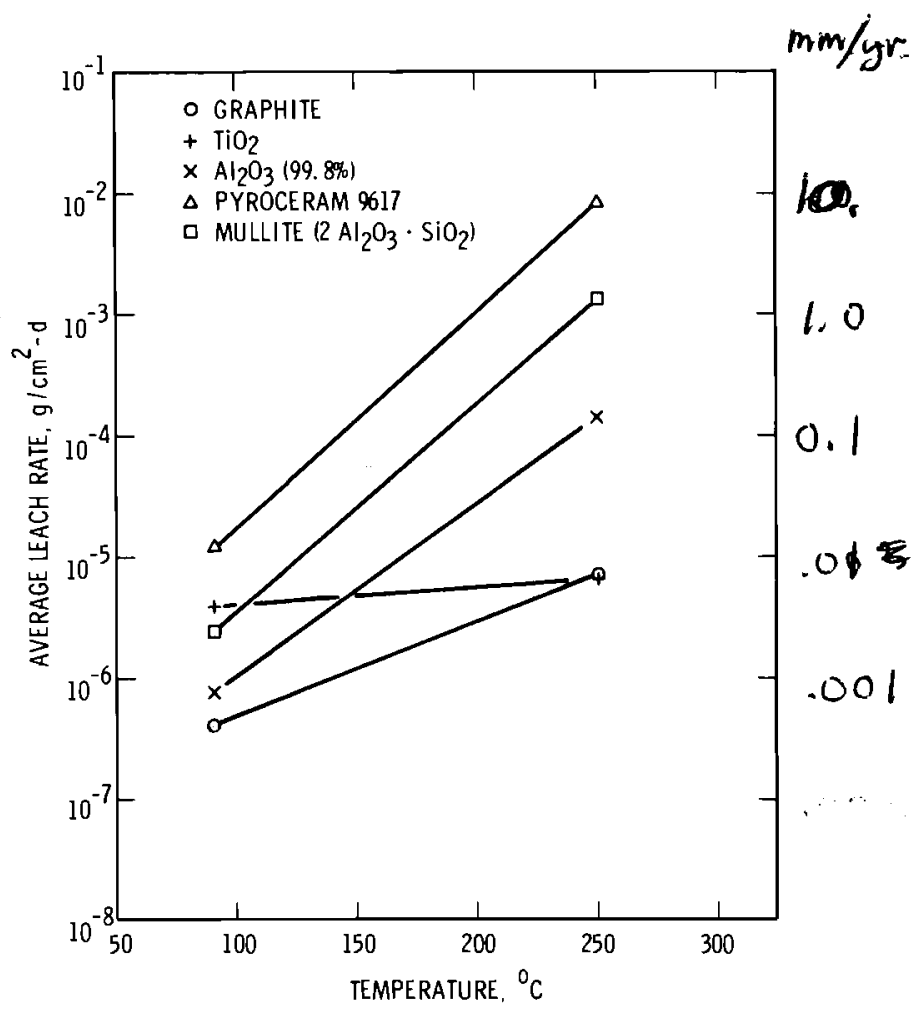

FIGURE 19. The Effect of Temperature on the Leaching of Various Materials

The results obtained in this study are not adequate to demonstrate the feasibility of ceramic barrier elements. Additional research and development will be required to demonstrate that one or more of the materials tested can be used successfully in engineered-barrier systems. The results of this work do show, however, which of the materials offers the best chance of developing acceptable barrier elements. Based on the experimental results obtained, and considering other factors such as cost, availability, fabricability, mechanical and physical properties, graphite appears to be the primary choice for the barrier application, with alumina as the second choice. If the problem of cracking can be overcome, $\mathrm{TiO}_{2}$ would also be a likely choice. Zirconium oxide appears to be quite resistant to the leach solutions, but does not appear to warrant further consideration as a barrier because of its thermal-shock sensitivity and fabrication difficulties. None of the other ceramic materials tested appear to warrant further consideration for the barrier application.

If the decision is made to continue with the development of nonmetallic barrier elements, its is recommended that graphite, alumina and possibly $\mathrm{TiO}_{2}$ be the materials considered for detailed evaluation. 


\subsection{REFERENCES}

Ames, L. L. 1978. Low Temperature Radionuclide Kd Values for Basalt, Heulandite and Nontron ite. PNL-2817, Pac if ic Northwest Laboratory, Richland, Washington.

Braithwaite, J. W. and M. A. Molecke. 1978. High-Level Waste Canister Corrosion Studies Pertinent to Geologic Isolation. SAND 78-2111, Sandia Laboratories Albuquerque, New Mexico. (Presented at the Conference on High-Level Radioactive Solid Waste Forms, sponsored by the NRC, Denver, Colorado, December 19-21, 1978).

Gray, W. J. 1980. Radioactive Waste Management. 1:105.

Lynch, J. F., C. G. Ruderer, W. H. Duckworth (eds.). 1966. Engineering Properties of Selected Ceramic Materials. Compiled by Battelle Memorial Institute, Columbus, Ohio and published by the American Ceramic Society, Columbus, Ohio. 



\subsection{ACKNOWLEDGMENTS}

Most of the experimental studies were perfored by R. J. Elovich. Autoclave testing was provided by R. A. Clark. Ceramographic examination of the test specimens was provided by R. H. Beauchamp and F. T. Hara provided the analys is of the leach solutions.

R. E. Westerman was the Task Leader. The report was edited by M. H. Henry and reviewed by D. E. Larson, H. H. Van Tuyl and R. E. Westerman. 


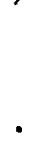


APPENDIX

DESCRIPTION OF MATERIALS STUDIED 


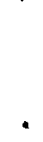


What follows are general descriptions and lists of characteristics for each of the materials studied.

$\mathrm{Al}_{2} \underline{0}_{3}(99 \%)$

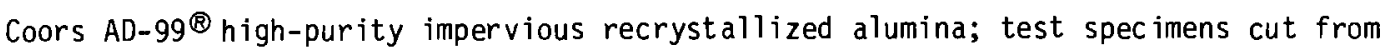
1/4-in.-dia rod obtained from the manufacturer.

Porosity--gas-tight

Water adsorption--none

Micrographs--show some unconnected internal voids

Density-->90\% of theoretical density

Surface area (BET) of test specimen-- $<10 \mathrm{~cm}^{2} / \mathrm{g}$

$\mathrm{Al}_{2}{ }_{2}{ }_{3}(99.8 \%)$

McDanel high-purity impervious alumina; ${ }^{\circledR}$ test specimens cut from 1/4-in.-dia rod obtained from manuf acturer.

Porosity--gas-tight

Water adsorption--none

Micrographs--show some unconnected internal voids

Density-->92\% of theoretical density

Surface area (BET) of test. specimens $--<10 \mathrm{~cm}^{2} / \mathrm{g}$

\section{MULLITE $\left(2 \mathrm{Al} 2_{2}{ }_{-3} \cdot \mathrm{SiO}_{2}\right)$}

Coors impervious Mullite ${ }^{\oplus}$ (Sillmanite); test pieces cut from 1/4-in.-dia rod obtained from manuf acturer.

Porosity--gas-tight

Water adsorption--none

Micrographs--show some unconnected internal voids

Density-->90\% of theoretical density

Surface area (BET) of test specimens $--<10 \mathrm{~cm}^{2} / \mathrm{g}$

\footnotetext{
B Coors Porcelain Co., Golden, Colorado

$\otimes_{M C D}$ anel Refractory Porcelain Co., Beaver Falls, Pennsylvania
} 
Test specimens cut from a sample of basalt taken from Flow $E$ of the Sentine 1 Bluffs - Grande Ronde Formation.

Micrographs--show some slight internal porosity

Average density--3.17 $\mathrm{g} / \mathrm{cm}^{3}$

Surface area (BET) of test specimens-- $100 \mathrm{~cm}^{2} / \mathrm{g}$

$\underline{\mathrm{BaTi0}}_{3}$

$\mathrm{BaTiO}_{3}$ powder obtained from Cerac, Inc.; (a) test specimens were prepared by cold-pressing the $\mathrm{BaTiO}_{3}$ powder into pellets at 50 tons/in. ${ }^{2}$ and sintering the pellets at $21200^{\circ} \mathrm{C}$ for $90 \mathrm{~h}$.

Purity $98 \%$ analysis: $A 1=0.01-0.1 \%$

$C_{a}=0.01-0.1$

$\mathrm{Co}_{0}=0.001$

$\mathrm{Cu}=0.005-0.005$

$\mathrm{Fe}=0.005-0.05$

$\mathrm{Mg}=0.01-0.1$

$\mathrm{Si}=0.01-0.1$

$V=0.001-0.01$

Particle size: -325 mesh +5 microns

Micrographs of the pellets--show extensive connecting internal porosity

Average pellet density-- $89 \%$ of theoretical density

Surface area (BET) of pellets-- $320 \mathrm{~cm}^{2} / \mathrm{g}$

\section{$\underline{B a Z r O}_{3}$}

$\mathrm{BaZrO}_{3}$ powder obtained from Cerac, Inc.; test specimens were prepared by cold-pressing $\mathrm{BaZr}_{2} \mathrm{O}_{3}$ powder into pellets at 50 tons $/$ in. $^{2}$ and sintering the pellets at $\sim 1200^{\circ} \mathrm{C}$ for $90 \mathrm{~h}$.

$$
\begin{aligned}
\text { Purity 99\% analysis: } & \mathrm{Al}=0.03-0.3 \% \\
\mathrm{Ca} & =0.01-0.1 \\
\mathrm{Cu} & =0.0001 \\
\mathrm{Fe} & =0.01-0.1 \\
\mathrm{Mg} & =0.01-0.1 \\
\mathrm{Si} & =0.1-1.0 \\
\mathrm{Ti} & =0.03-0.3
\end{aligned}
$$

(a) Cerac, Inc., Milwaukee, Wiscons in 
Particle Size: -325 mesh

Micrographs of the pellets--show extensive internal porosity

Average pellet density-- $292 \%$ of theoretical density

Surface area (BET) of pellets $--\sim 700 \mathrm{~cm}^{2} / \mathrm{g}$

\section{$\underline{\mathrm{CaTiO}}_{3}$}

$\mathrm{CaTiO}_{3}$ powder obtained from Cerac, Inc.; test specimens were prepared by hot-pressing the $\mathrm{CaTiO}_{3}$ powder into pellets at $1600^{\circ} \mathrm{C}$ and 5200 psi using graphite dies, then heating the pellets in air at $\sim 1200^{\circ} \mathrm{C}$ for $24 \mathrm{~h}$.

$$
\text { Purity 99\% analysis: } \begin{aligned}
A 1 & =0.005-0.05 \% \\
\text { Co } & =0.01-0.01 \\
C U & =0.001 \\
\text { Fe } & =0.001 \\
\text { Mo } & =0.001 \\
& \text { Pb }=0.005-0.005 \\
S_{i} & =0.001-0.011 \\
S_{r} & =0.001-0.01 \\
& \mathrm{Zr}=0.001-0.01
\end{aligned}
$$

Particle Size: -325 mesh

Micrographs of the pellets--showed some internal porosity

Average pellet density-- $294 \%$ of theoretical density

Surface area (BET) of pellets-- $4450 \mathrm{~cm}^{2} / \mathrm{g}$

\section{$\underline{\mathrm{CaZr}}_{3}$}

$\mathrm{CaZrO}_{3}$ powder obtained from Cerac, Inc.; test specimens prepared by cold-pressing $\mathrm{CaZrO}_{3}$ into pellets at 50 tons $/ \mathrm{in}^{2}$ and sintering the pellets at $21200^{\circ} \mathrm{C}$ for $90 \mathrm{~h}$.

$$
\text { Purity 99\% analysis: } \begin{aligned}
\mathrm{A} 1 & =0.01-0.1 \% \\
\mathrm{Ba} & =0.003-0.03 \\
\mathrm{Cu} & =0.001-0.01 \\
\mathrm{Fe} & =0.03-0.3 \\
\mathrm{Mg} & =0.001-0.01 \\
\mathrm{Si} & =0.15 \\
\mathrm{Ti} & =0.03-0.3
\end{aligned}
$$

Particie size: -325 mesh

Micrographs of the pellets--show some unconnected internal voids

Average pellet density-- $91 \%$ of theoretical density

Surface area (BET) of pellets--too low to measure $\left(<10 \mathrm{~cm}^{2} / \mathrm{g}\right)$. 
$\underline{\mathrm{CaTiSi0}}_{5}$

$\mathrm{CaTiSiO}{ }_{5}$ was prepared by reacting $\mathrm{CaTiO}_{3}$ and $\mathrm{SiO}_{2}$ powders at high temperatures.

The $\mathrm{CaTiO}_{3}$ and $\mathrm{SiO}_{2}$ powders were obtained from Cerac, Inc. The purity and analysis of the $\mathrm{CaTiO}_{3}$ powder is given in a previous section. Purity of the $\mathrm{SiO}_{2}$ was $99.9 \%$ and the particle size was -325 mesh. Analysis of the material was as follows:

$$
\begin{aligned}
A 1 & =0.003-0.01 \% \\
B & =0.001-0.01 \\
C a & =0.01-0.1 \\
\mathrm{Fe} & =0.005-0.05 \\
\mathrm{Mg} & =0.005-0.05 \\
\mathrm{Mn} & =0.001 \\
\mathrm{~Pb} & =0.001 \\
\mathrm{Ti} & =0.01-0.1 .
\end{aligned}
$$

Stoichiometric quantities of the powders were wet-milled and then vacuum-dried. The mixed powders were fused in a platinum crucible at $1600^{\circ} \mathrm{C}$. After cooling, the product was crushed to a fine powder. X-ray diffraction analys is of the powder showed it to be $\mathrm{CaTiSiO}_{5}$ with no indication of other phases being present.

The test specimens were prepared by cold-pressing the powder into pellets at 50 tons $/$ in. ${ }^{2}$ and sintering the pellets at $\sim 1200^{\circ} \mathrm{C}$ for $90 \mathrm{~h}$.

Micrographs of the pellets--showed some connecting internal porosity. Average pellet density-- $90 \%$ of theoretical density Average surface area (BET) of pellets-- $2900 \mathrm{~cm}^{2} / \mathrm{g}$.

\section{VITREOUS SILICA $\left(\mathrm{SiO}_{2}\right)$}

Amersil Infrasil vitreous silica glass; ${ }^{\circledR}$ test specimens cut from 1/4-in.-dia rod obtained from the manufacturer.

Porosity--gas-tight

Water adsorption--none

Micrographs--show no indication of internal porosity Density--2.20 $\mathrm{g} / \mathrm{cm}^{3}$

Surface area (BET) of test specimens-- $<10 \mathrm{~cm}^{2} / \mathrm{g}$

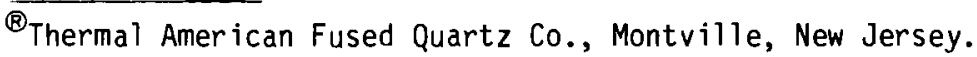


$\underline{\mathrm{TiO}}_{2}$

$\mathrm{TiO}_{2}$ powder obtained from Cerac, Inc.; test specimens were prepared by hot-pressing the $\mathrm{TiO}_{2}$ powder into pellets at $1600^{\circ} \mathrm{C}$ and 5200 psi using graphite dies, then firing the pellets in air at $\sim 1200^{\circ} \mathrm{C}$ for $24 \mathrm{~h}$.

$$
\begin{aligned}
& \text { Purity } 99.9 \% \text { analysis: } \quad A I=0.01-0.1 \% \\
& \mathrm{Ca}=0.01-0.1 \\
& C_{0}=0.001-0.01 \\
& \mathrm{Cr}=0.01-0.1 \\
& \mathrm{Cu}=0.001 \\
& \mathrm{Fe}=0.05-0.1 \\
& \mathrm{Mg}=0.01-0.1 \\
& \mathrm{Ni}=0.001 \\
& \mathrm{Sb}=0.001-0.01 \\
& S i=0.01-0.1 \\
& S n=0.001 \\
& v=0.001 \\
& \mathrm{Zr}=0.05-0.5
\end{aligned}
$$

Particle Size: -325 mesh +10 microns

Micrographs--show extensive internal porosity

Average pellet density--93\% of theoretical density

Surface area (BET)-- $220 \mathrm{~cm}^{2} / \mathrm{g}$

$\underline{\operatorname{Zr}}_{2}$

Degussit $\mathrm{Zr}-23 \mathrm{CaO}$-stabilized $\mathrm{ZrO}_{2}{ }^{(\mathrm{a})}\left(>98 \% \mathrm{ZrO}_{2}\right)$; test specimens were cut from impervious tubing obtained from the manuf acturer.

Porosity--gas-tight

Micrographs--show extensive unconnected internal porosity

Density--91\% of theoretical density

Surface area (BET) of test specimens- $\sim 20 \mathrm{~cm}^{2} / \mathrm{g}$

(a) Supplied by Alpha Products, Donvers, Maine. 
$\mathrm{ZrSiO}_{4}$ powder obtained from Cerac, Inc.; test specimens were prepared by hot-pressing $\mathrm{ZrSiO}_{4}$ powder into pellets at $\sim 1600^{\circ} \mathrm{C}$ and 5200 psi using graphite dies, then firing the pellets in air at $21200^{\circ} \mathrm{C}$ for $24 \mathrm{~h}$.

$$
\begin{aligned}
\text { Purity 99\% Analysis: } & \text { Al } 0.03-0.3 \% \\
& \text { Fe } 0.01-0.1 \\
& \text { Mg } 0.001 \\
& \text { Ti } 0.03-0.3
\end{aligned}
$$

Particle Size: -200 mesh +10 microns

Micrographs of the pellets--show extensive interconnected internal porosity

Average pellet density--85\% of theoretical density

Surface area (BET) of test specimens-- $21120 \mathrm{~cm}^{2} / \mathrm{g}$

\section{GRAPHITE (TOYO)}

Toyot anso Graphite IG-11 ${ }^{\circledR}$ for HTGR; test specimens cut from a block of graphite obtained from the manuf acturer.

Density--1.80 $\mathrm{g} / \mathrm{cm}^{3}$

Micrographs--showed extensive interconnected external porosity

Surface area (BET) of test specimens-- $10,000 \mathrm{~cm}^{2} / 9$

\section{PYROCERAM 9617}

Pyroceram $9617 \mathrm{glass-ceramic} \mathrm{from} \mathrm{Corning} \mathrm{Glass} \mathrm{Works;} \mathrm{test} \mathrm{specimens} \mathrm{cut} \mathrm{from} \mathrm{a} \mathrm{block} \mathrm{of}$ material supplied by the manufacturer.

Porosity--gas-tight

Micrographs--show very little internal porosity

Density--2.52 $\mathrm{g} / \mathrm{cm}^{3}$

Surface area (BET) of test specimens $--<10 \mathrm{~cm}^{2} / \mathrm{g}$

\section{MARCOR MACHINABLE GLASS-CERAMIC}

Marcor Code 9658 machinable glass-ceramic from Corning Glass Works; test specimens cut from 8-mm-dia rod obtained from the manufacturer.

Water adsorption--none

Micrographs--show only very slight internal porosity

Density--2.52 $\mathrm{g} / \mathrm{cm}^{3}$

Surface area (BET) of test specimens $--<10 \mathrm{~cm}^{2} / \mathrm{g}$.

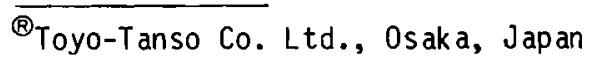




\section{DISTRIBUTION}

No. of

Copies

\section{UNITED STATES}

A. A. Churm

DOE Chicago Patent Division

9800 South Cass Avenue

Argonne, IL 60439

R. E. Cunningham

Deputy Director for Fuels and

Materials

Nuclear Regulatory Commission

Silver Springs, MD 20910

T. C. Chee

DOE Office of Nuclear Waste Management Washington, DC 20545

C. R. Cooley

DOE Office of Nuclear Waste Management Washington, DC 20545

She ldon Meyers

DOE Office of Nuclear Waste Management Washington, DC 20545

R. G. Romatowski

DOE Office of Nuclear Waste Management Washington, DC 20545

C. A. Heath

DOE Office of Nuclear Waste Management Washington, DC 20545

G. Oertel

DOE Office of Nuclear Waste Management Washington, DC 20545

A. F. Perge

DOE Office of Nuclear Waste Management Washington, DC 20545

R. D. Walton

DOE Office of Nuclear Waste Management Washington, DC 20545

J. Neff, Program Manager

Department of Energy

Columbus Progran Office

505 King Avenue

Columbus, $\mathrm{OH} \quad 43201$

John $V$ an Cleve

DOE Oak Ridge Operations office

P.0. Box $X$

Oak Ridge, TN 37830
No. of

Copies

J. B. Whitsett

DOE Idaho Operations office

550 2nd Street

Idaho Falls, ID 83401

E. S. Goldberg

DOE Savannah River Operations Office P. 0. Box A

Aiken, SC 29801

27 DOE Technical Information Center

J. R. Berreth

Allied Chemical Corporation

550 2nd Street

Idaho Falls, ID 83401

Allied Chemical Corporation

(File Copy)

550 2nd Street

Idaho Falls, ID 8340 l

A. Williams

Allied-General Nuclear Service

P.0. Box 847

Barnwe 11, SC 29812

J. L. Jardine

Argonne National Laboratory

9700 South Cass Avenue

Argonne, IL 60439

M. M. Steindler/L. E. Trevorrow

Argonne National Laboratory

9700 South Cass Avenue

Argonne, IL 60439

Wayne Carbiener

Office of Nuclear Waste Isolation

Battelle Memorial Institute

505 King Ave.

Columbus, $\mathrm{OH} 43201$

J. Kircher

Office of Nuclear Waste Isolation

Battelle Memorial Institute

505 King Ave.

Columbus, $\mathrm{OH} \quad 43201$

Beverly Rawles

Office of Nuclear Waste Isolation Battelle Memorial Institute

505 King Ave.

Columbus, $\mathrm{OH} 43201$ 
No. of

Copies

J. W. Voss

Office of Nuclear Waste I solation

Battelle Memorial Institute

505 King Ave.

Columbus, $\mathrm{OH} 43201$

Brookhaven National Laboratory

Reference Section

Information Division

Upton, NY 11973

M. D. Boersma

E. I. duPont DeNemours and Company

Savannah River Laboratory

Aiken, SC 29801

J. L. Crandall

E. I. duPont DeNemours and Company

Savannah River Laboratory

Aiken, SC 29801

H. L. Hull

E. I. duPont DeNemours and Company

Savannah River Laboratory

Aiken, SC 29801

D. E. Gordon

E. I. DuPont DeNemours and Company

Savannah River Laboratory

Aiken, SC 29801

R. G. Garvin

E. I. duPont DeNemours and Company

Savannah River Laboratory

Aiken, SC 29801

D. L. McIntosh

E. I. duPont DeNemours and Company Savannah River Laboratory

Aiken, SC 29801

J. A. Kelley

E. I. duPont DeNemours and Company

Savannah River Laboratory

Aiken, SC 29801

S. Mirshak

E. I. duPont DeNemours and Company Savannah River Laboratory

Aiken, SC 29801

A. S. Jennings

E. I. duPont DeNemours and Company

Savannah River Laboratory

Aiken, SC 29801

H. Henning

Electric Power Research Institute

3412 Hillview Avenue

P.0. Box 10412

Palo Alto, CA 94301
No. of

Copies

Environmental Protection Agency

Technology Assessment Division

(AW-559)

Office of Radiation Programs

Washington, DC 20460

R. G. Barnes

General Electric Company

175 Curtner Avenue (M/C 858)

San Jose, CA 95125

Los Alamos Scientific Laboratory (DOE)

P.0. Box 1663

Los Alamos, NM 87544

J. P. Duckworth

Plant Manager

Nuclear Fuel Services, Inc.

P.0. Box 124

West Valley, NY 14171

J. G. Cline, General Manager

NYS ERDA

Agency Building \#2

Empire State Plaza

Albany, NY 12233

Oak Ridge National Laboratory (DOE)

Central Research Library

Document Reference Section

P.0. Box X

Oak Ridge, TN 37830

E. H. Kobisk

Solid State Division

Oak Ridge National Laboratory

Oak Ridge, TN 37830

R. Roy

Pennsylvania State University

Materials Research Laboratory

University Park, PA 16802

W. Weart

Sand ia Laboratories

Albuquerque, NM 87107

J. 0. Blomeke

Union Carbide Corporation (ORNL)

Chemical Technology Division

P.0. Box $Y$

Oak Ridge, TN 37830

R. E. Blanco

Union Carbide Corporation (ORNL)

Chemical Technology Division

P.O. Box Y

0ak Ridge, TN 37830 
No. of

Copies

D. E. Ferguson

Union Carbide Corporation (ORNL)

Chemical Technology Division

P.0. Box $Y$

Oak Ridge, TN 37830

H. W. Godbee

Union Carbide Corporation (ORNL)

Chemical Technology Division

P.0. Box Y

Oak Ridge, TN 37830

4 DOE Richland Operations office
P. A. Craig
R. B. Gor anson
H. E. Ransom
M. J. Zamorski

8 Rockwe 11 Hanford Operations
L. Brown
R. A. Deju
M. J. Kupfer
E. L. Moore
G. Reep
M. J. Smith
D. D. Wodrich
File copy

Exxon Nuclear Company

S. J. Beard

2 West inghouse Hanford Comp any
A. G. Blasewitz
D. A. Cantley

\section{Pacific Northwest Laboratory}
S. M. Barnes
W. J. Bjorklund
H. T. Blair
W. F. Bonner
R. A. Brouns
J. L. Buelt
L. A. Chick
R. D. Dierks
R. L. Dillon
H. T. Fullam (20)
M. S. Hanson
A. J. Haverfield
M. H. Henry (3)
L. K. Holton
J. H. Jarrett
A. B. Johnson

No. of

Copies

Pacific Northwest Laboratory

D. E. Knowlton

C. A. Knox

M. R. Kreiter

W. L. Kuhn

D. E. Larson (2)

J. L. McElroy (3)

G. B. Mellinger

J. E. Mende 1

M. D. Merz

F. A. Miller

T. Nelson

R. E. Nightingale

R. D. Nelson

D. E. Olesen

K. H. Oma

C. R. Palmer

P. J. Pelto

A. M. Platt

D. L. Prezbindowski (2)

W. A. Ross

J. M. Rusin

R. J. Serne

D. H. Siemens

S. C. Slate

C. L. Timmerman

R. T. Treat

R. D. Westerman (5)

Technical Information (5)

Publishing Coordination EI(2) 